\title{
RATIONAL SUBSETS IN HNN-EXTENSIONS AND AMALGAMATED PRODUCTS
}

\author{
Markus Lohrey \\ Universität Stuttgart, FMI, Germany \\ lohrey@informatik.uni-stuttgart.de \\ Géraud Sénizergues \\ LABRI, Université Bordeaux I, France \\ ges@labri.fr
}

\begin{abstract}
Several transfer results for rational subsets and finitely generated subgroups of HNNextensions $G=\left\langle H, t ; t^{-1} a t=\varphi(a)(a \in A)\right\rangle$ and amalgamated free products $G=H *_{A} J$ such that the associated subgroup $A$ is finite. These transfer results allow to transfer decidability properties or structural properties from the subgroup $H$ (resp. the subgroups $H$ and $J$ ) to the group $G$.
\end{abstract}

\section{Introduction}

This paper is the first paper in a series of papers dealing with algorithmic problems for equations over HNN-extensions [HNN49] and amalgamated free products of groups. These two operations are of fundamental importance in combinatorial group theory [LS77]. Recall that an amalgamated free product

$$
G=\left\langle H_{1} * H_{2}, a=\varphi(a)(a \in A)\right\rangle
$$

of two groups $H_{1}$ and $H_{2}$ with subgroups $A \leq H_{1}, B \leq H_{2}$ and an isomorphism $\varphi: A \rightarrow B$, results from the free product $H_{1} * H_{2}$ by identifying every element $a \in A_{1}$ with $\varphi(a) \in A_{2}$. We also say that $A$ and $B$ in (1) are the identified subgroups. An HNN extension

$$
G=\left\langle H, t ; t^{-1} a t=\varphi(a)(a \in A)\right\rangle
$$

of a group $H$, where $A$ and $B$ are isomorphic subgroups of $H$ and $\varphi: A \rightarrow B$ is an isomorphism, results from adding to $H$ a new generator $t$ such that the conjugation of the subgroup $A \leq H$ by $t$ realizes the isomorphism $\varphi$. We also say that $A$ and $B$ in (2) are the associated subgroups.

One of the first important applications of HNN-extensions was a more transparent proof of the celebrated result of Novikov and Boone on the existence of a finitely presented group with an undecidable word problem, see e.g. [LS77]. Such a group can be constructed by a series of HNN-extensions starting from a free group. This shows that arbitrary HNN-extensions do not have good algorithmic properties. In 
this paper, we restrict to HNN-extensions (resp. amalgamated free products), with finite associated (resp. identified) subgroups. This is an important special case. For instance, a famous result of Stallings states that a group $G$ has more than one end if and only if it is either an HNN-extension with finite associated subgroups or an amalgamated free product with finite identified subgroups [Sta71]. Moreover, a group is virtually-free (i.e., has a free subgroup of finite index) if and only if it can be built up from finite groups using amalgamated free products with finite identified subgroups and HNN-extensions with finite associated subgroups [DD90]. Finally, many important structural and algorithmic properties of groups are preserved by HNN-extensions (resp. amalgamated free products) with finite associated (resp. identified) subgroups, see e.g. [AG73,Bez98,KSS06,KS70,KS71,KWM05,MR04].

In this paper, we will prove several such preservation results for rational subsets and finitely generated subgroups of HNN-extensions and amalgamated free products of groups. Recall that the set of rational subsets of a monoid $\mathbb{M}$ is the smallest class of subsets of $\mathbb{M}$ which contains all finite subsets of $\mathbb{M}$ and which is closed under union, product and the Kleene star (which associates with a subset $U \subseteq \mathbb{M}$ the submonoid of $\mathbb{M}$ generated by $U$ ). By Kleene's theorem, the rational subsets of a free monoid are a Boolean algebra. This result also holds for free groups [Ben69] and even virtually-free groups [Sén96]. Further results on rational subsets of groups can be found in [Gru90,KSS06,Ned00].

Some of our results are already known, others appear to be new. Apart from proving these new results, the purpose of our investigations is two-fold: first, we prepare the background for our further considerations on equations over HNNextensions and amalgamated free products [LS06a,LS06b]. Second, we present a unified automata-theoretic framework, which is partly inspired by [Reu85,Sak87, Sak92], for reasoning about rational subsets of HNN-extensions and amalgamated free products. This framework allows us to deduce transfer theorems in a unified way. Moreover, our approach allows us to prove results about rational subsets even for HNN-extensions and amalgamated free products of monoids, in case the associated parts $A$ and $B$ in (1) and (2) are groups. Let us now describe in more detail the content of this paper.

Section 2 contains necessary preliminaries concerning monoids, groups, and rational sets. Section 3 contains basic definitions and facts for HNN-extensions. In Section 4.1, we introduce finite $t$-automata, which are a specific automaton model in order to recognize subsets of an HNN-extension $G=\left\langle H, t ; t^{-1} a t=\varphi(a)(a \in A)\right\rangle$, where $H$ is a monoid and $A$ and $B=\varphi(A)$ are finite groups. The transitions of such an automaton are either labelled with $t, t^{-1}$, or sets from some subset $\mathcal{F} \subseteq \mathcal{P}(H)$ (which has to contain every singleton subset consisting of an element from $A \cup B$ ). For our specific applications concerning rational subsets (resp. finitely generated subgroups) of the HNN-extension $G, \mathcal{F}$ is the set of rational subsets of $H$ (resp. the set of left cosets of finitely generated subgroups of $H$ ). In Section 4.2-4.3 we prove several basic facts about finite $t$-automata and in Section 4.4 we prove two 
fundamental normalization results for finite $t$-automata (Propositions 22, 28 and 33 ). Roughly speaking, these normalization results state that we can always enforce some nice properties for finite $t$-automata. Based on these results we can show in Section 4.5 Theorem 37, which is our main result for rational subsets of HNNextensions. It says that the following four properties can be transfered from the monoid $H$ to the HNN-extension $G=\left\langle H, t ; t^{-1} a t=\varphi(a)(a \in A)\right\rangle$ (with $A$ and $B$ finite groups):

(a) The set of rational subsets is closed under intersection.

(b) The set of rational subsets is closed under complement (i.e., it is a Boolean algebra).

(c) The membership problem for rational subsets is decidable.

(d) The emptiness problem for boolean combinations of rational subsets is decidable.

Statement (c) is covered by [KSS06, Theorem 5.1] in the case that $H$ (and hence $G$ ) is a group, whereas (a), (b), and (d) are new to the knowledge of the authors. It should be remarked that properties (a) and (b) (resp. (c) and (d)) are independent in the following sense: Clearly, closure under complement for the rational subsets implies closure under intersection. On the other hand, in Appendix B, we construct a finitely generated monoid for which the rational subsets are closed under intersection but are not closed under complement (we don't know, whether a finitely generated group with this property exists as well). Similarly, decidability of the emptiness problem for boolean combinations of rational subsets implies decidability of the membership problem for rational subsets. On the other hand, for the group $F \times \mathbb{Z}$ (where $F$ is the free group of rank 2), the membership problem for rational subsets is decidable [KSS06], whereas the inclusion problem for rational subsets (which is a special instance of the emptiness problem for boolean combinations of rational subsets) is undecidable [AH89].

Let us mention that more general notions of HNN-extensions for monoids were defined in the literature (see for instance [How63]) where $A$ and $B$ in (2) are not necessarily groups. It is not clear whether our results hold for these HNN-extensions as well.

In Section 4.6 we deal with finitely generated subgroups of HNN-extensions. Here we assume that $H$ (and hence $G$ ) in (2) is a group. Our main result is Theorem 43, which states that the following three properties can be transfered from a group $H$ to the HNN-extension $G=\left\langle H, t ; t^{-1} a t=\varphi(a)(a \in A)\right\rangle$ (with $A$ and $B$ finite):

(a) Decidability of the generalized word problem (i.e., the question whether a given group element belongs to a given finitely generated subgroup).

(b) The Howson property.

(c) The LERF property. 
Recall that a group $G$ has the Howson property if the intersection of two finitely generated subgroups is again finitely generated. The group $G$ has the LERF property [Hal49] if for every finitely generated subgroup $H<G$ and every $g \in G \backslash H$ there exists a subgroup $Z$ of finite index in $G$ with $H \leq Z<G$ and $g \in G \backslash Z$. Statement (a) is covered by [KWM05, Corollary 5.15], statement (b) was shown in [Bez98,KS71], and statement (c) was shown in [MR04].

Finally, in Section 6 we briefly sketch the proof of analogous transfer results for amalgamated free products (Theorem 51 and 53). Since the treatment of amalgamated free products exactly follows our treatment of HNN-extensions, we will just state the necessary adaptations but omit most of the proof details. For the special case of an amalgamated free product of two finite groups, algorithmic problems for finitely generated subgroups were recently studied in [ME07b,ME07a] by extending Stalling's folding technique [Sta83].

\section{Preliminaries}

We recall in this section all needed definitions and classical results concerning groups, monoids, finite automata, and rational subsets of a monoid. We also recall some classical algorithmic problems about rational subsets of finitely generated monoids.

We assume that the reader has some familiarity with monoid presentations and group theory. For a monoid homomorphism $\pi: \mathbb{M}_{1} \rightarrow \mathbb{M}_{2}$ we denote with $\operatorname{Ker}(\pi)=$ $\{(x, y) \mid \pi(x)=\pi(y)\}$ the kernel of $\pi$, it is a monoid congruence on $\mathbb{M}_{1}$. The neutral element 1 of a monoid $\mathbb{M}$ will be identified with the submonoid $\{1\}$ in the rest of this paper.

The powerset of a set $S$ is denoted by $\mathcal{P}(S)$. For an equivalence relation $\equiv$ on $S$ and $s \in S$ we denote with $[s]_{\equiv}$ the equivalence class of $\equiv$ containing $s$. For a subset $T \subseteq S$ let $[T]_{\equiv}=\bigcup_{s \in T}[s]_{\equiv}$. The boolean closure of a set $F \subseteq \mathcal{P}(S)$ is denoted by $\operatorname{Bool}(F)$. It contains all subsets of $S$, which can be obtained by finitely many applications of complement and binary union, starting with subsets from $F$.

A semi-Thue system ST over the (not necessarily finite) alphabet $X$ is just a (possibly infinite) subset of $X^{*} \times X^{*}$. We associate with ST the one-step rewrite relation $\rightarrow \mathrm{sT}$, where $x \rightarrow \mathrm{sT} y$ for $x, y \in X^{*}$ if and only if there are $u, v \in X^{*}$ and $(\ell, r) \in \mathrm{ST}$ such that $x=u \ell v$ and $y=u r v$. The set of irreducible words with respect to $\mathrm{ST}$ is

$$
\operatorname{Irr}(\mathrm{ST})=\left\{x \in X^{*} \mid \neg \exists y \in X^{*}: x \rightarrow \mathrm{ST} y\right\} .
$$

A binary relation $\rightarrow$ over a set $E$ is confluent if for all $e, f, f^{\prime} \in E$ :

$$
\left(e \rightarrow^{*} f \wedge e \rightarrow^{*} f^{\prime}\right) \Rightarrow\left(\exists g \in E: f \rightarrow^{*} g \wedge f^{\prime} \rightarrow^{*} g\right)
$$

Suppose that a semi-Thue system ST has no overlapping left-hand sides, i.e., for all $(\ell, r),\left(\ell^{\prime}, r^{\prime}\right) \in \mathrm{ST}$ and $u, v \in X^{*}$ we have

$$
\left(u \ell=\ell^{\prime} v\right) \Rightarrow|u| \geq\left|\ell^{\prime}\right| \quad \text { and } \quad\left(u \ell v=\ell^{\prime}\right) \Rightarrow\left(u=v=\varepsilon \wedge r=r^{\prime}\right) .
$$


Then it can be easily shown that the relation $\rightarrow \mathrm{sT}$ is confluent. See [DJ91] for more information about confluence and other properties of rewriting systems.

\subsection{Finite automata over a free monoid}

Let $X$ be an alphabet (that we do not assume to be finite, in general). A finite automaton over the free monoid $X^{*}$ is a 5 -tuple

$$
\mathcal{A}=\langle\mathcal{X}, \mathrm{Q}, \delta, \mathrm{I}, \mathrm{T}\rangle,
$$

where $\mathcal{X}$ is a finite subset of $X, \mathrm{Q}$ is the finite set of states, $\mathrm{I} \subseteq \mathrm{Q}$ is the set of initial states, $\mathrm{T} \subseteq \mathrm{Q}$ is the set of terminal states, and $\delta$, the set of transitions, is a subset of $\mathrm{Q} \times \mathcal{X} \times \mathrm{Q}$. Let

$$
\mathrm{BR}(\mathrm{Q})=\mathcal{P}(\mathrm{Q} \times \mathrm{Q})
$$

be the monoid of binary relations over the set $\mathrm{Q}$. We recall that, given two binary relations $m, m^{\prime} \in \mathrm{BR}(\mathrm{Q})$, their composition is defined by

$$
m \circ m^{\prime}=\left\{\left(q, q^{\prime \prime}\right) \in \mathbf{Q} \times \mathbf{Q} \mid \exists q^{\prime} \in \mathbf{Q}:\left(q, q^{\prime}\right) \in m \wedge\left(q^{\prime}, q^{\prime \prime}\right) \in m^{\prime}\right\} .
$$

The automaton (3) induces a representation map

$$
\mu_{\mathcal{A}}:\left(X^{*}, \cdot, \varepsilon\right) \rightarrow\left(\operatorname{BR}(\mathrm{Q}), \circ, \operatorname{Id}_{\mathbf{Q}}\right)
$$

defined as the unique monoid homomorphism such that for every $x \in X$ :

$$
\mu_{\mathcal{A}}(x)=\{(q, r) \in \mathrm{Q} \times \mathrm{Q} \mid(q, x, r) \in \delta\} .
$$

We denote by $\odot$ the natural right-action of the monoid $B R(Q)$ over $\mathcal{P}(Q)$, i.e., for every $P \subseteq \mathrm{Q}$ and $m \in \mathrm{BR}(\mathrm{Q})$ let

$$
P \odot m=\{q \in \mathrm{Q} \mid \exists p \in P:(p, q) \in m\} .
$$

The language recognized by $\mathcal{A}$ is

$$
\mathrm{L}(\mathcal{A})=\left\{u \in X^{*} \mid \mu_{\mathcal{A}}(u) \cap(\mathbf{I} \times \mathbf{T}) \neq \emptyset\right\} .
$$

A subset $L \subseteq X^{*}$ is called regular if and only if there exists some finite automaton $\mathcal{A}$ such that $L=\mathrm{L}(\mathcal{A})$. Given a subset $Y \subseteq X$ and two states $p, q \in \mathrm{Q}$, the automaton $\mathcal{A}[Y, p, q]$ over $Y^{*}$ is defined by

$$
\mathcal{A}[Y, p, q]=\langle\mathcal{X} \cap Y, \mathrm{Q}, \delta \cap(\mathrm{Q} \times Y \times \mathrm{Q}),\{p\},\{q\}\rangle .
$$

\subsection{Rational subsets of a monoid}

Let $\mathbb{M}$ be a monoid together with a generating set $X$. Thus $\mathbb{M}=X^{*} / \equiv$, for some monoid congruence $\equiv$ over $X^{*}$. We denote by

$$
\pi: X^{*} \rightarrow X^{*} / \equiv
$$

the canonical homomorphism with $\pi(u)=[u]_{\equiv}$ for $u \in X^{*}$. 
The set

$$
\operatorname{Rat}(\mathbb{M}) \subseteq \mathcal{P}(M)
$$

is the smallest subset of $\mathcal{P}(M)$ which contains the finite subsets of $\mathbb{M}$ and which is closed under the operations $\cup$ (the union operation), · (the product operation) and * (the star operation, associating with a subset $P \subseteq \mathbb{M}$ the smallest submonoid of $\mathbb{M}$ containing $P$ ).

One can derive from Kleene's theorem about rational subsets of $X^{*}$ that the rational subsets of $\mathbb{M}$ are exactly the subsets of the form $\pi(\mathrm{L}(\mathcal{A}))$, where $\mathcal{A}$ is a finite automaton over the free monoid $X^{*}$. For such an automaton $\mathcal{A}=\langle\mathcal{X}, \mathrm{Q}, \delta, \mathrm{I}, \mathrm{T}\rangle$ let the representation map

$$
\mu_{\mathcal{A}, \mathbb{M}}: \mathbb{M} \rightarrow\left(\mathrm{BR}(\mathrm{Q}), \circ, \mathrm{Id}_{\mathrm{Q}}\right)
$$

by defined by

$$
\mu_{\mathcal{A}, \mathbb{M}}(m)=\bigcup\left\{\mu_{\mathcal{A}}(u) \mid u \in X^{*}, \pi(u)=m\right\}
$$

for $m \in \mathbb{M}$. Notice that

$$
\operatorname{Id}_{\mathbf{Q}} \subseteq \mu_{\mathcal{A}, \mathbb{M}}(1) \text { and } \mu_{\mathcal{A}, \mathbb{M}}(m) \circ \mu_{\mathcal{A}, \mathbb{M}}\left(m^{\prime}\right) \subseteq \mu_{\mathcal{A}, \mathbb{M}}\left(m \cdot m^{\prime}\right)
$$

but in general the map $\mu_{\mathcal{A}, \mathbb{M}}$ is not a monoid homomorphism. In Section 4.1, we introduce in the particular case where $\mathbb{M}$ is an HNN-extension a new kind of finite automaton, called finite t-automata, which do not even fulfil property (6). We will show that, nevertheless, we can always normalize finite $t$-automata in such a way that the map $\mu$ fulfils some nice algebraic property, see Definition 9 and Proposition 33 .

Anissimov and Seifert have shown:

Theorem 1 ([AS75]). Let $G$ be a group. The rational subgroups of $G$ are exactly the finitely generated subgroups of $G$.

We present a proof sketch for Theorem 1, because the underlying idea will be used later in a slightly modified form.

Proof sketch for Theorem 1. Let $G=X^{*} / \equiv$ be a group, where $X$ is some alphabet and $\equiv$ is a congruence over $X^{*}$. We suppose that $X$ is endowed with an involution $x \mapsto x^{-1}$ such that for every $x \in X, x x^{-1} \equiv x^{-1} x \equiv \varepsilon$. We extend this involution to $X^{*}$ by setting $\left(x_{1} \cdots x_{n}\right)^{-1}=x_{n}^{-1} \cdots x_{1}^{-1}$, where $x_{1}, \ldots, x_{n} \in X$. We denote by $\pi: X^{*} \rightarrow G$ the canonical projection, i.e., $\pi(u)=[u]_{\equiv}$. Let $\mathcal{A}=$ $\langle\mathcal{X}, \mathrm{Q}, \delta, \mathrm{I}, \mathrm{T}\rangle$ be some finite automaton over $X^{*}$ such that

$$
\pi(\mathrm{L}(\mathcal{A}))=K
$$

is a subgroup of $G$. Without loss of generality, we can suppose that $\mathcal{A}$ has a unique initial state $q_{0}$ and that $\mathcal{A}$ is trim, i.e., that every state is accessible from $\mathrm{I}=\left\{q_{0}\right\}$ 
and co-accessible from T. Let us define $\delta^{-1}=\left\{\left(r, x^{-1}, q\right) \mid(q, x, r) \in \delta\right\}$ and $\delta^{\prime}=\delta \cup \delta^{-1}$. Since $\pi(\mathrm{L}(\mathcal{A}))=K$ is a subgroup of $G$, it follows easily that

$$
\pi(\mathrm{L}(\mathcal{A}))=\pi\left(\mathrm{L}\left(\left\langle\mathcal{X}, \mathrm{Q}, \delta^{\prime}, \mathrm{I}, \mathrm{T}\right\rangle\right)\right) .
$$

Let us fix some subset $\lambda \subseteq \delta^{\prime}$ which is a covering tree in the sense that for every $q \in \mathrm{Q}$, there exists a unique path $\Lambda(q)$ from $q_{0}$ to $q$, using only edges in $\lambda$. We denote by $W(q) \in X^{*}$ the sequence of labels of this path. Note that $W\left(q_{0}\right)=\varepsilon$. Let us define

$$
\mathcal{G}=\left\{W(q) x W(r)^{-1} \mid(q, x, r) \in \delta\right\} \cup\{W(q) \mid q \in \mathrm{T}\} \subseteq X^{*} .
$$

One can check that $K=\pi\left(\left(\mathcal{G} \cup \mathcal{G}^{-1}\right)^{*}\right)$. Thus, $K$ is finitely generated.

The idea above is reminiscent of that of defining a set of generators for the fundamental group of a graph, see e.g. [Ser03, Section 5.1].

Definition 2 (Howson property). A group $G$ is said to have the Howson property if for every two finitely generated subgroups $K_{1}$ and $K_{2}$ of $G$, the subgroup $K_{1} \cap K_{2}$ is finitely generated, too.

Definition 3 (subgroup separation property, LERF). A group $G$ is said to have the subgroup separation property (also called local extended residual finiteness property - LERF for short) if for every finitely generated proper subgroup $K<G$ and every element $g \in G \backslash K$, there exists a subgroup $Z$ which has finite index in $G$ and such that $K \leq Z$ and $g \notin Z$.

The LERF property has been investigated in several works, see e.g. [AG73,Git97, Hal49,MR04,Sco78,Wis00].

Remark 4. The following two conditions are easily seen to be equivalent to the LERF property for $G$, see e.g. [MR04,Wis00]:

- For every finitely generated proper subgroup $K<G$ and every element $g \in G \backslash K$, there exists a normal subgroup $N$ which has finite index in $G$ and such that $g \notin N K$.

- For every finitely generated proper subgroup $K<G$ and every element $g \in G \backslash K$, there exists a homomorphism $\pi: G \rightarrow S$ with $S$ a finite group such that $\pi(g) \notin \pi(K)$.

\subsection{Algorithmic problems}

Let $\mathbb{M}=X^{*} / \equiv$ be a countably-generated monoid, where $X$ is a countable generating set. Let $\pi: X^{*} \rightarrow X^{*} / \equiv$ be the canonical homomorphism. We assume $\mathbb{M}$ to be countably generated in order to have a finite description of elements of $\mathbb{M}$ by words over $X$

The word problem for $\mathbb{M}$ is the following decision problem: 
instance: words $u, v \in X^{*}$

question: $\pi(u)=\pi(v)$ ?

Beside the word problem we shall investigate the following (more general) decision problems:

The membership problem for rational subsets of $\mathbb{M}$ is the following decision problem:

instance: a finite automaton $\mathcal{A}$ over $X^{*}$ and a word $u \in X^{*}$.

question: $\pi(u) \in \pi(\mathrm{L}(\mathcal{A}))$ ?

The emptiness problem for boolean combinations of rational subsets of $\mathbb{M}$ is the following decision problem:

instance: a set $B \in \operatorname{Bool}(\operatorname{Rat}(\mathbb{M})$ ) (represented by a boolean combination $B$ of finite automata over $\left.X^{*}\right)$

question: $B=\emptyset$ ?

Let us now assume that $\mathbb{M}=X^{*} / \equiv$ is a group. The membership problem for finitely generated subgroups of $\mathbb{M}$, also called the generalized word problem (GWP for short), is the following decision problem:

instance: words $u_{1}, \ldots, u_{n}, u \in X^{*}$.

question: $\pi(u) \in\left\{\pi\left(u_{1}\right), \pi\left(u_{1}\right)^{-1}, \ldots, \pi\left(u_{n}\right), \pi\left(u_{n}\right)^{-1}\right\}^{*}$, i.e., does $\pi(u)$ belong to the subgroup of $\mathbb{M}$ generated by $\pi\left(u_{1}\right), \ldots, \pi\left(u_{n}\right)$ ?

It is easy to see that the decidability status of all these decision problems is independent of the chosen generating set $X$ for $\mathbb{M}$, i.e., they are indeed properties of $\mathbb{M}$.

\section{HNN-extensions}

Let us fix throughout this section a monoid $H$ (the base monoid). In this paper, a subgroup of $H$ is a submonoid of $H$, which forms a group. ${ }^{\text {a }}$ Equivalently, $A$ is a subgroup of $H$ if $A$ is a subgroup of the group of units of $H$. Let us also fix two finite, isomorphic subgroups $A \leq H, B \leq H$ and an isomorphism $\varphi: A \rightarrow B$. We also fix some presentation $\left\langle X ; \equiv_{H}\right\rangle$ of $H$, i.e., an alphabet $X$ and a congruence $\equiv_{H}$ over the free monoid $X^{*}$ such that $H=X^{*} / \equiv_{H}$. Note that $X$ is not supposed to be finite, in general. Without loss of generality we assume that every element $h \in A \cup B$ is of the form $[x]_{\equiv_{H}}$ for some letter $x \in X$. In particular, there is a letter 1 for the neutral element of $H$. Of course $1 \equiv_{H} \varepsilon$. We consider the HNN-extension

$$
G=\left\langle H, t ; t^{-1} a t=\varphi(a)(a \in A)\right\rangle .
$$

a Note that usually, a subgroup $A$ of $H$ is defined to be a subsemigroup of $H$, which forms a group. In particular, the identity element of $A$ may be an idempotent of $H$ different from the identity of $H$. Nevertheless, we prefer to use the term subgroup for our definition in order to avoid too many different notions. 
This means that $G=\left(X \cup\left\{t, t^{-1}\right\}\right)^{*} / \equiv$ where $\equiv$ is the smallest congruence over the free monoid $\left(X \cup\left\{t, t^{-1}\right\}\right)^{*}$ such that:

$$
\begin{aligned}
\equiv_{H} & \subseteq \equiv \\
t t^{-1} & \equiv t^{-1} t \equiv \varepsilon \\
t^{-1} a t & \equiv \varphi(a) \text { for all } a \in A
\end{aligned}
$$

Let

$$
\pi:\left(X \cup\left\{t, t^{-1}\right\}\right)^{*} \rightarrow G
$$

denote the canonical morphism defined by $\pi(w)=[w]_{\equiv}$ for $w \in\left(X \cup\left\{t, t^{-1}\right\}\right)^{*}$. This morphism naturally factorizes as

$$
\pi=\pi_{t} \circ \pi_{G}
$$

where

$$
\begin{aligned}
& \pi_{t}:\left(X \cup\left\{t, t^{-1}\right\}\right)^{*} \rightarrow H *\left\{t, t^{-1}\right\}^{*} \text { and } \\
& \pi_{G}: H *\left\{t, t^{-1}\right\}^{*} \rightarrow G .
\end{aligned}
$$

The kernel of $\pi_{G}$ coincides with the smallest congruence $\approx$ over $H *\left\{t, t^{-1}\right\}^{*}$ such that:

$$
\begin{aligned}
t t^{-1} & \approx t^{-1} t \approx 1 \\
a t & \approx t \varphi(a) \quad \text { for all } a \in A \\
b t^{-1} & \approx t^{-1} \varphi^{-1}(b) \text { for all } b \in B
\end{aligned}
$$

Note that (12) and (13) together imply (14) but below we will need (13) and (14) without assuming (12). Clearly, $G=H *\left\{t, t^{-1}\right\}^{*} / \approx$.

An element of $s \in H *\left\{t, t^{-1}\right\}^{*}$ can be viewed as a word over the alphabet $H \cup\left\{t, t^{-1}\right\}$ which has the form

$$
s=h_{0} t^{\alpha_{1}} h_{1} \cdots t^{\alpha_{i}} h_{i} \cdots t^{\alpha_{n}} h_{n},
$$

where $n \geq 0, \alpha_{i} \in\{1,-1\}$, and $h_{i} \in H$. Such an element $s \in H *\left\{t, t^{-1}\right\}^{*}$ is also called a $t$-sequence. The $t$-sequence $s$ is said to be a reduced sequence if it neither contains a factor of the form $t^{-1} a t$ (with $a \in A$ ) nor $t b t^{-1}$ (with $b \in B$ ). Let

$$
\operatorname{Red}(H, t)=\left\{s \in H *\left\{t, t^{-1}\right\}^{*} \mid s \text { is reduced }\right\} .
$$

We define the binary relation $\stackrel{1}{\sim}$ over $H *\left\{t, t^{-1}\right\}^{*}$ as follows: For all $s, s^{\prime} \in H *$ $\left\{t, t^{-1}\right\}^{*}$ let

$$
\begin{aligned}
s \stackrel{1}{\sim} s^{\prime} \quad \Leftrightarrow \quad \exists s_{1}, s_{2} \in H *\left\{t, t^{-1}\right\}^{*}, a \in A, b \in B: \\
\left\{\begin{array}{l}
\left(s=s_{1} t s_{2} \wedge s^{\prime}=s_{1} a^{-1} t \varphi(a) s_{2}\right) \vee \\
\left(s=s_{1} t^{-1} s_{2} \wedge s^{\prime}=s_{1} b^{-1} t^{-1} \varphi^{-1}(b) s_{2}\right)
\end{array}\right\} .
\end{aligned}
$$


Let us denote by $\sim$ the congruence over $H *\left\{t, t^{-1}\right\}^{*}$ generated by all the rules of type (13) and (14) above. It coincides with the reflexive and transitive closure of $\stackrel{1}{\sim}$. Equivalently, if

$$
\begin{aligned}
s & =h_{0} t^{\alpha_{1}} h_{1} \cdots t^{\alpha_{n}} h_{n} \text { and } \\
s^{\prime} & =h_{0}^{\prime} t^{\alpha_{1}^{\prime}} h_{1}^{\prime} \cdots t^{\alpha_{m}^{\prime}} h_{m}^{\prime}
\end{aligned}
$$

(with $n, m \geq 0, \alpha_{i}, \alpha_{j}^{\prime} \in\{1,-1\}$ and $h_{i}, h_{j}^{\prime} \in H$ ), then $s \sim s^{\prime}$ if and only if $n=m$, $\alpha_{i}=\alpha_{i}^{\prime}$ for $1 \leq i \leq n$, and there exist $c_{1}, \ldots, c_{2 n} \in A \cup B$ such that:

- if $\alpha_{i}=1$ then $c_{2 i-1} \in A$ and $c_{2 i}=\varphi\left(c_{2 i-1}\right) \in B(1 \leq i \leq n)$

- if $\alpha_{i}=-1$ then $c_{2 i} \in A$ and $c_{2 i-1}=\varphi\left(c_{2 i}\right) \in B(1 \leq i \leq n)$

- $h_{i} c_{2 i+1}=c_{2 i} h_{i}^{\prime}$ in $H$ for $0 \leq i \leq n$ (here we set $c_{0}=c_{2 n+1}=1$ )

This situation can be visualized by a diagram of the following form (also called a Van Kampen diagram, see [LS77] for more details), where $n=m=4$. Lightshaded (resp. dark-shaded) areas represent relations in $H$ (resp. relations of the form $a t=t \varphi(a)(a \in A)$ or $\left.b t^{-1}=t^{-1} \varphi^{-1}(b)(b \in B)\right)$.

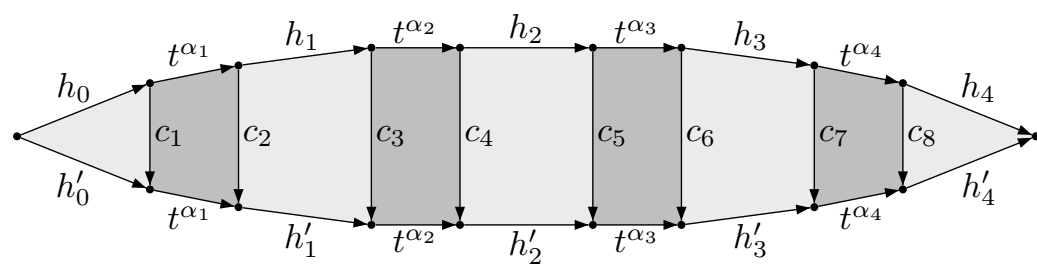

The elements $c_{1}, \ldots, c_{2 n}$ in such a diagram are also called connecting elements.

The set $\operatorname{Red}(H, t)$ is saturated by the congruence $\sim$, i.e., $s \sim s^{\prime}$ implies $s \in$ $\operatorname{Red}(H, t) \Leftrightarrow s^{\prime} \in \operatorname{Red}(H, t)$ : just notice that, since $A$ and $B$ are groups, $a h a^{\prime} \in$ $A \Leftrightarrow h \in A$ for all $h \in H, a, a^{\prime} \in A$ and $b h b^{\prime} \in B \Leftrightarrow h \in B$ for all $h \in H, b, b^{\prime} \in B$. This property would fail if $A$ and $B$ were assumed to be merely submonoids of $H$.

One has $\sim \subseteq \approx$. Moreover, for reduced sequences the following fundamental lemma holds.

Lemma 5. Let $s, s^{\prime} \in \operatorname{Red}(H, t)$. Then $s \approx s^{\prime}$ if and only if $s \sim s^{\prime}$.

In the case where $H$ is a group, Lemma 5 is an immediate consequence of [LS77, Theorem 2.1, p.182]. The extension to monoids can be proved in the same way as for groups using an idea of Artin and Van der Waerden (see Appendix A for a detailed verification). Lemma 5 implies that $H$ is a submonoid of $G$. Another consequence is:

Corollary 6. If $H$ has a decidable word problem, then also has $G$ a decidable word problem.

Proof. Let $w, w^{\prime} \in\left(X \cup\left\{t, t^{-1}\right\}\right)^{*}$ (recall that $X$ is a monoid generating set for $H)$. We describe a procedure for checking whether $w \equiv w^{\prime}$ or not. The words $w$ and 
$w^{\prime}$ have unique decompositions

$$
w=u_{0} t^{\alpha_{1}} u_{1} \cdots t^{\alpha_{i}} u_{i} \cdots t^{\alpha_{n}} u_{n} \text { and } w^{\prime}=u_{0}^{\prime} t^{\alpha_{1}^{\prime}} u_{1}^{\prime} \cdots t^{\alpha_{j}^{\prime}} u_{j}^{\prime} \cdots t^{\alpha_{m}^{\prime}} u_{m}^{\prime},
$$

where $n, m \geq 0, u_{i}, u_{j}^{\prime} \in X^{*}$, and $\alpha_{i}, \alpha_{j}^{\prime} \in\{1,-1\}$. Let the semi-Thue system ST over the alphabet $X \cup\left\{t, t^{-1}\right\}$ consist of the following rules:

$$
\begin{aligned}
& t^{-1} u t \rightarrow \varphi\left([u]_{\equiv_{H}}\right) \quad \text { for }[u]_{\equiv_{H}} \in A \\
& t u t^{-1} \rightarrow \varphi^{-1}\left([u]_{\equiv_{H}}\right) \text { for }[u]_{\equiv_{H}} \in B
\end{aligned}
$$

Note that $\operatorname{Red}(H, t)=\pi_{t}(\operatorname{Irr}(\mathrm{ST}))$. Since the word problem for $H$ is decidable and $A$ and $B$ are finite, we can effectively compute from $w$ and $w^{\prime}$ words $z$ and $z^{\prime}$ such that $w \rightarrow{ }_{\mathrm{ST}}^{*} z \in \operatorname{Irr}(\mathrm{ST})$ and $w \rightarrow \mathrm{ST}_{\mathrm{ST}}^{*} z^{\prime} \in \operatorname{Irr}(\mathrm{ST})$. Assume that

$$
z=v_{0} t^{\beta_{1}} v_{1} \cdots t^{\beta_{i}} v_{i} \cdots t^{\beta_{p}} v_{p} \text { and } z^{\prime}=v_{0}^{\prime} t^{\beta_{1}^{\prime}} v_{1}^{\prime} \cdots t^{\beta_{i}^{\prime}} v_{i}^{\prime} \cdots t^{\beta_{q}^{\prime}} v_{q}^{\prime} \text {, }
$$

where $p, q \geq 0, v_{i}, v_{j}^{\prime} \in X^{*}$, and $\beta_{i}, \beta_{j}^{\prime} \in\{1,-1\}$. Note that $\rightarrow \mathrm{sT} \subseteq \equiv$. Hence,

$$
\begin{aligned}
w \equiv w^{\prime} & \Leftrightarrow z \equiv z^{\prime} \\
& \Leftrightarrow \pi_{G}\left(\pi_{t}(z)\right)=\pi_{G}\left(\pi_{t}\left(z^{\prime}\right)\right) \\
& \Leftrightarrow \pi_{t}(z) \approx \pi_{t}\left(z^{\prime}\right) \\
& \Leftrightarrow \pi_{t}(z) \sim \pi_{t}\left(z^{\prime}\right),
\end{aligned}
$$

where the last equivalence holds due to Lemma 5 and the fact that $\pi_{t}(z), \pi_{t}\left(z^{\prime}\right) \in$ $\pi_{t}(\operatorname{Irr}(\mathrm{ST}))=\operatorname{Red}(H, t)$. If $p \neq q$ or $\left(p=q\right.$ and $\beta_{i} \neq \beta_{i}^{\prime}$ for some $\left.1 \leq i \leq p\right)$ in (18), then $\pi_{t}(z) \nsim \pi_{t}\left(z^{\prime}\right)$ and thus $w \neq \equiv w^{\prime}$. Hence, assume that

$$
z=v_{0} t^{\beta_{1}} v_{1} \cdots t^{\beta_{i}} v_{i} \cdots t^{\beta_{p}} v_{p} \text { and } z^{\prime}=v_{0}^{\prime} t^{\beta_{1}} v_{1}^{\prime} \cdots t^{\beta_{i}} v_{i}^{\prime} \cdots t^{\beta_{p}} v_{p}^{\prime} .
$$

We now guess the connecting elements between $\pi_{t}(z)$ and $\pi_{t}\left(z^{\prime}\right)$ in a diagram of the form ( $\dagger$ ). More precisely, we nondeterministically guess elements $c_{1}, \ldots, c_{2 n} \in A \cup B$ such that:

- if $\beta_{i}=1$ then $c_{2 i-1} \in A$ and $c_{2 i}=\varphi\left(c_{2 i-1}\right) \in B(1 \leq i \leq p)$ and

- if $\beta_{i}=-1$ then $c_{2 i} \in A$ and $c_{2 i-1}=\varphi\left(c_{2 i}\right) \in B(1 \leq i \leq p)$.

Since $A$ and $B$ are finite, this guessing can be done by a nondeterministic Turing machine. Finally, using the decidability of the word problem for $H$, we check whether $v_{i} c_{2 i+1} \equiv_{H} c_{2 i} v_{i}^{\prime}$ for $0 \leq i \leq p$ (here we set $c_{0}=c_{2 p+1}=1$ ). If this is true for some choice of $c_{1}, \ldots, c_{2 n} \in A \cup B$, then we know that indeed $w \equiv w^{\prime}$.

\subsection{HNN-extensions and morphisms}

Suppose we are given a monoid homomorphism: $\pi^{\prime}: H \rightarrow H^{\prime}$ fulfilling

$$
\operatorname{Ker}\left(\pi^{\prime}\right) \cap A \times A=\operatorname{Id}_{A} \text { and } \operatorname{Ker}\left(\pi^{\prime}\right) \cap B \times B=\operatorname{Id}_{B} .
$$

Thus, $A$ and $B$ are subgroups of $H^{\prime}$. Hence, we can define the HNN-extension

$$
G^{\prime}=\left\langle H^{\prime}, t ; t^{-1} a t=\varphi(a)(a \in A)\right\rangle .
$$




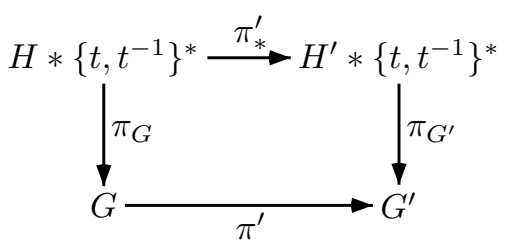

Fig. 1. Maps induced by $\pi^{\prime}$

In the presentation (20), every relation $t^{-1} a t=\varphi(a)$ should be written as $t^{-1} \pi^{\prime}(a) t=\pi^{\prime}(\varphi(a))$, but (19) allows us to identify every $a \in A$ with its image $\pi^{\prime}(a) \in \pi^{\prime}(A)$. The map $\pi^{\prime}$ induces a map $\pi_{*}^{\prime}: H *\left\{t, t^{-1}\right\}^{*} \rightarrow H^{\prime} *\left\{t, t^{-1}\right\}^{*}$, which is the unique monoid homomorphism such that

$$
\forall h \in H: \pi_{*}^{\prime}(h)=\pi^{\prime}(h), \quad \pi_{*}^{\prime}(t)=t, \quad \pi_{*}^{\prime}\left(t^{-1}\right)=t^{-1} .
$$

The composite morphism $\pi_{*}^{\prime} \circ \pi_{G^{\prime}}: H *\left\{t, t^{-1}\right\}^{*} \rightarrow G^{\prime}$ has a kernel which contains the congruence $\approx$. Hence, it induces a map $\widetilde{\pi}^{\prime}: G \rightarrow G^{\prime}$ such that

$$
\pi_{*}^{\prime} \circ \pi_{G^{\prime}}=\pi_{G} \circ \widetilde{\pi}^{\prime} .
$$

One can check that

$$
\forall h \in H: \widetilde{\pi}^{\prime}(h)=\pi^{\prime}(h), \quad \tilde{\pi}^{\prime}(t)=t, \quad \tilde{\pi}^{\prime}\left(t^{-1}\right)=t^{-1} .
$$

Hence $\widetilde{\pi}^{\prime}$ is an extension of $\pi^{\prime}$, therefore we shall (abusively) also use the symbol $\pi^{\prime}$ to denote $\widetilde{\pi}^{\prime}$. We represent the situation by the commutative diagram in Figure 1.

\section{Finite automata for $\mathrm{HNN}$-extensions}

Let us fix throughout this section an HNN-extension $G$ of a base monoid $H$ as described by (7) with $A$ and $B=\varphi(A)$ finite. We define below a special kind of finite automata, which will be well-suited for our aim of showing that some decidability and structural properties of rational subsets of $H$ are transfered to $G$.

\subsection{Finite t-automata}

We define here a kind of finite automata that recognize subsets of $H *\left\{t, t^{-1}\right\}^{*}$ via transitions that either read one of the letters $t, t^{-1}$ or read an element of $H$, provided it belongs to some definite set $F \in \mathcal{F}$. The typical class $\mathcal{F}$ that we have in mind is the set $\operatorname{Rat}(H)$ of all rational subsets of $H$, or the set $\mathcal{S}$ of all right-cosets of finitely generated subgroups of $H$ (in case $H$ is a group), or some other families of subsets of $H$ derived in a natural way from the two above families.

Let $\mathcal{F} \subseteq \mathcal{P}(H)$ be a set of subsets of $H$ such that

$$
\forall c \in A \cup B:\{c\} \in \mathcal{F} .
$$

A finite t-automaton, briefly fta, over $H *\left\{t, t^{-1}\right\}^{*}$ with labelling set $\mathcal{F}$ is a 5 -tuple

$$
\mathcal{A}=\langle\mathcal{L}, \mathrm{Q}, \delta, \mathrm{I}, \mathrm{T}\rangle
$$


where

$$
\mathcal{L} \subseteq \mathcal{F} \cup\left\{\{t\},\left\{t^{-1}\right\}\right\}
$$

is finite, $\mathrm{Q}$ is a finite set of states, $\mathrm{I} \subseteq \mathrm{Q}$ is the set of initial states, $\mathrm{T} \subseteq \mathrm{Q}$ is the set of terminal states, and $\delta$ (the set of transitions) is a subset of $\mathrm{Q} \times \mathcal{L} \times \mathrm{Q}$ such that

$$
\forall q \in \mathrm{Q} \exists L \subseteq \mathcal{L}: 1 \in L \wedge(q, L, q) \in \delta .
$$

We define

$$
\widehat{\delta}=\left\{(p, h, q) \in \mathrm{Q} \times\left(H \cup\left\{t, t^{-1}\right\}\right) \times \mathrm{Q} \mid \exists L \subseteq \mathcal{L}: h \in L \wedge(p, L, q) \in \delta\right\} .
$$

The automaton $\mathcal{A}$ induces a representation map

$$
\mu_{\mathcal{A}}: H *\left\{t, t^{-1}\right\}^{*} \rightarrow \mathrm{BR}(\mathrm{Q})
$$

defined as follows: First, define $\mu_{\mathcal{A}, 0}: H \cup\left\{t, t^{-1}\right\} \rightarrow \mathrm{BR}(\mathrm{Q})$ as follows, where $h \in H \cup\left\{t, t^{-1}\right\}:$

$$
\mu_{\mathcal{A}, 0}(h)=\{(q, r) \in Q \times Q \mid(q, h, r) \in \widehat{\delta}\}
$$

(note that $\operatorname{Id}_{\mathrm{Q}} \subseteq \mu_{\mathcal{A}, 0}(1)$ due to $(23)$ ). For $h \in H \cup\left\{t, t^{-1}\right\}$ and $q, r \in \mathrm{Q}$ we will also write $q \stackrel{h}{\rightarrow} \mathcal{A} r$ instead of $(q, r) \in \mu_{\mathcal{A}, 0}(h)$.

Now, let $s \in H *\left\{t, t^{-1}\right\}^{*}$ be a sequence of the form (15). Then

$$
\begin{aligned}
\mu_{\mathcal{A}}(s)=\mu_{\mathcal{A}, 0}\left(h_{0}\right) \circ \mu_{\mathcal{A}, 0}\left(t^{\alpha_{1}}\right) \circ \mu_{\mathcal{A}, 0}\left(h_{1}\right) & \cdots \\
& \mu_{\mathcal{A}, 0}\left(t^{\alpha_{i}}\right) \circ \mu_{\mathcal{A}, 0}\left(h_{i}\right) \cdots \mu_{\mathcal{A}, 0}\left(t^{\alpha_{n}}\right) \circ \mu_{\mathcal{A}, 0}\left(h_{n}\right) .
\end{aligned}
$$

Notice that for the definition of the map $\mu_{\mathcal{A}}$ we only consider paths in $\mathcal{A}$, which alternate strictly between $H$-edges and $t^{ \pm 1}$-edges. As a consequence of this definition, the second property stated in (6) need not be fulfilled by this kind of automata in case $m, m^{\prime} \in H$. Instead of $(q, r) \in \mu_{\mathcal{A}}(s)$ we also write $q \stackrel{s}{\rightarrow} \mathcal{A} r\left(\right.$ since $\mu_{\mathcal{A}, 0}(h)=\mu_{\mathcal{A}}(h)$ for $h \in H \cup\left\{t, t^{-1}\right\}$, this notation extends our notation introduced earlier).

The subset of $H *\left\{t, t^{-1}\right\}^{*}$ recognized by $\mathcal{A}$ is

$$
\mathrm{L}(\mathcal{A})=\left\{s \in H *\left\{t, t^{-1}\right\}^{*} \mid \mu_{\mathcal{A}}(s) \cap(\mathrm{I} \times \mathrm{T}) \neq \emptyset\right\} .
$$

Let us introduce a finite set $\mathcal{T}_{6}$ of 6 types, which will induce a partition of the states of an fta:

$$
\mathcal{T}_{6}=\{(A, T),(B, T),(1, H),(1,1),(A, H),(B, H)\}
$$

The first component of the types will serve below for defining a normal form for automata; the second component suggests the possible labels going out of a state of that type. We define a directed, edge-labeled graph $\mathcal{G}_{6}=\left(\mathcal{T}_{6}, \mathcal{E}_{6}\right)$ by

$$
\begin{aligned}
\mathcal{E}_{6}= & \left\{((C, D), C,(C, D)) \mid(C, D) \in \mathcal{T}_{6}\right\} \\
& \cup\left\{((A, T), t,(B, H)),\left((B, T), t^{-1},(A, H)\right)\right\} \\
& \cup\{((C, H), H,(C, T)),((1, H), H,(C, T)) \mid C \in\{A, B\}\} \\
& \cup\{((C, H), H,(1,1)) \mid C \in\{1, A, B\}\} \\
& \cup\{((B, H), H,(A, T)),((A, H), H,(B, T))\} .
\end{aligned}
$$




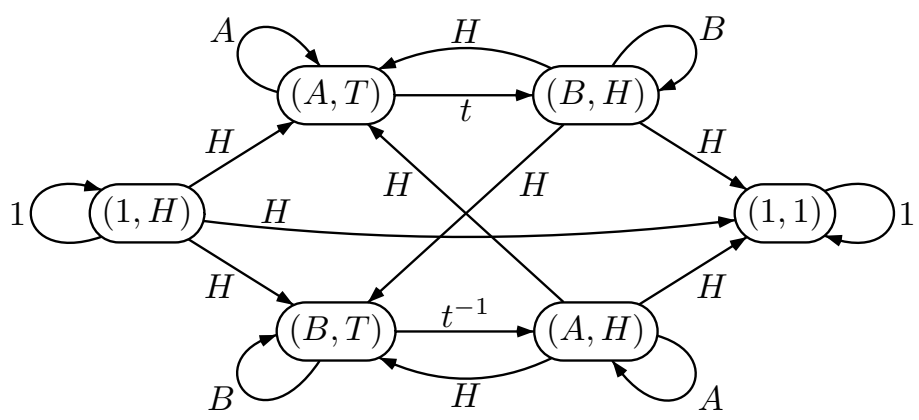

Fig. 2. The graph $\mathcal{G}_{6}=\left(\mathcal{I}_{6}, \mathcal{E}_{6}\right)$

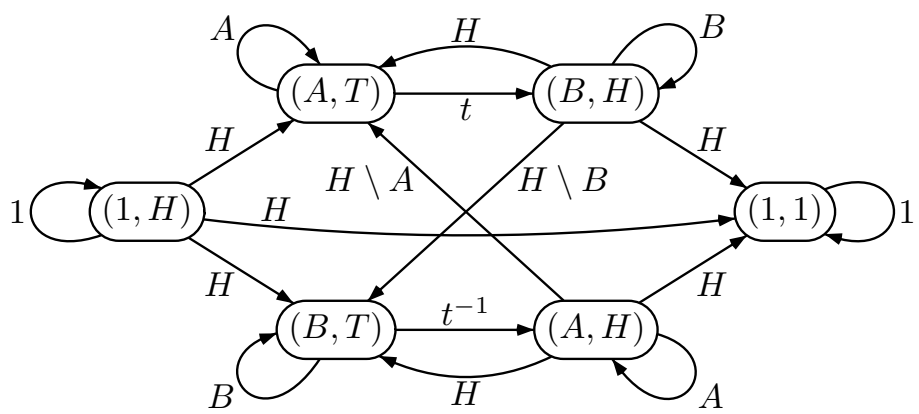

Fig. 3. The graph $\mathcal{R}_{6}=\left(\mathcal{T}_{6}, \mathcal{E}_{6}^{\prime}\right)$

The graph $\mathcal{G}_{6}$ is represented in Figure 2. We sometimes use also the graph $\mathcal{R}_{6}=$ $\left(\mathcal{T}_{6}, \mathcal{E}_{6}^{\prime}\right)$ where

$$
\begin{aligned}
\mathcal{E}_{6}^{\prime}= & \mathcal{E}_{6} \backslash\{((A, H), H,(A, T)),((B, H), H,(B, T))\} \cup \\
& \{((A, H), H \backslash A,(A, T)),((B, H), H \backslash B,(B, T))\} ;
\end{aligned}
$$

it is shown in Figure 3 . One can check that the graph $\mathcal{G}_{6}$ (resp. $\mathcal{R}_{6}$ ) endowed with the set of initial states $\mathbf{I}_{6}=\{(1, H)\}$ and the set of final states $\mathbf{T}_{6}=\{(1,1)\}$ is an fta recognizing $H *\left\{t, t^{-1}\right\}^{*}(\operatorname{resp}$. $\operatorname{Red}(H, t)) .{ }^{\mathrm{b}}$ For this, the $A$-loops and $B$-loops are not really relevant, but these loops will be useful later, when we introduce the property of $\sim$-saturation, see Definition 9.

Since $\mathcal{G}_{6}$ and $\mathcal{R}_{6}$ contain only a single $t$-transition (resp. $t^{-1}$-transition), one can easily check the following (recall the definition of the ${ }^{\wedge}$-operator from (24):

Lemma 7. For every sequence $s \in H *\left\{t, t^{-1}\right\}^{*}$ of the form (15) there is exactly one sequence $\Theta_{0}, \Theta_{1}, \ldots, \Theta_{2 n+1} \in \mathcal{T}_{6}$ of types such that

${ }^{\mathrm{b}}$ Formally, the label $t^{\alpha}(\alpha \in\{1,-1\})$ of $\mathcal{G}_{6}\left(\right.$ resp. $\left.\mathcal{R}_{6}\right)$ has to be replaced by a transition labelled with the singleton set $\left\{t^{\alpha}\right\}$ in order to make $\mathcal{G}_{6}\left(\right.$ resp. $\left.\mathcal{R}_{6}\right)$ an $\mathrm{fta}$. In the rest of this paper, we will repeatedly write $t^{\alpha}$, when we formally have to write $\left\{t^{\alpha}\right\}$. 
(1) $\Theta_{0}=(1, H), \Theta_{2 n+1}=(1,1)$,

(2) $\left(\Theta_{2 i}, h_{i}, \Theta_{2 i+1}\right) \in \widehat{\mathcal{E}}_{6}$ for all $0 \leq i \leq n$, and

(3) $\left(\Theta_{2 i-1}, t_{i}^{\alpha_{i}}, \Theta_{2 i}\right) \in \widehat{\mathcal{E}}_{6}$ for all $1 \leq i \leq n$.

Moreover, we must have $\Theta_{i} \neq \Theta_{i+1}$ for all $0 \leq i \leq 2 n$.

Lemma 7 implies that that every sequence $s$ can be accepted in a unique way by the fta $\mathcal{G}_{6}$. The same statement also holds for $\mathcal{R}_{6}$ if we restrict to reduced sequences.

Definition 8 (partitionned fta). A partitioned fta with labelling set $\mathcal{F}$ is a 6 tuple

$$
\mathcal{A}=\langle\mathcal{L}, \mathrm{Q}, \tau, \delta, \mathrm{I}, \mathrm{T}\rangle,
$$

where $\mathcal{L}, \mathrm{Q}, \delta, \mathrm{I}$, and $\mathrm{T}$ are as in (21), and $\tau: Q \rightarrow \mathcal{T}_{6}$ assigns a type to every state, and the transitions of $\delta$ and the sets $I$ and $\mathrm{T}$ respect the types in the following sense:

$$
\begin{aligned}
& \forall(q, h, r) \in \widehat{\delta}:(\tau(q), h, \tau(r)) \in \widehat{\mathcal{E}}_{6} \\
& \tau(\mathrm{I})=\{(1, H)\} \wedge \tau(\mathrm{T})=\{(1,1)\} .
\end{aligned}
$$

Recall the equivalences $\sim$ and $\approx$ over $H *\left\{t, t^{-1}\right\}^{*}$ defined in Section 3.

Definition 9 ( $\approx$-compatible, $\sim$-saturated). An fta $\mathcal{A}$ is said to be $\approx$-compatible if and only if

$$
[\mathrm{L}(\mathcal{A})]_{\approx}=[\mathrm{L}(\mathcal{A}) \cap \operatorname{Red}(H, t)]_{\approx} .
$$

It is said to be $\sim$-saturated if and only if

$$
\forall s, s^{\prime} \in H *\left\{t, t^{-1}\right\}^{*}: s \sim s^{\prime} \Rightarrow \mu_{\mathcal{A}}(s)=\mu_{\mathcal{A}}\left(s^{\prime}\right) .
$$

Remark 10. The following observations are easy to verify:

- Property (31) is equivalent to $\mathrm{L}(\mathcal{A}) \subseteq[\mathrm{L}(\mathcal{A}) \cap \operatorname{Red}(H, t)] \approx$, i.e., for every sequence $s \in \mathrm{L}(\mathcal{A})$ there must exist a reduced sequence $s^{\prime} \in \mathrm{L}(\mathcal{A})$ such that $s \approx s^{\prime}$.

- If $\mathrm{L}(\mathcal{A}) \subseteq \operatorname{Red}(H, t)$, then $\mathcal{A}$ is $\approx$-compatible.

- $\mathcal{A}$ is $\sim$-saturated if and only if for all states $q_{1}, q_{2}, q_{3}, q_{4} \in \mathrm{Q}$, all $h_{1}, h_{2} \in H$, and all $a \in A, b \in B$ such that

$$
\begin{gathered}
q_{1} \stackrel{h_{1}}{\longrightarrow} \mathcal{A} q_{2} \stackrel{t}{\rightarrow} \mathcal{A} q_{3} \stackrel{h_{2}}{\longrightarrow} \mathcal{A} q_{4} \\
\text { (resp. } q_{1} \stackrel{h_{1}}{\longrightarrow} \mathcal{A} q_{2} \stackrel{t^{-1}}{\longrightarrow} \mathcal{A} q_{3} \stackrel{h_{2}}{\longrightarrow} \mathcal{A} q_{4} \text { ), }
\end{gathered}
$$

there exist $q_{2}^{\prime}, q_{3}^{\prime} \in \mathrm{Q}$ with

$$
\begin{gathered}
q_{1} \stackrel{h_{1} a^{-1}}{\longrightarrow} \mathcal{A} q_{2}^{\prime} \stackrel{t}{\longrightarrow}_{\mathcal{A}} q_{3}^{\prime} \stackrel{\varphi(a) h_{2}}{\longrightarrow} \mathcal{A} q_{4} \\
\left(\text { resp. } q_{1} \stackrel{h_{1} b^{-1}}{\longrightarrow} \mathcal{A} q_{2}^{\prime} \stackrel{t^{-1}}{\longrightarrow} \mathcal{A} q_{3}^{\prime} \stackrel{\varphi^{-1}(b) h_{2}}{\longrightarrow} \mathcal{A} q_{4}\right) .
\end{gathered}
$$


- Whereas $\approx$-compatibility depends on $\mathrm{L}(\mathcal{A})$ only, $\sim$-saturation does really depend on the automaton $\mathcal{A}$ itself.

- If $\mathcal{A}$ is $\sim$-saturated, then the set $\mathrm{L}(\mathcal{A})$ is $\sim$-saturated, i.e., if $s \sim s^{\prime}$, then $s \in \mathrm{L}(\mathcal{A})$ if and only if $s^{\prime} \in \mathrm{L}(\mathcal{A})$.

- The partitioned fta $\mathcal{G}_{6}$ and $\mathcal{R}_{6}$ are both $\approx$-compatible and $\sim$-saturated. Since we require (23), the $A$-labeled (resp. $B$-labeled) loops at state $(A, T)$ and $(A, H)$ (resp. $(B, T)$ and $(B, H))$ in Figure 2 and 3 are crucial for $\sim$-saturations of $\mathcal{G}_{6}$ and $\mathcal{R}_{6}$.

Definition 11 (unitary fta). The partitioned fta $\mathcal{A}=\langle\mathcal{L}, \mathrm{Q}, \tau, \delta, \mathrm{I}, \mathrm{T}\rangle$ is said to be unitary if and only if for every type $\Theta \in \mathcal{T}_{6}$ we have

$$
\mu_{\mathcal{A}, 0}(1) \cap\left(\tau^{-1}(\Theta) \times \tau^{-1}(\Theta)\right)=\operatorname{Id}_{Q} \cap\left(\tau^{-1}(\Theta) \times \tau^{-1}(\Theta)\right) .
$$

This last technical condition turns out to be useful in [LS06a]. It means that no edge between two different states of the same type is labelled with a set containing 1.

Definition 12 (deterministic fta). The partitioned fta $\mathcal{A}=\langle\mathcal{L}, \mathrm{Q}, \tau, \delta, \mathrm{I}, \mathrm{T}\rangle$ is said to be deterministic if and only if

$$
\operatorname{Card}(\mathrm{I})=1
$$

(i.e. it has exactly one initial state) and for all $q, r, r^{\prime} \in \mathrm{Q}$ and all $L, L^{\prime} \in \mathcal{L}$,

$$
\left((q, L, r),\left(q, L^{\prime}, r^{\prime}\right) \in \delta \wedge L \cap L^{\prime} \neq \emptyset \wedge \tau(r)=\tau\left(r^{\prime}\right)\right) \Rightarrow\left(L=L^{\prime} \wedge r=r^{\prime}\right) .
$$

Clearly, $\mathcal{G}_{6}$ and $\mathcal{R}_{6}$ are both deterministic, since these fta both have only one initial state and for every type there exists only one state of that type.

Finally, we define the notion of a complete partitioned fta. The intuitive idea is that of a local condition over the labels of transitions, which ensures that for every reduced sequence $s \in \operatorname{Red}(H, t)$ and every state $q$ of type $(1, H)$, we have $q \stackrel{s}{\rightarrow} \mathcal{A} r$ for some state $r$ of type $(1,1)$. For every $q \in \mathrm{Q}$ and every $\Theta \in \mathcal{I}_{6}$ we thus introduce the set

$$
\mathrm{L}^{1}(q, \Theta)=\bigcup\left\{L \mid \exists r \in \tau^{-1}(\Theta):(q, L, r) \in \delta\right\} .
$$

Definition 13 (complete fta). The partitioned fta $\mathcal{A}=\langle\mathcal{L}, \mathrm{Q}, \tau, \delta, \mathrm{I}, \mathrm{T}\rangle$ is said to be complete if and only if

$$
\forall\left(\Theta, C, \Theta^{\prime}\right) \in \mathcal{E}_{6}^{\prime} \forall q \in \tau^{-1}(\Theta): C \subseteq \mathrm{L}^{1}\left(q, \Theta^{\prime}\right) .
$$

Note that $\mathcal{G}_{6}$ and $\mathcal{R}_{6}$ are both complete. 


\subsection{Operations on fta}

In this section we introduce operations on fta, which are analogues of the classical complement and product construction for finite automata.

Definition 14 (product automaton). Given two fta $\mathcal{A}_{i}=\left\langle\mathcal{L}_{i}, \mathrm{Q}_{i}, \delta_{i}, \mathrm{I}_{i}, \mathrm{~T}_{i}\right\rangle(i \in$ $\{1,2\})$, we define the product fta $\mathcal{A}_{1} \times \mathcal{A}_{2}=\langle\mathcal{L}, \mathrm{Q}, \delta, \mathrm{I}, \mathrm{T}\rangle$, where:

$$
\begin{aligned}
\mathcal{L} & =\left\{L_{1} \cap L_{2} \mid L_{1} \in \mathcal{L}_{1}, L_{2} \in \mathcal{L}_{2}\right\} \backslash\{\emptyset\} \\
\mathrm{Q} & =\mathrm{Q}_{1} \times \mathrm{Q}_{2}, \\
\mathrm{I} & =\mathrm{I}_{1} \times \mathrm{I}_{2} \\
\mathrm{~T} & =\mathrm{T}_{1} \times \mathrm{T}_{2} \\
\delta & =\left\{\left(\left(p_{1}, p_{2}\right), L_{1} \cap L_{2},\left(q_{1}, q_{2}\right)\right) \mid\left(p_{1}, L_{1}, q_{1}\right) \in \delta_{1},\left(p_{2}, L_{2}, q_{2}\right) \in \delta_{2}\right\}
\end{aligned}
$$

For two partitioned fta $\mathcal{A}_{i}=\left\langle\mathcal{L}_{i}, \mathrm{Q}_{i}, \tau_{i}, \delta_{i}, \mathrm{I}_{i}, \mathrm{~T}_{i}\right\rangle$ the product automaton is the partitioned fta $\mathcal{A}_{1} \times \mathcal{A}_{2}=\langle\mathcal{L}, \mathrm{Q}, \tau, \delta, \mathrm{I}, \mathrm{T}\rangle$, where $\mathcal{L}, \mathrm{I}$, and $\mathrm{T}$ are defined as above, but

$$
\begin{aligned}
& \mathbf{Q}=\bigcup_{\Theta \in \mathcal{T}_{6}} \tau_{1}^{-1}(\Theta) \times \tau_{2}^{-1}(\Theta), \\
& \delta=\left\{\left(\left(p_{1}, p_{2}\right), L_{1} \cap L_{2},\left(q_{1}, q_{2}\right)\right) \mid\left(p_{1}, L_{1}, q_{1}\right) \in \delta_{1},\left(p_{2}, L_{2}, q_{2}\right) \in \delta_{2}\right\} \cap \mathbf{Q} \times \mathcal{L} \times \mathbf{Q}, \\
& \text { and } \tau\left(p_{1}, p_{2}\right)=\tau_{1}\left(p_{1}\right)=\tau_{2}\left(p_{2}\right) \text { for }\left(p_{1}, p_{2}\right) \in \mathbf{Q} .
\end{aligned}
$$

Lemma 15. For two fta (resp. partitioned fta) $\mathcal{A}_{1}$ and $\mathcal{A}_{2}$ we have $\mathrm{L}\left(\mathcal{A}_{1} \times \mathcal{A}_{2}\right)=$ $\mathrm{L}\left(\mathcal{A}_{1}\right) \cap \mathrm{L}\left(\mathcal{A}_{2}\right)$.

Proof. For ordinary (non-partitioned) fta the statement of the lemma is obvious. For partitioned fta one has to use Lemma 7. It implies that if $s \in \mathrm{L}\left(\mathcal{A}_{1}\right) \cap \mathrm{L}\left(\mathcal{A}_{2}\right)$, then the state sequences in accepting runs of $\mathcal{A}_{1}$ and $\mathcal{A}_{2}$, respectively, on $s$ are mapped to the same sequence of types via the type functions of $\mathcal{A}_{1}$ and $\mathcal{A}_{2}$, respectively.

Lemma 15 implies:

Lemma 16. Let $\mathcal{A}$ be some fta with labelling set $\mathcal{F}$. The fta $\mathcal{A} \times \mathcal{G}_{6}$ endowed with the map $\tau:(p, \Theta) \mapsto \Theta$ is a partitioned fta with labelling set $\mathcal{F}$ and $\mathrm{L}\left(\mathcal{A} \times \mathcal{G}_{6}\right)=\mathrm{L}(\mathcal{A})$.

Lemma 17. Let $\mathcal{A}_{1}$ and $\mathcal{A}_{2}$ be fta (resp. partitioned fta). Then we have:

(a) If $\mathcal{A}_{1}$ and $\mathcal{A}_{2}$ are both $\sim$-saturated, then $\mathcal{A}_{1} \times \mathcal{A}_{2}$ is $\sim$-saturated, too.

(b) If $\mathcal{A}_{1}$ and $\mathcal{A}_{2}$ are both $\approx$-compatible and $\sim$-saturated, then $\mathcal{A}_{1} \times \mathcal{A}_{2}$ is $\approx$ compatible and $\sim$-saturated, too.

Proof. For statement (a) and non-partitioned fta assume that in the product automaton $\mathcal{A}=\mathcal{A}_{1} \times \mathcal{A}_{2}$ we have

$$
\left(q_{1}, p_{1}\right){\stackrel{h_{1}}{\longrightarrow}}_{\mathcal{A}}\left(q_{2}, p_{2}\right) \stackrel{t}{\rightarrow}_{\mathcal{A}}\left(q_{3}, p_{3}\right){\stackrel{h_{2}}{\longrightarrow}}_{\mathcal{A}}\left(q_{4}, p_{4}\right) .
$$


Hence,

$$
\begin{aligned}
& q_{1} \stackrel{h_{1}}{\longrightarrow} \mathcal{A}_{1} q_{2} \stackrel{t}{\rightarrow} \mathcal{A}_{1} q_{3} \stackrel{h_{2}}{\longrightarrow} \mathcal{A}_{1} q_{4} \text { and } \\
& p_{1} \stackrel{h_{1}}{\longrightarrow} \mathcal{A}_{2} p_{2} \stackrel{t}{\rightarrow} \mathcal{A}_{2} p_{3} \stackrel{h_{2}}{\longrightarrow} \mathcal{A}_{2} p_{4} .
\end{aligned}
$$

Since $\mathcal{A}_{1}$ and $\mathcal{A}_{2}$ are both $\sim$-saturated, we have, for every $a \in A$,

$$
\begin{aligned}
& q_{1} \stackrel{h_{1} a^{-1}}{\longrightarrow} \mathcal{A}_{1} q_{2}^{\prime} \stackrel{t}{\rightarrow} \mathcal{A}_{1} q_{3}^{\prime} \stackrel{\varphi(a) h_{2}}{\longrightarrow} \mathcal{A}_{1} q_{4} \text { and } \\
& p_{1} \stackrel{h_{1} a^{-1}}{\longrightarrow} \mathcal{A}_{2} p_{2}^{\prime} \stackrel{t}{\longrightarrow} \mathcal{A}_{2} p_{3}^{\prime} \stackrel{\varphi(a) h_{2}}{\longrightarrow} \mathcal{A}_{2} p_{4}
\end{aligned}
$$

for states $q_{2}^{\prime}$ and $q_{3}^{\prime}$ (resp. $p_{2}^{\prime}$ and $p_{3}^{\prime}$ ) of $\mathcal{A}_{1}$ (resp. $\mathcal{A}_{2}$ ). Hence,

$$
\left(q_{1}, p_{1}\right) \stackrel{h_{1} a^{-1}}{\longrightarrow} \mathcal{A}\left(q_{2}^{\prime}, p_{2}^{\prime}\right) \stackrel{t}{\rightarrow} \mathcal{A}\left(q_{3}^{\prime}, p_{3}^{\prime}\right) \stackrel{\varphi(a) h_{2}}{\longrightarrow} \mathcal{A}\left(q_{4}, p_{4}\right) .
$$

An analogous argument for $t^{-1}$ shows that $\mathcal{A}=\mathcal{A}_{1} \times \mathcal{A}_{2}$ is indeed $\sim$-saturated. For partitioned fta we can reason similarly, we just have to note that the states $q_{2}, q_{2}^{\prime}, p_{2}, p_{2}^{\prime}$ (resp. $\left.q_{3}, q_{3}^{\prime}, p_{3}, p_{3}^{\prime}\right)$ have to be of type $(A, T)$ (resp. $\left.(B, H)\right)$. This, ensures that $\left(q_{2}^{\prime}, p_{2}^{\prime}\right)$ and $\left(q_{3}^{\prime}, p_{3}^{\prime}\right)$ are states of the partitioned fta $\mathcal{A}$.

For (b) it suffices by Lemma 15 and statement (a) to prove that $\mathrm{L}\left(\mathcal{A}_{1}\right) \cap \mathrm{L}\left(\mathcal{A}_{2}\right) \subseteq$ $\left[\mathrm{L}\left(\mathcal{A}_{1}\right) \cap \mathrm{L}\left(\mathcal{A}_{2}\right) \cap \operatorname{Red}(H, t)\right]_{\approx}$. So, assume that

$$
s \in \mathrm{L}\left(\mathcal{A}_{1}\right) \cap \mathrm{L}\left(\mathcal{A}_{2}\right) .
$$

Since $\mathcal{A}_{1}$ and $\mathcal{A}_{2}$ are both $\approx$-compatible, we have

$$
s \in\left[\mathrm{L}\left(\mathcal{A}_{1}\right) \cap \operatorname{Red}(H, t)\right] \approx \cap\left[\mathrm{L}\left(\mathcal{A}_{2}\right) \cap \operatorname{Red}(H, t)\right]_{\approx} .
$$

Thus, there exist sequences $s_{1}$ and $s_{2}$ such that

$$
s \approx s_{1} \in \mathrm{L}\left(\mathcal{A}_{1}\right) \cap \operatorname{Red}(H, t) \text { and } s \approx s_{2} \in \mathrm{L}\left(\mathcal{A}_{2}\right) \cap \operatorname{Red}(H, t) .
$$

Hence, $s_{1} \approx s_{2}$ and Lemma 5 implies $s_{1} \sim s_{2} \in \mathrm{L}\left(\mathcal{A}_{2}\right)$. Since $\mathcal{A}_{2}$ is $\sim$-saturated, we have $s_{1} \in \mathrm{L}\left(\mathcal{A}_{2}\right)$. Thus,

$$
s \approx s_{1} \in \mathrm{L}\left(\mathcal{A}_{1}\right) \cap \mathrm{L}\left(\mathcal{A}_{2}\right) \cap \operatorname{Red}(H, t),
$$

i.e., $s \in\left[\mathrm{L}\left(\mathcal{A}_{1}\right) \cap \mathrm{L}\left(\mathcal{A}_{2}\right) \cap \operatorname{Red}(H, t)\right] \approx$.

The next lemma is straightforward to prove.

Lemma 18. Let $\mathcal{A}_{1}$ and $\mathcal{A}_{2}$ be partitioned fta. Then we have:

(a) If $\mathcal{A}_{1}$ and $\mathcal{A}_{2}$ are both deterministic, then $\mathcal{A}_{1} \times \mathcal{A}_{2}$ is deterministic, too.

(b) If $\mathcal{A}_{1}$ and $\mathcal{A}_{2}$ are both complete, then $\mathcal{A}_{1} \times \mathcal{A}_{2}$ is complete, too.

Definition 19 (complement automaton). For a partitioned, deterministic, and complete fta $\mathcal{A}=\langle\mathcal{L}, \mathrm{Q}, \tau, \delta, \mathrm{I}, \mathrm{T}\rangle$ we define the complement $\mathrm{fta} \overline{\mathcal{A}}=$ $\left\langle\mathcal{L}, \mathrm{Q}, \tau, \delta, \mathrm{I}, \tau^{-1}(1,1) \backslash \mathrm{T}\right\rangle \times \mathcal{R}_{6}$. It is again a partitioned, deterministic, and complete fta (by Lemma 18 and the fact that $\mathcal{R}_{6}$ is deterministic and complete). 
Lemma 20. Let $\mathcal{A}$ be a partitioned, deterministic, and complete fta. Then $\mathrm{L}(\overline{\mathcal{A}})=$ $\operatorname{Red}(H, t) \backslash \mathrm{L}(\mathcal{A})$. Moreover, if $\mathcal{A}$ is $\sim$-saturated, then $\overline{\mathcal{A}}$ is again $\sim$-saturated.

Proof. For the first statement, note that $\mathrm{L}(\overline{\mathcal{A}}) \subseteq \operatorname{Red}(H, t)$ since we take the product with $\mathcal{R}_{6}$. Moreover, for a partitioned, deterministic, and complete fta $\mathcal{A}$, there exists for every reduced sequence $s \in \operatorname{Red}(H, t)$ exactly one state $q \in \tau_{\mathcal{A}}^{-1}(1,1)$ such that $\left(q_{0}, q\right) \in \mu_{\mathcal{A}}(s)$, where $q_{0}$ is the unique initial state of $\mathcal{A}$. This implies $\mathrm{L}(\overline{\mathcal{A}})=\operatorname{Red}(H, t) \backslash \mathrm{L}(\mathcal{A})$.

For the second statement, note that $\left\langle\mathcal{L}, \mathrm{Q}, \tau, \delta, \mathrm{I}, \tau^{-1}(1,1) \backslash \mathrm{T}\right\rangle$ is $\sim$-saturated, since this automaton has the same $\mu$-mapping as the $\sim$-saturated fta $\mathcal{A}$. Hence, $\overline{\mathcal{A}}$ is $\sim$-saturated by Lemma 17 and the fact that $\mathcal{R}_{6}$ is $\sim$-saturated.

\subsection{Automata and morphisms}

Let us consider another HNN-extension $G^{\prime}$ defined by an homomorphism $\pi^{\prime}: H \rightarrow$ $H^{\prime}$ satisfying (19) as described in Section 3.1 by (20). Given an fta $\mathcal{A}=\langle\mathcal{L}, \mathrm{Q}, \delta, \mathrm{I}, \mathrm{T}\rangle$, we define

$$
\pi_{*}^{\prime}(\mathcal{A})=\left\langle\mathcal{L}^{\prime}, \mathrm{Q}, \delta^{\prime}, \mathrm{I}, \mathrm{T}\right\rangle
$$

with $\mathcal{L}^{\prime}=\left\{\pi^{\prime}(L) \mid L \in \mathcal{L}\right\}$ and

$$
\delta^{\prime}=\left\{\left(q, \pi^{\prime}(L), r\right) \in \mathrm{Q} \times \mathcal{L}^{\prime} \times \mathrm{Q} \mid(q, L, r) \in \delta\right\} .
$$

We denote the congruence on $H^{\prime} *\left\{t, t^{-1}\right\}$, which is defined in the same way as $\sim$ on $H *\left\{t, t^{-1}\right\}$, with $\sim^{\prime}$.

Lemma 21. Let $\mathcal{A}$ be some fta. Then we have:

(a) $\mathrm{L}\left(\pi_{*}^{\prime}(\mathcal{A})\right)=\pi_{*}^{\prime}(\mathrm{L}(\mathcal{A}))$

(b) If $\mathcal{A}$ is $\sim$-saturated, then $\pi_{*}^{\prime}(\mathcal{A})$ is $\sim^{\prime}$-saturated.

Proof. Statement (a) is obvious. For statement (b) assume that $\mathcal{A}$ is $\sim$-saturated and that

$$
q_{1}{\stackrel{h_{1}}{\longrightarrow}}_{\pi_{*}^{\prime}(\mathcal{A})} q_{2} \stackrel{t}{\rightarrow}_{\pi_{*}^{\prime}(\mathcal{A})} q_{3}{\stackrel{h_{2}}{\longrightarrow}}_{\pi_{*}^{\prime}(\mathcal{A})} q_{4} .
$$

Hence, there exist $k_{1}, k_{2} \in H$ with $h_{1}=\pi^{\prime}\left(k_{1}\right), h_{2}=\pi^{\prime}\left(k_{2}\right)$, and

$$
q_{1} \stackrel{k_{1}}{\longrightarrow} q_{2} \stackrel{t}{\rightarrow} \mathcal{A} q_{3} \stackrel{k_{2}}{\longrightarrow} \mathcal{A} q_{4}
$$

Let $a \in A$. Since $\mathcal{A}$ is $\sim$-saturated, there are states $p_{2}, p_{3} \in \mathrm{Q}$ such that

$$
q_{1} \stackrel{k_{1} a^{-1}}{\longrightarrow} \mathcal{A} p_{2} \stackrel{t}{\rightarrow} \mathcal{A} p_{3} \stackrel{\varphi(a) k_{2}}{\longrightarrow} \mathcal{A} q_{4}
$$

and hence

$$
q_{1} \stackrel{\pi^{\prime}\left(k_{1} a^{-1}\right)}{\longrightarrow} \pi_{*}^{\prime}(\mathcal{A}) p_{2} \stackrel{t}{\rightarrow}_{\pi_{*}^{\prime}(\mathcal{A})} p_{3} \stackrel{\pi^{\prime}\left(\varphi(a) k_{2}\right)}{\longrightarrow} \pi_{*}^{\prime}(\mathcal{A}) q_{4} .
$$


Since $\pi^{\prime}(c)=c$ for all $c \in A \cup B$, we obtain

$$
q_{1} \stackrel{h_{1} a^{-1}}{\longrightarrow} \pi_{*}^{\prime}(\mathcal{A}) p_{2} \stackrel{t}{\rightarrow} \pi_{*}^{\prime}(\mathcal{A}) p_{3} \stackrel{\varphi(a) h_{2}}{\longrightarrow} \pi_{*}^{\prime}(\mathcal{A}) q_{4} .
$$

An analogous argument for $t^{-1}$ shows that $\pi_{*}^{\prime}(\mathcal{A})$ is $\sim^{\prime}$-saturated.

\subsection{Normalization of automata}

The following proposition is crucial for our further considerations.

Proposition 22. From a given partitioned and $\approx$-compatible fta $\mathcal{A}$ with labelling set $\mathcal{F} \subseteq \mathcal{P}(H)$ one can effectively construct a partitioned, $\approx$-compatible, $\sim$-saturated, and unitary fta $\mathcal{B}$ with labelling set $\left\{c F c^{\prime} \mid F \in \mathcal{F}, c, c^{\prime} \in A \cup B\right\}$ such that $[\mathrm{L}(\mathcal{A})]_{\approx} \cap$ $\operatorname{Red}(H, t)=\mathrm{L}(\mathcal{B}) \cap \operatorname{Red}(H, t)$.

Proof. Let $\mathcal{A}=\langle\mathcal{L}, \mathrm{Q}, \tau, \delta, \mathrm{I}, \mathrm{T}\rangle$ be some partitioned and $\approx$-compatible fta with labeling set $\mathcal{F} \subseteq \mathcal{P}(H)$. Thus, we have

$$
[\mathrm{L}(\mathcal{A})]_{\approx}=[\mathrm{L}(\mathcal{A}) \cap \operatorname{Red}(H, t)]_{\approx} .
$$

Since $\mathcal{A}$ is partitioned, Lemma 7 implies that we can replace the set of transitions $\delta$ by the set

$$
\{(p, L, q) \in \delta \mid \tau(p) \neq \tau(q)\} \cup\{(q, 1, q) \mid q \in \mathrm{Q}\}
$$

and preserve the recognized language. The second term of the above union ensures that property (23) still holds. Such a modification might modify the mappings $\mu_{\mathcal{A}, 0}$ and $\mu_{\mathcal{A}}$, but for every sequence $s$ it does not modify the relation $\mu_{\mathcal{A}}(s) \cap \tau^{-1}(1, H) \times$ $\tau^{-1}(1,1)$, i.e., after this modification, $\mathcal{A}$ is still a partitioned and $\approx$-compatible fta. Hence, w.l.o.g. we assume that

$$
\forall(p, L, q) \in \delta: \tau(p) \neq \tau(q) \vee(p=q \wedge L=\{1\}) .
$$

Note that property (38) implies that $\mathcal{A}$ is unitary according to Definition 11.

We define the fta $\mathcal{B}=\left\langle\mathcal{L}_{\mathcal{B}}, Q_{\mathcal{B}}, \tau_{\mathcal{B}}, \delta_{\mathcal{B}}, I, T\right\rangle$ in the following way: Let us denote for every $q \in \mathrm{Q}$ by $\gamma(q)$ the subgroup

$$
\gamma(q)=\pi_{1}(\tau(q)) \in\{1, A, B\}
$$

where $\pi_{1}$ denotes the projection onto the first component of a type. Since $\mathcal{A}$ is partitioned, if $(q, t, r) \in \delta$ (resp. $\left.\left(q, t^{-1}, r\right) \in \delta\right)$ then $\gamma(q)=A$ (resp. $\left.\gamma(r)=B\right)$ and $\gamma(q)=B($ resp. $\gamma(r)=A)$. We set

$$
\mathrm{Q}_{\mathcal{B}}=\{(q, g) \in \mathrm{Q} \times(A \cup B) \mid g \in \gamma(q)\} .
$$

We shall identify $(q, 1)$ with $q$ and denote $(q, g)$ by $q g$ in the sequel. Note that the set of initial (resp. terminal) states of $\mathcal{B}$ is the same as for $\mathcal{A}$. The typing map $\tau_{\mathcal{B}}$ is inherited from $\mathcal{A}$ :

$$
\tau_{\mathcal{B}}(q g)=\tau(q)
$$



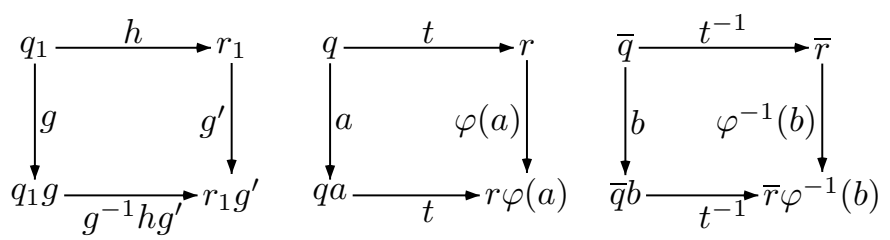

Fig. 4. Some subgraphs of $\mathcal{B}$

The set $\delta_{\mathcal{B}}$ consists of all the following transitions, where $q, r \in \mathrm{Q}$ :

$$
\begin{gathered}
\left(q g, g^{\prime}, q\left(g g^{\prime}\right)\right) \text { for } g, g^{\prime} \in \gamma(q) \\
\left(q g, g^{-1} L g^{\prime}, r g^{\prime}\right) \text { for } g \in \gamma(q), g^{\prime} \in \gamma(r), L \subseteq H,(q, L, r) \in \delta \\
\quad(q a, t, r \varphi(a)) \text { for } a \in A,(q, t, r) \in \delta \\
\left(q b, t^{-1}, r \varphi^{-1}(b)\right) \text { for } b \in B,\left(q, t^{-1}, r\right) \in \delta
\end{gathered}
$$

For all $\left(q_{1}, L, r_{1}\right),(q, t, r),\left(\bar{q}, t^{-1}, \bar{r}\right) \in \delta, g \in \gamma\left(q_{1}\right), g^{\prime} \in \gamma\left(r_{1}\right), h \in L, a \in A$, and $b \in B$, the automaton $\mathcal{B}$ contains the three basic subgraphs exhibited in Figure 4. We cut the proof that $\mathcal{B}$ has the required properties into five claims.

Claim 23. $[\mathrm{L}(\mathcal{A})]_{\approx} \cap \operatorname{Red}(H, t)=[\mathrm{L}(\mathcal{A}) \cap \operatorname{Red}(H, t)]_{\sim}$

By intersecting both sides of $(37)$ with $\operatorname{Red}(H, t)$, we obtain

$$
[\mathrm{L}(\mathcal{A})]_{\approx} \cap \operatorname{Red}(H, t)=[\mathrm{L}(\mathcal{A}) \cap \operatorname{Red}(H, t)]_{\approx} \cap \operatorname{Red}(H, t) .
$$

But by Lemma 5 , the right-hand side above equals $[\mathrm{L}(\mathcal{A}) \cap \operatorname{Red}(H, t)]_{\sim}$, which proves Claim 23.

Claim 24. $\mathrm{L}(\mathcal{A}) \subseteq \mathrm{L}(\mathcal{B})$

Let us notice that every $(q, L, r) \in \delta$ has a copy in $\delta_{\mathcal{B}}$ under the form of $(q 1, L, r 1)$, since whatever $\gamma(q)$ and $\gamma(r)$ are, $1 \in \gamma(q) \cap \gamma(r)$. Thus, up to the identification of every state $q \in \mathrm{Q}$ with the state $q 1 \in \mathrm{Q}_{\mathcal{B}}, \delta \subseteq \delta_{\mathcal{B}}$. Since moreover $\mathrm{I}=\mathrm{I}_{\mathcal{B}}$ and $\mathrm{T}=\mathrm{T}_{\mathcal{B}}$, we obtain Claim 24 .

Claim 25. The fta $\mathcal{B}$ is $\sim$-saturated and hence, $\mathrm{L}(\mathcal{B})=[\mathrm{L}(\mathcal{B})]_{\sim}$.

Let us assume that

$$
q_{0} a_{0} \stackrel{h}{\rightarrow} \mathcal{B} q_{1} a_{1} \stackrel{t}{\rightarrow}_{\mathcal{B}} q_{2} a_{2} \stackrel{h^{\prime}}{\rightarrow} \mathcal{B} q_{3} a_{3},
$$

where $q_{0}, \ldots, q_{3} \in \mathrm{Q}, a_{i} \in \gamma\left(q_{i}\right)$, and $h, h^{\prime} \in H$. Thus, $\left(q_{1}, t, q_{2}\right) \in \delta$ and $a_{2}=\varphi\left(a_{1}\right)$. Moreover, $\gamma\left(q_{1}\right)=A$, since a $t$-transition is enabled in $q_{1} a_{1}$. The transition $q_{0} a_{0} \stackrel{h}{\rightarrow} \mathcal{B}$ $q_{1} a_{1}$ is either of type (39) or (40). In both cases, we can derive for every $a \in A$ the transition

$$
q_{0} a_{0} \stackrel{h a^{-1}}{\longrightarrow} \mathcal{B} q_{1}\left(a_{1} a^{-1}\right) .
$$

Analogously, we get

$$
q_{2} \varphi\left(a_{1} a^{-1}\right) \stackrel{\varphi(a) h^{\prime}}{\longrightarrow} \mathcal{B} q_{3} a_{3} .
$$


Finally, $q_{1} a_{1} \stackrel{t}{\rightarrow} \mathcal{B} q_{2} \varphi\left(a_{1}\right)$ and (41) implies

$$
q_{1}\left(a_{1} a^{-1}\right) \stackrel{t}{\rightarrow} \mathcal{B} q_{2} \varphi\left(a_{1} a^{-1}\right) .
$$

Altogether, it follows

$$
q_{0} a_{0} \stackrel{\left(h a^{-1}\right) t\left(\varphi(a) h^{\prime}\right)}{\longrightarrow}{ }_{\mathcal{B}} q_{3} a_{3} .
$$

Similarly, if $q_{0} a_{0} \stackrel{h t^{-1} h^{\prime}}{\longrightarrow} q_{3} a_{3}$ and $b \in B$, then

$$
q_{0} a_{0} \stackrel{\left(h b^{-1}\right) t^{-1}\left(\varphi^{-1}(b) h^{\prime}\right)}{\longrightarrow}{ }_{\mathcal{B}} q_{3} a_{3} .
$$

This proves Claim 25.

Claim 26. $\mathrm{L}(\mathcal{B}) \subseteq[\mathrm{L}(\mathcal{A})]_{\sim}$

Let $s \in H *\left\{t, t^{-1}\right\}^{*}, q, r \in \mathrm{Q}, g \in \gamma(q)$, and $h \in \gamma(r)$ such that

$$
q g \stackrel{s}{\rightarrow}_{\mathcal{B}} r h .
$$

By gluing squares from Figure 4 along their vertical edges, one can prove by induction on the integer $n$ of the decomposition (15) that, under the hypothesis (46), there exists $s^{\prime} \sim g s h^{-1}$ such that

$$
q \stackrel{s^{\prime}}{\rightarrow} \mathcal{A} r .
$$

If $q \in \mathrm{I}$ and $r \in \mathrm{T}$, then the type-conditions (30) imply $\gamma(q)=\gamma(r)=1$, i.e., $g=h=1$ and $s \in \mathrm{L}(\mathcal{B})$. Then (47) says that

$$
\exists s^{\prime}: s^{\prime} \sim s \wedge s^{\prime} \in \mathrm{L}(\mathcal{A}),
$$

which proves Claim 26.

Claim 27. $\mathrm{L}(\mathcal{B}) \subseteq[\mathrm{L}(\mathcal{B}) \cap \operatorname{Red}(H, t)] \approx$

Claim 27 can be deduced as follows:

$$
\begin{aligned}
\mathrm{L}(\mathcal{B}) & \subseteq[\mathrm{L}(\mathcal{A})]_{\sim} & & (\text { by Claim } 26) \\
& \subseteq[\mathrm{L}(\mathcal{A})]_{\approx} & & (\text { since } \sim \subseteq \approx) \\
& =[\mathrm{L}(\mathcal{A}) \cap \operatorname{Red}(H, t)]_{\approx} & & (\text { since } \mathcal{A} \text { is } \approx \text {-compatible }) \\
& \subseteq[\mathrm{L}(\mathcal{B}) \cap \operatorname{Red}(H, t)]_{\approx} & & (\text { by Claim 24) }
\end{aligned}
$$

Let us now use Claim 23-27 in order to show point Proposition 22: First, we have

$$
\begin{aligned}
{[\mathrm{L}(\mathcal{A})]_{\approx} \cap \operatorname{Red}(H, t) } & =[\mathrm{L}(\mathcal{A}) \cap \operatorname{Red}(H, t)]_{\sim} & & (\text { by Claim 23) } \\
& \subseteq[\mathrm{L}(\mathcal{A})]_{\sim} & & \\
& \subseteq[\mathrm{L}(\mathcal{B})]_{\sim} & & \text { (by Claim 24) } \\
& =\mathrm{L}(\mathcal{B}) & & \text { (by Claim 25), }
\end{aligned}
$$

and thus

$$
[\mathrm{L}(\mathcal{A})] \approx \cap \operatorname{Red}(H, t) \subseteq \mathrm{L}(\mathcal{B}) \cap \operatorname{Red}(H, t) .
$$


On the other hand, we have

$$
\begin{aligned}
\mathrm{L}(\mathcal{B}) \cap \operatorname{Red}(H, t) & \subseteq[\mathrm{L}(\mathcal{A})]_{\sim} \cap \operatorname{Red}(H, t) \quad(\text { by Claim } 26) \\
& \subseteq[\mathrm{L}(\mathcal{A})]_{\approx} \cap \operatorname{Red}(H, t) \quad(\text { since } \sim \subseteq \approx) .
\end{aligned}
$$

Hence, we obtain

$$
[\mathrm{L}(\mathcal{A})]_{\approx} \cap \operatorname{Red}(H, t)=\mathrm{L}(\mathcal{B}) \cap \operatorname{Red}(H, t),
$$

as was required. Moreover:

- $\mathcal{B}$ is partitioned, since $\mathcal{A}$ is partitioned.

- By Claim $27, \mathcal{B}$ is $\approx$-compatible.

- By Claim $25, \mathcal{B}$ is $\sim$-saturated.

- $\mathcal{B}$ is unitary: If $g^{\prime}=1$ in (39), then we have $q g=q g g^{\prime}$. If $1 \in g^{-1} L g^{\prime}$ and $(q, L, r) \in \delta$ in (40), then property (38) guarantees that $\tau(q) \neq \tau(r)$ or $(q=r$ and $L=\{1\})$. If $\tau(q) \neq \tau(r)$ then $\tau_{\mathcal{B}}(q g) \neq \tau_{\mathcal{B}}\left(r g^{\prime}\right)$. If $q=r$ and $L=\{1\}$, then $g=g^{\prime}$ and hence $q g=r g^{\prime}$. Hence, no $\mathcal{B}$-transition of type (39) or (40) has the form $\left(q g, L, r g^{\prime}\right)$ with $\tau_{\mathcal{B}}(q g)=\tau_{\mathcal{B}}\left(r g^{\prime}\right), 1 \in L$, and $q g \neq r g^{\prime}$.

Proposition 22 is thus established.

Recall the definition of a deterministic (Definition 12) and complete (Definition 13) fta.

Proposition 28. From a given partitioned, $\approx$-compatible, and $\sim$-saturated fta $\mathcal{B}$ with labelling set $\mathcal{F} \subseteq \mathcal{P}(H)$ one can effectively construct a partitioned, saturated, deterministic, and complete fta $\mathcal{C}$ with labelling set $\operatorname{Bool}(\mathcal{F})$ such that $\mathrm{L}(\mathcal{B}) \cap \operatorname{Red}(H, t)=\mathrm{L}(\mathcal{C})$.

Proof. The proof is essentially a variant of the standard power set construction for determinizing ordinary finite state automata over words. Let $\mathcal{B}=$ $\left\langle\mathcal{L}_{\mathcal{B}}, Q_{\mathcal{B}}, \tau_{\mathcal{B}}, \delta_{\mathcal{B}}, I_{\mathcal{B}}, T_{\mathcal{B}}\right\rangle$ be a partitioned, $\approx$-compatible, and $\sim$-saturated fta. We first define a partitioned fta

$$
\mathcal{D}=\left\langle\mathcal{L}_{\mathcal{D}}, Q_{\mathcal{D}}, \tau_{\mathcal{D}}, \delta_{\mathcal{D}}, I_{\mathcal{D}}, T_{\mathcal{D}}\right\rangle
$$

in the following way: Let

$$
\mathcal{L}^{\prime}=\left(\mathcal{L}_{\mathcal{B}} \cap \mathcal{P}(H)\right) \cup\{\{c\} \mid c \in A \cup B\}
$$

and

$$
\mathcal{L}_{\mathcal{D}}=\left\{\{t\},\left\{t^{-1}\right\}\right\} \cup\left\{\bigcap_{L \in \mathcal{K}} L \cap \bigcap_{L \in \mathcal{L}^{\prime} \backslash \mathcal{K}} H \backslash L \mid \mathcal{K} \subseteq \mathcal{L}^{\prime}\right\} \backslash\{\emptyset\} .
$$

Thus, $\mathcal{L}_{\mathcal{D}} \cap \mathcal{P}(H)$ is the set of atoms of the boolean algebra generated by $\mathcal{L}^{\prime}$. Let

$$
\mathrm{Q}_{\mathcal{D}}=\left\{(P, \Theta) \mid P \subseteq \mathrm{Q}_{\mathcal{B}}, \Theta \in \mathcal{T}_{6}, P \subseteq \tau_{\mathcal{B}}^{-1}(\Theta)\right\}
$$


The type function is the projection onto the second component:

$$
\tau_{\mathcal{D}}(P, \Theta)=\Theta .
$$

The set of transitions $\delta_{\mathcal{D}}$ consists of all triples $\left((P, \Theta), K,\left(P^{\prime}, \Theta^{\prime}\right)\right) \in \mathrm{Q}_{\mathcal{D}} \times \mathcal{L}_{\mathcal{D}} \times \mathrm{Q}_{\mathcal{D}}$ such that

$$
\begin{aligned}
& P^{\prime}=\left\{p^{\prime} \in Q_{\mathcal{B}} \mid \exists p \in P \exists L:\left(p, L, p^{\prime}\right) \in \delta_{\mathcal{B}}, K \subseteq L\right\} \cap \tau_{\mathcal{B}}^{-1}\left(\Theta^{\prime}\right) \\
& \text { and }\{\Theta\} \times K \times\left\{\Theta^{\prime}\right\} \subseteq \widehat{\mathcal{E}}_{6},
\end{aligned}
$$

where $\mathcal{E}_{6}$ is the edge relation of the graph $\mathcal{G}_{6}$ and $\widehat{\mathcal{E}}_{6}$ is defined by $(24)$. Note that $P^{\prime}=\emptyset$ is allowed. Finally, let

$$
\mathbf{I}_{\mathcal{D}}=\left\{\left(\mathrm{I}_{\mathcal{B}},(1, H)\right)\right\} \text { and } \mathbf{T}_{\mathcal{D}}=\left\{(P,(1,1)) \in \mathbf{Q}_{\mathcal{D}} \mid P \cap \mathbf{T}_{\mathcal{B}} \neq \emptyset\right\} .
$$

The definition of $\delta_{\mathcal{D}}$ implies that $\mathcal{D}$ is partitioned and complete. Moreover, the fta $\mathcal{D}$ is deterministic: if we have two transitions $\left((P, \Theta), K_{i},\left(P_{i}, \Theta^{\prime}\right)\right) \in \delta_{\mathcal{D}}(i \in\{1,2\})$ with $K_{1} \cap K_{2} \neq \emptyset$ then we must have $K_{1}=K_{2}$ and hence $P_{1}=P_{2}$.

Remark 29. For every $P \subseteq Q_{\mathcal{B}}$ fulfilling $\emptyset \subsetneq P \subseteq \tau^{-1}(\Theta),(P, \Theta)$ is a state of $\mathcal{D}$ and no pair $\left(P, \Theta^{\prime}\right)$ with $\Theta \neq \Theta^{\prime}$ is a state of $\mathcal{D}$. But $(\emptyset, \Theta)$ is a state of $\mathcal{D}$ for every type $\Theta$. This is in some sense the price to pay for the completeness of $\mathcal{D}$.

Recall the definition of the mapping $\mu_{\mathcal{B}, 0}$ from (25).

Claim 30. Let $(P, \Theta),\left(P^{\prime}, \Theta^{\prime}\right) \in \mathrm{Q}_{\mathcal{D}}$ and $s \in H \cup\left\{t, t^{-1}\right\}$. Then

$$
\left((P, \Theta),\left(P^{\prime}, \Theta^{\prime}\right)\right) \in \mu_{\mathcal{D}, 0}(s) \Leftrightarrow P^{\prime}=\left(P \odot \mu_{\mathcal{B}, 0}(s)\right) \cap \tau_{\mathcal{B}}^{-1}\left(\Theta^{\prime}\right) \wedge\left(\Theta, s, \Theta^{\prime}\right) \in \widehat{\mathcal{E}}_{6} .
$$

Remark 31. If $P^{\prime}=\left(P \odot \mu_{\mathcal{B}, 0}(s)\right) \cap \tau_{\mathcal{B}}^{-1}\left(\Theta^{\prime}\right) \neq \emptyset$, then the condition $\left(\Theta, s, \Theta^{\prime}\right) \in \widehat{\mathcal{E}}_{6}$ follows automatically, since the fta $\mathcal{B}$ is partitioned. But since $P^{\prime}=\emptyset$ is possible, we have to add the condition $\left(\Theta, s, \Theta^{\prime}\right) \in \widehat{\mathcal{E}}_{6}$ explicitly.

In order to prove Claim 30, we distinguish three cases.

Case 1: $s=t$. Note that $((A, T), t,(B, H))$ is the only $t$-labelled edge of $\mathcal{G}_{6}$. We get:

$$
\begin{aligned}
& \left((P, \Theta),\left(P^{\prime}, \Theta^{\prime}\right)\right) \in \mu_{\mathcal{D}, 0}(t) \\
\Leftrightarrow & \left((P, \Theta), t,\left(P^{\prime}, \Theta^{\prime}\right)\right) \in \delta_{\mathcal{D}} \\
\Leftrightarrow & P^{\prime}=P \odot \mu_{\mathcal{B}, 0}(t) \cap \tau_{\mathcal{B}}^{-1}\left(\Theta^{\prime}\right) \wedge \Theta=(A, T) \wedge \Theta^{\prime}=(B, H) .
\end{aligned}
$$

Case 2: $s=t^{-1}$. Same kind of arguments as for $s=t$.

Case 3: $s=h \in H$. Let $K(h)$ be the unique atom of $\mathcal{L}_{\mathcal{D}} \cap \mathcal{P}(H)$ such that $h \in K(h)$. For every transition $(p, L, q) \in \delta_{\mathcal{B}}$, one has

$$
h \in L \Leftrightarrow K(h) \subseteq L .
$$


It follows that

$$
\begin{aligned}
\left((P, \Theta),\left(P^{\prime}, \Theta^{\prime}\right)\right) \in \mu_{\mathcal{D}, 0}(h) \Leftrightarrow & \left((P, \Theta), K(h),\left(P^{\prime}, \Theta^{\prime}\right)\right) \in \delta_{D} \\
\Leftrightarrow & P^{\prime}=\left(P \odot \mu_{\mathcal{B}, 0}(h)\right) \cap \tau_{\mathcal{B}}^{-1}\left(\Theta^{\prime}\right) \text { and } \\
& \{\Theta\} \times K(h) \times\left\{\Theta^{\prime}\right\} \subseteq \widehat{\mathcal{E}}_{6} \\
\Leftrightarrow \quad & P^{\prime}=\left(P \odot \mu_{\mathcal{B}, 0}(h)\right) \cap \tau_{\mathcal{B}}^{-1}\left(\Theta^{\prime}\right) \text { and } \\
& \left(\Theta, h, \Theta^{\prime}\right) \in \widehat{\mathcal{E}}_{6} .
\end{aligned}
$$

For the last equivalence note that $\{\Theta\} \times K(h) \times\left\{\Theta^{\prime}\right\} \subseteq \widehat{\mathcal{E}}_{6}$ if and only if $\left(\Theta, h, \Theta^{\prime}\right) \in$ $\widehat{\mathcal{E}}_{6}$. For this, it is important that if $h \in A \cup B$ then $K(h)=\{h\}$; note that every singleton $\{c\}$ with $c \in A \cup B$ belongs to $\mathcal{L}^{\prime}$ in (49).

Claim 32. Let $(P, \Theta),\left(P^{\prime}, \Theta^{\prime}\right) \in \mathrm{Q}_{\mathcal{D}}$ and $s \in H *\left\{t, t^{-1}\right\}^{*}$. Then

$$
\left((P, \Theta),\left(P^{\prime}, \Theta^{\prime}\right)\right) \in \mu_{\mathcal{D}}(s) \Leftrightarrow P^{\prime}=\left(P \odot \mu_{\mathcal{B}}(s)\right) \cap \tau_{\mathcal{B}}^{-1}\left(\Theta^{\prime}\right) \wedge\left(\Theta, \Theta^{\prime}\right) \in \mu_{\mathcal{G}_{6}}(s) .
$$

Let us prove this claim by induction over the integer $n$ appearing in decomposition (15) of the $t$-sequence $s$.

Base case: $n=0$. For $s=h \in H$ the assertion of Claim 32 is equivalent with that of Claim 30, since $\mu_{\mathcal{A}}(h)=\mu_{\mathcal{A}, 0}(h)$ for every fta $\mathcal{A}$.

Induction step: Let $s$ be of the form (15) with $n \geq 1$. We suppose that $\alpha_{n}=1$ and let

$$
s_{1}=h_{0} t^{\alpha_{1}} h_{1} \cdots t^{\alpha_{i}} h_{i} \cdots t^{\alpha_{n-1}} h_{n-1} \text { and } s=s_{1} t h_{n}
$$

(where $s_{1}=h_{0}$ if $n=1$ ). Let us first assume that

$$
\left((P, \Theta),\left(P^{\prime}, \Theta^{\prime}\right)\right) \in \mu_{\mathcal{D}}(s) .
$$

The definition of $\delta_{\mathcal{D}}$ implies $\left(\Theta, \Theta^{\prime}\right) \in \mu_{\mathcal{G}_{6}}(s)$. Moreover, the structure of the graph $\mathcal{G}_{6}$ implies that there exist $P_{1} \subseteq \tau_{\mathcal{B}}^{-1}(A, T)$ and $P_{2} \subseteq \tau_{\mathcal{B}}^{-1}(B, H)$ with

$$
\begin{aligned}
\left((P, \Theta),\left(P_{1},(A, T)\right)\right) & \in \mu_{\mathcal{D}}\left(s_{1}\right), \\
\left(\left(P_{1},(A, T)\right),\left(P_{2},(B, H)\right)\right) & \in \mu_{\mathcal{D}, 0}(t), \text { and } \\
\left(\left(P_{2},(B, H)\right),\left(P^{\prime}, \Theta^{\prime}\right)\right) & \in \mu_{\mathcal{D}, 0}\left(h_{n}\right) .
\end{aligned}
$$

As Claim 32 is fulfilled by $s_{1}$ (by the induction hypothesis) and also by sequences in $H \cup\left\{t, t^{-1}\right\}$ (by Claim 30), equations (51)-(53) imply:

$$
\begin{aligned}
\left(P \odot \mu_{\mathcal{B}}\left(s_{1}\right)\right) \cap \tau_{\mathcal{B}}^{-1}(A, T) & =P_{1} \\
\left(P_{1} \odot \mu_{\mathcal{B}, 0}(t)\right) \cap \tau_{\mathcal{B}}^{-1}(B, H) & =P_{2} \\
\left(P_{2} \odot \mu_{\mathcal{B}, 0}\left(h_{n}\right)\right) \cap \tau_{\mathcal{B}}^{-1}\left(\Theta^{\prime}\right) & =P^{\prime}
\end{aligned}
$$

Omitting the type intersections in (54) and (55), we obtain

$$
\left(P \odot \mu_{\mathcal{B}}\left(s_{1} t h_{n}\right)\right) \cap \tau_{\mathcal{B}}^{-1}(\Theta) \supseteq P^{\prime} .
$$


Conversely, by the structure of $\mathcal{G}_{6}$, every path in the partitioned fta $\mathcal{B}$ from a state $p \in P$ to a state $q^{\prime} \in \tau_{\mathcal{B}}^{-1}\left(\Theta^{\prime}\right)$, reading $s_{1} t h_{n}$, must decompose into

$$
p \stackrel{s_{1}}{\longrightarrow} \mathcal{B} p_{1} \stackrel{t}{\rightarrow} \mathcal{B} p_{2} \stackrel{h_{n}}{\longrightarrow} \mathcal{B} q^{\prime}
$$

with $\tau_{\mathcal{B}}\left(p_{1}\right)=(A, T)$ and $\tau_{\mathcal{B}}\left(p_{2}\right)=(B, H)$. Hence, $(54)-(56)$ imply

$$
\left(P \odot \mu_{\mathcal{B}}\left(s_{1} t h_{n}\right)\right) \cap \tau_{\mathcal{B}}^{-1}\left(\Theta^{\prime}\right) \subseteq P^{\prime} .
$$

From (57) and (58) we obtain $P \odot \mu_{\mathcal{B}}\left(s_{1} t h_{n}\right) \cap \tau_{\mathcal{B}}^{-1}\left(\Theta^{\prime}\right)=P^{\prime}$. This proves implication $\Rightarrow$ from Claim 32 .

Now, suppose conversely that $P \odot \mu_{\mathcal{B}}\left(s_{1} t h_{n}\right) \cap \tau_{\mathcal{B}}^{-1}\left(\Theta^{\prime}\right)=P^{\prime}$ and $\left(\Theta, \Theta^{\prime}\right) \in$ $\mu_{\mathcal{G}_{6}}(s)$. By the same kind of arguments as above, there must exist $P_{1} \subseteq \tau_{\mathcal{B}}^{-1}(A, T)$ and $P_{2} \subseteq \tau_{\mathcal{B}}^{-1}(B, H)$ fulfilling equations (54)-(56). Moreover, from $\left(\Theta, \Theta^{\prime}\right) \in \mu_{\mathcal{G}_{6}}(s)$ we get $(\Theta,(A, T)) \in \mu_{\mathcal{G}_{6}}\left(s_{1}\right)$ and $\left((B, H), \Theta^{\prime}\right) \in \mu_{\mathcal{G}_{6}}\left(h_{n}\right)$. By the induction hypothesis and Claim 30 this leads to (51)-(53), and hence to (50).

The induction step in the case where $\alpha_{n}=-1$ can be treated analogously. This proves Claim 32.

From Claim 32 and the fact that the fta $\mathcal{B}$ and $\mathcal{G}_{6}$ are $\sim$-saturated, it follows immediately that also $\mathcal{D}$ is $\sim$-saturated. Moreover,

$$
\mathrm{L}(\mathcal{D})=\mathrm{L}(\mathcal{B}) .
$$

Let us finally take for $\mathcal{C}$ the product automaton (see Definition 14) $\mathcal{C}=\mathcal{D} \times \mathcal{R}_{6}$, where $\mathcal{R}_{6}$ is the partitioned fta from Figure 3 . The partitioned fta $\mathcal{C}$ has the following properties:

- $\mathrm{L}(\mathcal{C})=\mathrm{L}(\mathcal{D}) \cap \operatorname{Red}(H, t)=\mathrm{L}(\mathcal{B}) \cap \operatorname{Red}(H, t)$ by Lemma 15 .

- $\mathcal{C}$ is deterministic and complete by Lemma 18 and the fact that $\mathcal{R}_{6}$ and $\mathcal{D}$ are both deterministic and complete.

- $\mathcal{C}$ is $\sim$-saturated by Lemma 17 and the fact that $\mathcal{R}_{6}$ and $\mathcal{D}$ are $\sim$-saturated.

Hence, $\mathcal{C}$ fulfils Proposition 28.

Recall that $\pi:\left(X \cup\left\{t, t^{-1}\right\}\right)^{*} \rightarrow G=\left\langle H, t ; t^{-1} a t=\varphi(a)(a \in A)\right\rangle$ denotes the canonical morphism from (8), where $X$ is a generating set for $H$ with $A \cup B \subseteq X$.

Proposition 33. Let $K \subseteq G$. The following are equivalent:

(1) $K$ is a rational subset of $G$.

(2) There exists some finite automaton $\mathcal{A}$ over the free monoid $\left(X \cup\left\{t, t^{-1}\right\}\right)^{*}$ such that $K=\pi(\mathrm{L}(\mathcal{A}))$.

(3) $\pi_{G}^{-1}(K) \cap \operatorname{Red}(H, t)=\mathrm{L}(\mathcal{B}) \cap \operatorname{Red}(H, t)$ for some partitioned, $\approx$-compatible, and $\sim$-saturated fta $\mathcal{B}$ with labelling set $\operatorname{Rat}(H)$.

Moreover,

(a) in point (3), $\mathcal{B}$ can always be chosen unitary (see Definition 11), and 
(b) in point (3), if the membership problem for languages in $\operatorname{Rat}(H)$ is decidable, then $\mathcal{B}$ can be computed effectively from $\mathcal{A}$ in point (2).

Proof. The equivalence of (1) and (2) is trivial.

$(2) \Rightarrow(3)$ : We cut the proof of this implication into three claims. Let us start with a finite automaton

$$
\mathcal{A}=\langle\mathcal{X}, \mathrm{Q}, \delta, \mathrm{I}, \mathrm{T}\rangle
$$

over the free monoid $\left(X \cup\left\{t, t^{-1}\right\}\right)^{*}$ such that

$$
K=\pi(\mathrm{L}(\mathcal{A})) .
$$

Let us recall that ST is the semi-Thue system consisting of all the rules in (16) and (17), where $u \in X^{*}$ and $\varphi\left([u]_{\equiv}\right) \in B$ and $\varphi^{-1}\left([u]_{\equiv}\right) \in A$ denote the corresponding letters in $X$ (we choose such a letter for every element of the finite set $A \cup B$ ).

For a set $E$, a binary relation $\leadsto \subseteq E \times E$, and a subset $R \subseteq E$, let $R \leadsto=\{e \in$ $E \mid \exists r \in R: r \leadsto e\}$.

Claim 34. There exists a finite automaton $\mathcal{A}_{1}$ over the free monoid $\left(X \cup\left\{t, t^{-1}\right\}\right)^{*}$, such that

$$
\mathrm{L}\left(\mathcal{A}_{1}\right)=\mathrm{L}(\mathcal{A}) \rightarrow_{\mathrm{ST}}^{*} .
$$

Moreover, if the membership problem is decidable for rational subsets of $H$, then $\mathcal{A}_{1}$ can be constructed effectively from $\mathcal{A}$.

For the proof of Claim 34, notice that ST is monadic, i.e., every right-hand side of a rule has length one or zero. It is known that for every monadic semi-Thue system $S$ over an alphabet $Y$ and every rational subset $R \subseteq Y^{*}, R \rightarrow{ }_{S}^{*} \in \operatorname{Rat}\left(Y^{*}\right)$, see [BJW82,Sén94]. Taking $R=\mathrm{L}(\mathcal{A})$ and $S=\mathrm{ST}$ in this result, we obtain the first statement of Claim 34. In order to check the effectiveness statement, let us consider the following algorithm:

$1 \quad \delta_{1}:=\delta$

2 while there are $p, q, q^{\prime}, r \in \mathrm{Q}, u \in X^{*}, a \in A, b \in B$ such that

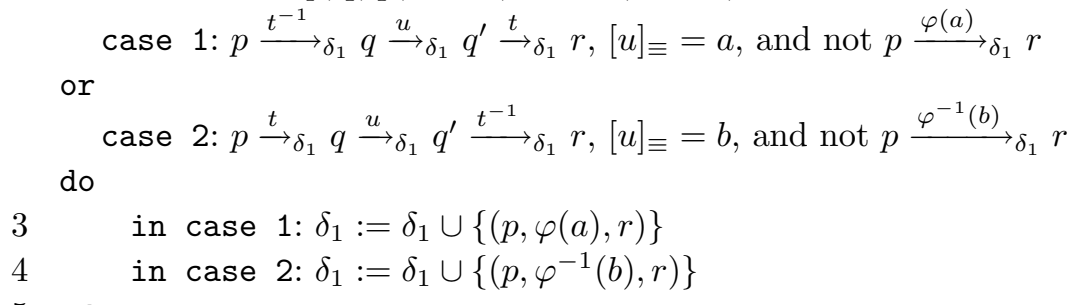

This algorithm constructs from the finite automaton $\mathcal{A}$ a finite automaton

$$
\mathcal{A}_{1}=\left\langle\mathcal{X} \cup A \cup B, \mathrm{Q}, \delta_{1}, \mathrm{I}, \mathrm{T}\right\rangle
$$


satisfying the first statement of Claim 34 . In order to make line 2 above effective, it is enough that we can decide for every $a \in A$ (resp. $b \in B$ ) and every $q, q^{\prime} \in \mathrm{Q}$, whether $a \in \pi_{t}\left(\mathrm{~L}\left(\mathcal{A}\left[X, q, q^{\prime}\right]\right)\right)\left(\right.$ resp. $\left.b \in \pi_{t}\left(\mathrm{~L}\left(\mathcal{A}\left[X, q, q^{\prime}\right]\right)\right)\right)$, where $\pi_{t}:\left(X \cup\left\{t, t^{-1}\right\}\right)^{*} \rightarrow$ $H *\left\{t, t^{-1}\right\}^{*}$ is the canonical morphism from (10) and the automaton $\mathcal{A}\left[X, q, q^{\prime}\right]$ is defined by (5). But this is an instance of the membership problem for rational subsets of $H$. This proves the effectiveness statement in Claim 34 .

Claim 35. There exists some $\approx$-compatible fta $\mathcal{A}_{2}$ over $H *\left\{t, t^{-1}\right\}^{*}$ with labelling set $\operatorname{Rat}(H)$ such that

$$
\mathrm{L}\left(\mathcal{A}_{2}\right)=\pi_{t}\left(\mathrm{~L}\left(\mathcal{A}_{1}\right)\right) .
$$

Moreover, $\mathcal{A}_{2}$ can be constructed effectively from $\mathcal{A}_{1}$.

Recall the finite automaton $\mathcal{A}_{1}$ from (59). We set

$$
\mathcal{A}_{2}=\left\langle\mathcal{L}, \mathrm{Q}, \delta_{2}, \mathrm{I}, \mathrm{T}\right\rangle
$$

where

$$
\begin{aligned}
\delta_{2}= & \left\{\left(q, t^{\alpha}, r\right) \mid \alpha \in\{1,-1\},\left(q, t^{\alpha}, r\right) \in \delta_{1}\right\} \cup \\
& \left\{\left(q, \pi_{t}\left(\mathrm{~L}\left(\mathcal{A}_{1}[X, q, r]\right)\right), r\right) \mid q, r \in \mathrm{Q}\right\}
\end{aligned}
$$

(the label set $\mathcal{L}$ is given implicitly by $\delta_{2}$ ). In some sense the transitions of $\delta_{2}$ are collecting into the rational labels $\pi_{t}\left(\mathrm{~L}\left(\mathcal{A}_{1}[X, q, r]\right)\right) \in \operatorname{Rat}(H)$ all possible finite paths with labels in $X^{*}$, while the transitions labelled by $t$ or $t^{-1}$ are just copied. Clearly, $\mathrm{L}\left(\mathcal{A}_{2}\right)=\pi_{t}\left(\mathrm{~L}\left(\mathcal{A}_{1}\right)\right)$ and $\mathcal{A}_{2}$ can be constructed effectively from $\mathcal{A}_{1}$. So it remains to show that $\mathcal{A}_{2}$ is $\approx$-compatible.

Recall that $\pi_{t}(\operatorname{Irr}(\mathrm{ST}))=\operatorname{Red}(H, t)$. Since, $\mathrm{L}\left(\mathcal{A}_{1}\right)=\mathrm{L}(\mathcal{A}) \rightarrow_{\mathrm{ST}}^{*}$, the language $\mathrm{L}\left(\mathcal{A}_{1}\right)$ is closed under the terminating rewrite relation $\rightarrow \mathrm{sT} \subseteq \equiv$. It follows

$$
\mathrm{L}\left(\mathcal{A}_{1}\right) \subseteq\left[\mathrm{L}\left(\mathcal{A}_{1}\right) \cap \operatorname{Irr}(\mathrm{ST})\right]_{\equiv} .
$$

By applying the morphism $\pi_{t}$ to this inclusion, we obtain

$$
\begin{aligned}
\mathrm{L}\left(\mathcal{A}_{2}\right) & =\pi_{t}\left(\mathrm{~L}\left(\mathcal{A}_{1}\right)\right) \\
& \subseteq \pi_{t}\left(\left[\mathrm{~L}\left(\mathcal{A}_{1}\right) \cap \operatorname{Irr}(\mathrm{ST})\right]_{\equiv}\right) \\
& =\left[\pi_{t}\left(\mathrm{~L}\left(\mathcal{A}_{1}\right) \cap \operatorname{Irr}(\mathrm{ST})\right)\right]_{\approx} \\
& \subseteq\left[\pi_{t}\left(\mathrm{~L}\left(\mathcal{A}_{1}\right)\right) \cap \pi_{t}(\operatorname{Irr}(\mathrm{ST}))\right]_{\approx} \\
& =\left[\mathrm{L}\left(\mathcal{A}_{2}\right) \cap \operatorname{Red}(H, t)\right]_{\approx},
\end{aligned}
$$

which proves that $\mathcal{A}_{2}$ is $\approx$-compatible.

Claim 36. There exists some partitioned and $\approx$-compatible fta $\mathcal{A}_{3}$ over $H *\left\{t, t^{-1}\right\}^{*}$ with labelling set $\operatorname{Rat}(H)$ such that

$$
\mathrm{L}\left(\mathcal{A}_{3}\right)=\mathrm{L}\left(\mathcal{A}_{2}\right) .
$$

Moreover, $\mathcal{A}_{3}$ can be constructed effectively from $\mathcal{A}_{2}$. 
Just set $\mathcal{A}_{3}=\mathcal{A}_{2} \times \mathcal{G}_{6}$. By Lemma 16 , we have $\mathrm{L}\left(\mathcal{A}_{3}\right)=\mathrm{L}\left(\mathcal{A}_{2}\right)$. Hence, since $\mathcal{A}_{2}$ is $\approx$-compatible, also $\mathcal{A}_{3}$ is $\approx$-compatible.

By Proposition 22 there exists a partitioned, $\approx$-compatible, $\sim$-saturated, and unitary fta $\mathcal{B}$ with labelling set $\operatorname{Rat}(H)$ such that

$$
\mathrm{L}(\mathcal{B}) \cap \operatorname{Red}(H, t)=\left[\mathrm{L}\left(\mathcal{A}_{3}\right)\right] \approx \cap \operatorname{Red}(H, t) .
$$

Let us summarize the connections between the rational set $K \subseteq G$ and the successive automata $\mathcal{A}, \mathcal{A}_{1}, \mathcal{A}_{2}, \mathcal{A}_{3}$, and $\mathcal{B}$ :

$$
\begin{array}{rlrl}
K & =\pi(\mathrm{L}(\mathcal{A})) & & \subseteq G \\
\mathrm{~L}\left(\mathcal{A}_{1}\right) & =\mathrm{L}(\mathcal{A}) \rightarrow \rightarrow_{\mathrm{ST}}^{*} & & \subseteq\left(X \cup\left\{t, t^{-1}\right\}\right)^{*} \\
\mathrm{~L}\left(\mathcal{A}_{2}\right) & =\pi_{t}\left(\mathrm{~L}\left(\mathcal{A}_{1}\right)\right) & & \subseteq H *\left\{t, t^{-1}\right\}^{*} \\
\mathrm{~L}\left(\mathcal{A}_{3}\right) & =\mathrm{L}\left(\mathcal{A}_{2}\right) & & \subseteq H *\left\{t, t^{-1}\right\}^{*} \\
\mathrm{~L}(\mathcal{B}) \cap \operatorname{Red}(H, t) & =\left[\mathrm{L}\left(\mathcal{A}_{3}\right)\right]_{\approx} \cap \operatorname{Red}(H, t) & \subseteq H *\left\{t, t^{-1}\right\}^{*}
\end{array}
$$

Since $\pi=\pi_{t} \circ \pi_{G}(9)$, equation (60) implies

$$
\pi_{G}^{-1}(K)=\pi_{G}^{-1}\left(\pi_{G}\left(\pi_{t}(\mathrm{~L}(\mathcal{A}))\right)\right)=\left[\pi_{t}(\mathrm{~L}(\mathcal{A}))\right]_{\approx} .
$$

By $(61),[\mathrm{L}(\mathcal{A})]_{\equiv}=\left[\mathrm{L}\left(\mathcal{A}_{1}\right)\right]_{\equiv}$, hence $\left[\pi_{t}(\mathrm{~L}(\mathcal{A}))\right]_{\approx}=\left[\pi_{t}\left(\mathrm{~L}\left(\mathcal{A}_{1}\right)\right)\right]_{\approx}$ and thus

$$
\pi_{G}^{-1}(K)=\left[\pi_{t}\left(\mathrm{~L}\left(\mathcal{A}_{1}\right)\right)\right] \approx .
$$

Using (62) and (63) we get

$$
\pi_{G}^{-1}(K)=\left[\mathrm{L}\left(\mathcal{A}_{2}\right)\right]_{\approx}=\left[\mathrm{L}\left(\mathcal{A}_{3}\right)\right]_{\approx} .
$$

Finally, by (64) we have

$$
\pi_{G}^{-1}(K) \cap \operatorname{Red}(H, t)=\mathrm{L}(\mathcal{B}) \cap \operatorname{Red}(H, t) .
$$

We have obtained point (3) of Proposition 33.

$(3) \Rightarrow(1)$ : Let $\mathcal{B}$ be some partitioned, $\approx$-compatible, and $\sim$-saturated fta with labelling set $\operatorname{Rat}(H)$ such that

$$
\pi_{G}^{-1}(K) \cap \operatorname{Red}(H, t)=\mathrm{L}(\mathcal{B}) \cap \operatorname{Red}(H, t) .
$$

Let $\mathcal{L}_{\mathcal{B}} \subseteq \operatorname{Rat}(H) \cup\left\{\{t\},\left\{t^{-1}\right\}\right\}$ be the set of labels of the transitions of the fta $\mathcal{B}$. We can substitute in $\mathcal{B}$ every $L$-labelled edge with $L \in \operatorname{Rat}(H)$ by a finite automaton over the generating set $X$ of $H$. In this way, we see that

$$
\mathrm{L}(\mathcal{B}) \in \operatorname{Rat}\left(H *\left\{t, t^{-1}\right\}^{*}\right) .
$$

As every $t$-sequence is equivalent (modulo $\approx$ ) with at least one reduced sequence, we have

$$
K=\pi_{G}\left(\pi_{G}^{-1}(K) \cap \operatorname{Red}(H, t)\right)
$$

With (65) we get

$$
K=\pi_{G}(\mathrm{~L}(\mathcal{B}) \cap \operatorname{Red}(H, t))
$$


Since $\mathcal{B}$ is $\approx$-compatible, we have

$$
\pi_{G}(\mathrm{~L}(\mathcal{B})) \subseteq \pi_{G}([\mathrm{~L}(\mathcal{B}) \cap \operatorname{Red}(H, t)] \approx)=\pi_{G}(\mathrm{~L}(\mathcal{B}) \cap \operatorname{Red}(H, t)) .
$$

Since also $\pi_{G}(\mathrm{~L}(\mathcal{B}) \cap \operatorname{Red}(H, t)) \subseteq \pi_{G}(\mathrm{~L}(\mathcal{B}))$, (68) implies

$$
K=\pi_{G}(\mathrm{~L}(\mathcal{B})) .
$$

As any homomorphic image of a rational subset is rational, $K$ is a rational subset of $G$.

\subsection{Transfer results for rational sets}

In this section we prove the following theorem, which allows to transfer important properties from $\operatorname{Rat}(H)$ to $\operatorname{Rat}(G)$.

Theorem 37. Let $G=\left\langle H, t ; t^{-1} a t=\varphi(a)(a \in A)\right\rangle$, where $H$ is a countably generated monoid with finite subgroups $A$ and $B$, and $\varphi: A \rightarrow B$ is an isomorphism. Then we have:

(1) If $\operatorname{Rat}(H)$ is closed under intersection, then so is $\operatorname{Rat}(G)$.

(2) If $\operatorname{Rat}(H)$ is closed under complement, then so is $\operatorname{Rat}(G)$.

(3) If the membership problem for rational subsets of $H$ is decidable, then so is it for $G$.

(4) If the emptiness problem for boolean combinations of rational subsets of $H$ is decidable, then so is it for $G$.

Proof. For statement (1), let $K_{1}, K_{2} \in \operatorname{Rat}(G)$ and $K=K_{1} \cap K_{2}$. By Proposition 33 there are partitioned, $\approx$-compatible, and $\sim$-saturated fta $\mathcal{A}_{1}$ and $\mathcal{A}_{2}$ with labelling set $\operatorname{Rat}(H)$ such that

$$
\pi_{G}^{-1}\left(K_{i}\right) \cap \operatorname{Red}(H, t)=\mathrm{L}\left(\mathcal{A}_{i}\right) \cap \operatorname{Red}(H, t)
$$

for $i \in\{1,2\}$. Hence,

$$
\begin{aligned}
\pi_{G}^{-1}(K) \cap \operatorname{Red}(H, t) & =\pi_{G}^{-1}\left(K_{1}\right) \cap \pi_{G}^{-1}\left(K_{2}\right) \cap \operatorname{Red}(H, t) \\
& =\mathrm{L}\left(\mathcal{A}_{1}\right) \cap \mathrm{L}\left(\mathcal{A}_{2}\right) \cap \operatorname{Red}(H, t) .
\end{aligned}
$$

Let us consider the partitioned fta

$$
\mathcal{A}=\mathcal{A}_{1} \times \mathcal{A}_{2} .
$$

By Lemma $15, \mathrm{~L}(\mathcal{A})=\mathrm{L}\left(\mathcal{A}_{1}\right) \cap \mathrm{L}\left(\mathcal{A}_{2}\right)$, and by Lemma $17, \mathcal{A}$ is $\approx$-compatible and $\sim$-saturated. Moreover, since $\operatorname{Rat}(H)$ is closed under intersection, the labelling set of $\mathcal{A}$ is still $\operatorname{Rat}(H)$. Hence $K$ fulfils point (3) of Proposition 33. By point (1) of Proposition $33, K$ is a rational subset of $G$.

For statement (2), let $K \in \operatorname{Rat}(G)$. By point (3) of Proposition 33 we have

$$
\pi_{G}^{-1}(K) \cap \operatorname{Red}(H, t)=\mathrm{L}(\mathcal{B}) \cap \operatorname{Red}(H, t)
$$


for a partitioned, $\approx$-compatible, and $\sim$-saturated fta $\mathcal{B}$ with labelling set $\operatorname{Rat}(H)$. By applying Proposition 28 to $\mathcal{B}$, we obtain

$$
\pi_{G}^{-1}(K) \cap \operatorname{Red}(H, t)=\mathrm{L}(\mathcal{C})
$$

for some partitioned, $\sim$-saturated, deterministic, and complete fta $\mathcal{C}$. The labelling set of this fta is $\operatorname{Bool}(\operatorname{Rat}(H))$, which equals $\operatorname{Rat}(H)$, since $\operatorname{Rat}(H)$ is supposed to be closed under complement.

Now take the complement automaton $\overline{\mathcal{C}}$ of $\mathcal{C}$, see Definition 19. By Lemma 20 it is a partitioned and $\sim$-saturated fta with

$$
\mathrm{L}(\overline{\mathcal{C}})=\operatorname{Red}(H, t) \backslash \mathrm{L}(\mathcal{C}) \subseteq \operatorname{Red}(H, t) .
$$

In particular, $\overline{\mathcal{C}}$ is also $\approx$-compatible. We obtain

$$
\begin{aligned}
\pi_{G}^{-1}(G \backslash K) \cap \operatorname{Red}(H, t) & =\left(H *\left\{t, t^{-1}\right\}^{*} \backslash \pi_{G}^{-1}(K)\right) \cap \operatorname{Red}(H, t) \\
& =\operatorname{Red}(H, t) \backslash\left(\pi_{G}^{-1}(K) \cap \operatorname{Red}(H, t)\right) \\
& =\operatorname{Red}(H, t) \backslash \mathrm{L}(\mathcal{C}) \\
& =\mathrm{L}(\overline{\mathcal{C}}) \\
& =\mathrm{L}(\overline{\mathcal{C}}) \cap \operatorname{Red}(H, t) .
\end{aligned}
$$

Hence, Proposition 33 implies that $G \backslash K$ is a rational subset of $G$. This proves statement (2).

For statement (3) suppose that the membership problem for rational subsets of $H$ is decidable. Let $K=\pi(\mathrm{L}(\mathcal{A}))$ for a given finite automaton $\mathcal{A}$ over the free monoid $\left(X \cup\left\{t, t^{-1}\right\}\right)^{*}$. By point (3) of Proposition 33 we have

$$
\pi_{G}^{-1}(K) \cap \operatorname{Red}(H, t)=\mathrm{L}(\mathcal{B}) \cap \operatorname{Red}(H, t)
$$

for a partitioned, $\approx$-compatible, and $\sim$-saturated fta $\mathcal{B}$ with labelling set $\operatorname{Rat}(H)$. Moreover, since the membership problem for languages in $\operatorname{Rat}(H)$ is decidable, $\mathcal{B}$ can be constructed effectively from $\mathcal{A}$.

Now, to a given word $u \in\left(X \cup\left\{t, t^{-1}\right\}\right)^{*}$ we first apply (effectively) as long as possible the rules of the semi-Thue system ST in order to obtain a word $v \in$ $\left(X \cup\left\{t, t^{-1}\right\}\right)^{*}$ of the form

$$
v=v_{0} t^{\alpha_{1}} v_{1} \cdots t^{\alpha_{i}} v_{i} \cdots t^{\alpha_{p}} v_{p}
$$

where $p \geq 0, \alpha_{i} \in\{1,-1\}, v_{i} \in X^{*}$, and $\pi_{t}(v) \in \operatorname{Red}(H, t)$. Note that ST contains infinitely many rules, but nevertheless we can compute effectively such a word $v$ : since the word problem is decidable for $H$, we can decide whether $[w]_{\equiv_{H}} \in A$ (resp. $[w]_{\equiv_{H}} \in B$ for a given word $w \in X^{*}$; this allows us to apply rules from ST in an effective way.

Since the membership problem is decidable for the labelling sets of $\mathcal{B}$, we can compute all the binary relations $\mu_{\mathcal{B}}\left(\pi_{t}\left(v_{i}\right)\right), \mu_{\mathcal{B}}(t)$, and $\mu_{\mathcal{B}}\left(t^{-1}\right)$. This allows us to compute the binary relation $\mu_{\mathcal{B}}\left(\pi_{t}(v)\right)$. Since $u \equiv v$, we have $\pi(u) \in K$ if and only if $\pi(v) \in K$ if and only if $\pi_{t}(v) \in \pi_{G}^{-1}(K)$ if and only if $\pi_{t}(v) \in \mathrm{L}(\mathcal{B})$ if and only if $\mu_{\mathcal{B}}\left(\pi_{t}(v)\right) \cap \mathbf{I}_{\mathcal{B}} \times \mathrm{T}_{\mathcal{B}} \neq \emptyset$. 
Let us finally prove statement (4). Assume that the emptiness problem for boolean combinations of rational subsets of $H$ is decidable. Let $K_{1}, \ldots, K_{p}$ be rational subsets of $G$ defined as $K_{i}=\pi\left(\mathrm{L}\left(\mathcal{A}_{i}\right)\right)$ for some given finite automaton $\mathcal{A}_{i}$ over the free monoid $\left(X \cup\left\{t, t^{-1}\right\}\right)^{*}$. Following the construction described for statement (2) of Theorem 37, we can construct effectively for every $i \in\{1, \ldots, p\}$ a partitioned, $\sim$-saturated, deterministic, and complete fta $\mathcal{C}_{i}$, whose labelling set is $\operatorname{Bool}(\operatorname{Rat}(H))$, and such that

$$
\mathrm{L}\left(\mathcal{C}_{i}\right)=\pi_{G}^{-1}\left(K_{i}\right) \cap \operatorname{Red}(H, t) .
$$

The boolean operations $\cap$ and complement over $\sim$-saturated subsets of $\operatorname{Red}(H, t)$ can be translated into the product and complement operation, respectively, over partitioned, -saturated, deterministic, and complete fta reading only reduced sequences (for products, we have to use Lemma 17 and 18, for complements we use Lemma 20). Hence, from a given boolean combination $K$ of the sets $K_{i}$, one can compute effectively a corresponding fta $\mathcal{C}$, whose labelling set is $\operatorname{Bool}(\operatorname{Rat}(H))$, and such that

$$
\mathrm{L}(\mathcal{C})=\pi_{G}^{-1}(K) \cap \operatorname{Red}(H, t) .
$$

Emptiness of the set $K$ reduces to emptiness of $\mathrm{L}(\mathcal{C})$, which itself reduces to the emptiness problem for the label sets of $\mathcal{C}$. Hence, under the hypothesis that emptiness is decidable for languages in $\operatorname{Bool}(\operatorname{Rat}(H))$, we can test whether $\mathrm{L}(\mathcal{C})=\emptyset$ or not.

Remark 38. For a given monoid $\mathbb{M}$, closure of Rat $(\mathbb{M})$ under complement implies closure of Rat $(\mathbb{M})$ under intersection, but the converse does not hold in general: we give examples of such monoids $\mathbb{M}$ where $\operatorname{Rat}(\mathbb{M})$ is closed under intersection but not under complement in Appendix B. This shows that points (1) and (2) of Theorem 37 cannot be deduced in a straightforward manner one from the other.

The same is also true for (3) and (4): For the group $F \times \mathbb{Z}$ (where $F$ is the free group of rank 2), the membership problem for rational subsets is decidable [KSS06], whereas the inclusion problem for rational subsets (which is a special instance of the emptiness problem for boolean combinations of rational subsets) is undecidable [AH89].

\subsection{Transfer results for finitely generated subgroups}

We suppose in this section that $H=X^{*} / \equiv$ (and hence $G$ ) is a group. We assume moreover that the generating set $X$ is endowed with an involution $x \mapsto x^{-1}$ such that $x x^{-1} \equiv x^{-1} x \equiv \varepsilon$. The following subset of $\mathcal{P}(H)$ will serve for labelling fta in the sequel:

$$
\mathcal{S}=\{K h \mid K \text { is a finitely generated subgroup of } H, h \in H\}
$$

Note that every singleton subset $\{h\}$ belongs to $\mathcal{S}$. 


\subsubsection{Normalization of automata}

Let us prove first a normalization lemma for finitely generated subgroups of $G$, which parallels Proposition 33:

Proposition 39. Let $K$ be a subgroup of $G$. The following are equivalent:

(1) $K$ is finitely generated.

(2) $K$ is rational.

(3) There exists some finite automaton $\mathcal{A}$ over the free monoid $\left(X \cup\left\{t, t^{-1}\right\}\right)^{*}$ such that $K=\pi(\mathrm{L}(\mathcal{A}))$.

(4) $\pi_{G}^{-1}(K) \cap \operatorname{Red}(H, t)=\mathrm{L}(\mathcal{B}) \cap \operatorname{Red}(H, t)$ for some partitioned, $\approx$-compatible, and $\sim$-saturated fta $\mathcal{B}$ with labelling set $\mathcal{S}$.

Moreover, in (4), if the generalized word problem of $H$ is decidable, then $\mathcal{B}$ can be constructed effectively from $\mathcal{A}$ in (3).

Proof. We follow the arguments from the proof of Proposition 33.

$(1) \Leftrightarrow(2)$ : This is just Theorem 1 .

$(2) \Leftrightarrow(3)$ : This has been shown in Proposition 33 .

$(3) \Rightarrow(4)$ : We must here slightly adapt the corresponding proof given for Proposition 33. Let us start with a finite automaton $\mathcal{A}=\langle\mathcal{X}, \mathrm{Q}, \delta, \mathrm{I}, \mathrm{T}\rangle$ over the free monoid $\left(X \cup\left\{t, t^{-1}\right\}\right)^{*}$ such that

$$
K=\pi(\mathrm{L}(\mathcal{A}))
$$

Claim 40. There exists a finite automaton $\mathcal{A}_{1}$ over the free monoid $\left(X \cup\left\{t, t^{-1}\right\}\right)^{*}$ such that

$$
\mathrm{L}(\mathcal{A}) \subseteq \mathrm{L}\left(\mathcal{A}_{1}\right)=\mathrm{L}\left(\mathcal{A}_{1}\right) \rightarrow_{\mathrm{ST}}^{*} \subseteq[\mathrm{L}(\mathcal{A})]_{\equiv}
$$

Moreover, if the generalized word problem of $H$ is decidable, then $\mathcal{A}_{1}$ can be constructed effectively from $\mathcal{A}$.

We assume that $\mathcal{A}$ is trim, i.e., every state of $\mathcal{A}$ is accessible from an initial state and co-accessible from a final state. We start with

$$
\delta_{1}=\delta \cup\left\{\left(r, c^{-1}, q\right) \mid c \in X \cup\left\{t, t^{-1}\right\},(q, c, r) \in \delta\right\} .
$$

Since $\pi(\mathrm{L}(\mathcal{A}))$ is a group, we have

$$
\mathrm{L}(\mathcal{A}) \subseteq \mathrm{L}\left(\left\langle\mathcal{X}, \mathrm{Q}, \delta_{1}, \mathrm{I}, \mathrm{T}\right\rangle\right) \subseteq[\mathrm{L}(\mathcal{A})]_{\equiv} .
$$

For all states $q, q^{\prime} \in \mathrm{Q}$ we define $\ell\left(\delta_{1}, q, q^{\prime}\right)$ as a shortest word over $X$ which labels a $\delta_{1}$-path from $q$ to $q^{\prime}$. We saturate $\delta_{1}$ under the following inference rules, where 
$u \in X^{*}:$

$$
\begin{gathered}
\frac{p \stackrel{t^{-1}}{\longrightarrow} \delta_{1} q \stackrel{u}{\rightarrow} \delta_{1} q \stackrel{\ell\left(\delta_{1}, q, q^{\prime}\right)}{\longrightarrow} \delta_{1} q^{\prime} \stackrel{t}{\rightarrow} \delta_{1} r \wedge u \ell\left(\delta_{1}, q, q^{\prime}\right) \equiv_{H} a \in A}{p \stackrel{\varphi(a)}{\longrightarrow} \delta_{1} r} \\
\frac{p \stackrel{t}{\rightarrow} \delta_{1} q \stackrel{u}{\rightarrow} \delta_{1} q \stackrel{\ell\left(\delta_{1}, q, q^{\prime}\right)}{\longrightarrow} \delta_{1} q^{\prime} \stackrel{t^{-1}}{\longrightarrow} \delta_{1} r \wedge u \ell\left(\delta_{1}, q, q^{\prime}\right) \equiv_{H} b \in B}{p \stackrel{\varphi^{-1}(b)}{\longrightarrow} \delta_{1} r}
\end{gathered}
$$

Let $\delta_{1}$ be the resulting transition relation and let

$$
\mathcal{A}_{1}=\left\langle\mathcal{X} \cup A \cup B, \mathrm{Q}, \delta_{1}, \mathrm{I}, \mathrm{T}\right\rangle .
$$

It satisfies (70). For this notice that if $q \stackrel{v}{\rightarrow} \delta_{1} q^{\prime}$ for a word $v \in X^{*}$ with $v \equiv_{H}$ $a \in A$ then $v \ell\left(\delta_{1}, q, q^{\prime}\right)^{-1} \ell\left(\delta_{1}, p, q\right) \equiv_{H} a$ and $q \stackrel{v \ell\left(\delta_{1}, q, q^{\prime}\right)^{-1}}{\longrightarrow} \delta_{1} q$, i.e., the word $v \ell\left(\delta_{1}, q, q^{\prime}\right)^{-1}$ is a candidate for $u$ in the above rule (71).

It remains to show the effectiveness statement in Claim 40. Let us consider for every value of $\delta_{1}$ and every $q \in \mathrm{Q}$ the set

$$
P_{1}\left(\delta_{1}, q\right)=\left\{u \in X^{*} \mid q \stackrel{u}{\rightarrow} \delta_{1} q\right\} .
$$

By Theorem $1, \pi_{t}\left(P_{1}\left(\delta_{1}, q\right)\right)$ is a finitely generated subgroup of $H$, and by the proof given in Section 2.2, a finite set of generators for it can be explicitly computed. If the generalized word problem of $H$ is decidable, then the validity of the hypothesis of the inference rules (71) and (72) can be algorithmically tested so that $\delta_{1}$ can be algorithmically computed. This proves Claim 40 .

Claim 41. There exists $a \approx$-compatible fta $\mathcal{A}_{2}$ over $H *\left\{t, t^{-1}\right\}^{*}$ with labelling set $\mathcal{S}$ such that

$$
\mathrm{L}\left(\mathcal{A}_{2}\right)=\pi_{t}\left(\mathrm{~L}\left(\mathcal{A}_{1}\right)\right) .
$$

Moreover, $\mathcal{A}_{2}$ can be constructed effectively from $\mathcal{A}_{1}$.

Analogously to the proof of Claim 35, we do a collecting process:

$$
\begin{aligned}
\delta_{2}= & \left\{\left(q, t^{\alpha}, r\right) \mid \alpha \in\{1,-1\},\left(q, t^{\alpha}, r\right) \in \delta_{1}\right\} \cup \\
& \left\{\left(q, \pi_{t}\left(P_{1}\left(\delta_{1}, q\right) \ell\left(\delta_{1}, q, r\right)\right), r\right) \mid q, r \in \mathrm{Q}\right\} .
\end{aligned}
$$

This step can be shown to be effective by the arguments already used for the proof of Claim 40. Clearly (73) holds. Moreover, (70) implies that $\mathrm{L}\left(\mathcal{A}_{1}\right) \subseteq\left[\mathrm{L}\left(\mathcal{A}_{1}\right) \cap\right.$ $\operatorname{Irr}(\mathrm{ST})]_{\equiv}$. Now $\mathrm{L}\left(\mathcal{A}_{2}\right) \subseteq\left[\mathrm{L}\left(\mathcal{A}_{2}\right) \cap \operatorname{Red}(H, t)\right]_{\approx}$ can be shown in the same way as in the proof of Claim 35 . Hence, $\mathcal{A}_{2}$ is $\approx$-compatible.

Claim 42. There exists a partitioned and $\approx$-compatible fta $\mathcal{A}_{3}$ over $H *\left\{t, t^{-1}\right\}^{*}$ with labelling set $\mathcal{S}$ and such that

$$
\mathrm{L}\left(\mathcal{A}_{3}\right)=\mathrm{L}\left(\mathcal{A}_{2}\right) .
$$

Moreover, $\mathcal{A}_{3}$ can be constructed effectively from $\mathcal{A}_{2}$. 
As for Claim 36 set $\mathcal{A}_{3}=\mathcal{A}_{2} \times \mathcal{G}_{6}$.

Let us now apply Proposition 22 to the set $\mathcal{F}=\mathcal{S}$ and the fta $\mathcal{A}_{3}$, and let $\mathcal{B}$ be the resulting partitioned, $\approx$-compatible, and $\sim$-saturated fta. Note that the labelling set of $\mathcal{B}$ is still $\mathcal{S}$ : If $K h$ labels a transition of $\mathcal{A}_{3}$, where $K$ is a finitely generated subgroup of $H$ and $h \in H$, then a transition of $\mathcal{B}$ may be labelled with $c(K h) c^{\prime}$, where $c, c^{\prime} \in A \cup B$. But we have $c(K h) c^{\prime}=\left(c K c^{-1}\right)\left(c h c^{\prime}\right) \in \mathcal{S}$.

We obtain the following relationships between the automata $\mathcal{A}, \mathcal{A}_{1}, \mathcal{A}_{2}, \mathcal{A}_{3}$, and $\mathcal{B}:$

$$
\begin{array}{rlrl}
K & =\pi(\mathrm{L}(\mathcal{A})) & & \subseteq G \\
\mathrm{~L}(\mathcal{A}) & \subseteq \mathrm{L}\left(\mathcal{A}_{1}\right) \subseteq[\mathrm{L}(\mathcal{A})]_{\equiv} & & \subseteq\left(X \cup\left\{t, t^{-1}\right\}\right)^{*} \\
\mathrm{~L}\left(\mathcal{A}_{2}\right) & =\pi_{t}\left(\mathrm{~L}\left(\mathcal{A}_{1}\right)\right) & & \subseteq H *\left\{t, t^{-1}\right\}^{*} \\
\mathrm{~L}\left(\mathcal{A}_{3}\right) & =\mathrm{L}\left(\mathcal{A}_{2}\right) & & \subseteq H *\left\{t, t^{-1}\right\}^{*} \\
\mathrm{~L}(\mathcal{B}) \cap \operatorname{Red}(H, t) & =\left[\mathrm{L}\left(\mathcal{A}_{3}\right)\right]_{\approx} \cap \operatorname{Red}(H, t) & \subseteq H *\left\{t, t^{-1}\right\}^{*}
\end{array}
$$

From $(76)$ we obtain $[\mathrm{L}(\mathcal{A})]_{\equiv}=\left[\mathrm{L}\left(\mathcal{A}_{1}\right)\right]_{\equiv}$. Now, we can deduce $\pi_{G}^{-1}(K) \cap \operatorname{Red}(H, t)=$ $\mathrm{L}(\mathcal{B}) \cap \operatorname{Red}(H, t)$ in the same way as in the corresponding part of the proof of Proposition 33.

$(4) \Rightarrow(2)$ : This follows from Proposition 33 since, $\mathcal{S} \subseteq \operatorname{Rat}(H)$.

Using Proposition 39, we can next prove a transfer theorem for finitely generated subgroups:

Theorem 43. Let $G=\left\langle H, t ; t^{-1} a t=\varphi(a)(a \in A)\right\rangle$, where $H$ is a countably generated group with finite subgroups $A$ and $B$, and $\varphi: A \rightarrow B$ is an isomorphism. Then we have:

(1) If $H$ has a decidable generalized word problem, then so has $G$.

(2) If $H$ has the Howson property, then so has $G$.

(3) If $H$ has the LERF property, then so has $G$.

Proof. For property (1), let $u_{1}, \ldots, u_{n}, u \in\left(X \cup\left\{t, t^{-1}\right\}\right)^{*}$. Let $K \subseteq G$ be the subgroup generated by $\left\{\pi\left(u_{1}\right), \ldots, \pi\left(u_{n}\right)\right\}$. We give an algorithm for deciding whether $\pi(u) \in K$ or not:

Step 1: We construct from $u_{1}, \ldots, u_{n}$ a finite automaton $\mathcal{A}$ over the free monoid $\left(X \cup\left\{t, t^{-1}\right\}\right)^{*}$ such that

$$
K=\pi(\mathrm{L}(\mathcal{A})) .
$$

Step 2: Using Proposition 39 we can compute from $\mathcal{A}$ effectively a partitioned, $\approx$-compatible, and $\sim$-saturated fta $\mathcal{B}$ with labelling set $\mathcal{S}$ such that

$$
\pi_{G}^{-1}(K) \cap \operatorname{Red}(H, t)=\mathrm{L}(\mathcal{B}) \cap \operatorname{Red}(H, t) .
$$


Step 3: Starting from the word $u \in\left(X \cup\left\{t, t^{-1}\right\}\right)^{*}$ and applying (effectively) the rules of ST as long as possible, one computes a word $v \in\left(X \cup\left\{t, t^{-1}\right\}\right)^{*}$ of the form

$$
v=v_{0} t^{\alpha_{1}} v_{1} \cdots t^{\alpha_{i}} v_{i} \cdots t^{\alpha_{p}} v_{p}
$$

where $p \geq 0, \alpha_{i} \in\{1,-1\}, v_{i} \in X^{*}$, and $\pi_{t}(v) \in \operatorname{Red}(H, t)$.

Step 4: From $\mathcal{B}$ one can compute all the binary relations $\mu_{\mathcal{B}}\left(\pi_{t}\left(v_{i}\right)\right), \mu_{\mathcal{B}}(t)$, and $\mu_{\mathcal{B}}\left(t^{-1}\right)$. This allows to compute the binary relation $\mu_{\mathcal{B}}\left(\pi_{t}(v)\right)$. Since $u \equiv v$, we have $\pi(u) \in K$ if and only if $\pi(v) \in K$ if and only if $\pi_{t}(v) \in \pi_{G}^{-1}(K)$ if and only if $\pi_{t}(v) \in \mathrm{L}(\mathcal{B})$ if and only if $\mu_{\mathcal{B}}\left(\pi_{t}(v)\right) \cap \mathbf{I}_{\mathcal{B}} \times \mathrm{T}_{\mathcal{B}} \neq \emptyset$.

Let us now prove (2): Suppose that $H$ has the Howson property. Let $K_{1}$ and $K_{2}$ be two finitely generated subgroups of $G$. By Proposition 39, there exist partitioned, $\approx$-compatible, and $\sim$-saturated fta $\mathcal{B}_{1}$ and $\mathcal{B}_{2}$ with labelling set $\mathcal{S}$ such that

$$
\pi_{G}^{-1}\left(K_{i}\right) \cap \operatorname{Red}(H, t)=\mathrm{L}\left(\mathcal{B}_{i}\right) \cap \operatorname{Red}(H, t)
$$

for $i \in\{1,2\}$. Let

$$
\mathcal{B}=\mathcal{B}_{1} \times \mathcal{B}_{2} .
$$

By the same arguments as for point $(1)$ of Theorem $37, \mathcal{B}$ is partitioned, $\approx-$ compatible, and $\sim$-saturated. Let us check that its transition labels belong to $\mathcal{S}$ : Every label $L$ of $\mathcal{B}$ either belongs to $\left\{\{t\},\left\{t^{-1}\right\}\right\}$ or has the form

$$
L=F_{1} h_{1} \cap F_{2} h_{2} \neq \emptyset
$$

for finitely generated subgroups $F_{1}, F_{2} \leq H$ and $h_{1}, h_{2} \in H$. Since $F_{1} h_{1} \cap F_{2} h_{2} \neq \emptyset$, there exist $f_{1} \in F_{1}$ and $f_{2} \in F_{2}$ such that $f_{1} h_{1}=f_{2} h_{2}$ in $H$. We thus have

$$
\begin{aligned}
F_{1} h_{1} \cap F_{2} h_{2} & =\left(\left(F_{1} f_{1}^{-1} f_{2}\right) \cap F_{2}\right) h_{2} \\
& =\left(F_{1} f_{2} \cap F_{2}\right) h_{2} \\
& =\left(F_{1} \cap F_{2}\right) f_{2} h_{2} .
\end{aligned}
$$

By the Howson property for $H, F_{1} \cap F_{2}$ is a finitely generated subgroup of $H$. Hence, $\left(F_{1} \cap F_{2}\right) f_{2} h_{2}$ belongs to $\mathcal{S}$. Thus

$$
\pi_{G}^{-1}\left(K_{1} \cap K_{2}\right) \cap \operatorname{Red}(H, t)=\mathrm{L}(\mathcal{B}) \cap \operatorname{Red}(H, t)
$$

for a partitioned, $\approx$-compatible, and $\sim$-saturated fta $\mathcal{B}$ with labelling set $\mathcal{S}$. By Proposition 39 this implies that $K_{1} \cap K_{2}$ is finitely generated.

Finally, we prove point (3). In order to treat the LERF property (see Definition 3 ) we have to introduce a technical notion about finite $t$-automata, which is stronger than $\approx$-compatibility:

Definition 44. Let $\mathcal{B}=\langle\mathcal{L}, \mathrm{Q}, \tau, \delta, \mathrm{I}, \mathrm{T}\rangle$ be a partitioned fta. It is said to be downwards-closed (briefly d-closed) if for all $q_{1}, q_{2}, q_{3}, q_{4}, q_{5}, q_{6} \in \mathrm{Q}, h_{2}, h_{6} \in H$, $a \in A$, and $b \in B$ such that $\tau\left(q_{1}\right) \neq \tau\left(q_{2}\right)$ and $\tau\left(q_{5}\right) \neq \tau\left(q_{6}\right)$ :

$$
\begin{aligned}
& q_{1} \stackrel{h_{2}}{\longrightarrow} q_{2} \stackrel{t^{-1}}{\longrightarrow} q_{3} \stackrel{a}{\rightarrow}_{\mathcal{B}} q_{4} \stackrel{t}{\rightarrow} \mathcal{B} q_{5}{\stackrel{h_{6}}{\longrightarrow}}_{\mathcal{B}} q_{6} \Rightarrow q_{1} \stackrel{h_{2} \varphi(a) h_{6}}{\longrightarrow} q_{6} \\
& q_{1} \stackrel{h_{2}}{\longrightarrow} q_{2} q_{2} \stackrel{t}{\rightarrow} q_{3} \stackrel{b}{\longrightarrow}_{\mathcal{B}} q_{4} \stackrel{t^{-1}}{\longrightarrow} \mathcal{B} q_{5} \stackrel{h_{6}}{\longrightarrow} q_{6} \Rightarrow q_{1} \stackrel{h_{2} \varphi^{-1}(b) h_{6}}{\longrightarrow} q_{6} q_{6}
\end{aligned}
$$


Lemma 45. A partitioned and d-closed fta is $\approx$-compatible.

Proof. Assume that the partitioned fta $\mathcal{B}=\langle\mathcal{L}, \mathrm{Q}, \tau, \delta, \mathrm{I}, \mathrm{T}\rangle$ is $d$-closed and let $s \in \mathrm{L}(\mathcal{B})$. We have to show that $s \in[\mathrm{L}(\mathcal{B}) \cap \operatorname{Red}(H, t)] \approx$. For this, assume that $s$ is not reduced, i.e., $s=s_{1} t^{-1} a t s_{2}$. Since $s \in \mathrm{L}(\mathcal{B})$, there exists a path in $\mathcal{B}$, labelled with $s$, leading from a state of type $(1, H)$ to a state of type $(1,1)$. Since $\mathcal{B}$ is partitioned, the sequences $s_{1}$ and $s_{2}$ cannot be empty: note that no $t$-labelled (resp. $t^{-1}$-labelled) transitions exits (resp. enters) a state of type $(1, H)($ resp. $(1,1))$ in a partitioned fta. Hence we have $s=s_{1}^{\prime} h_{2} t^{-1} a t h_{6} s_{2}^{\prime}$ for some $h_{2}, h_{6} \in H$. Moreover, there exist states $q_{1}, q_{2}, q_{3}, q_{4}, q_{5}, q_{6} \in \mathrm{Q}$ such that (80) holds. Since $\mathcal{B}$ is partitioned and the path labelled with $s$ leads from a state of type $(1, H)$ to a state of type $(1,1)$, we have $\tau\left(q_{1}\right) \neq \tau\left(q_{2}\right)$ and $\tau\left(q_{5}\right) \neq \tau\left(q_{6}\right)$ (see the last statement in Lemma 7$)$. Hence, since $\mathcal{B}$ is $d$-closed, we have $q_{1} \stackrel{h_{2} \varphi(a) h_{6}}{\longrightarrow} \mathcal{B} q_{6}$, i.e., $s_{1}^{\prime}\left(h_{2} \varphi(a) h_{6}\right) s_{2}^{\prime} \in \mathrm{L}(\mathcal{B})$. By continuing in this way, we finally arrive at a reduced sequence $s^{\prime} \in \mathrm{L}(\mathcal{B}) \cap \operatorname{Red}(H, t)$ such that $s \approx s^{\prime}$.

Let us now continue with the proof for point (3) in Theorem 43. Assume that $H$ has the LERF property. We will use the alternative definition from Remark 4 . Let $g \in G$, let $K \leq G$ be some finitely generated subgroup of $G$, and let us suppose that

$$
g \notin K .
$$

By Proposition 39 , there exists a partitioned, $\approx$-compatible, and $\sim$-saturated fta

$$
\mathcal{B}=\left\langle\mathcal{L}_{\mathcal{B}}, Q_{\mathcal{B}}, \tau_{\mathcal{B}}, \delta_{\mathcal{B}}, I_{\mathcal{B}}, T_{\mathcal{B}}\right\rangle
$$

with labelling set $\mathcal{S}$ such that

$$
\pi_{G}^{-1}(K) \cap \operatorname{Red}(H, t)=\mathrm{L}(\mathcal{B}) \cap \operatorname{Red}(H, t) .
$$

Claim 46. The fta $\mathcal{B}$ can be chosen to be $d$-closed.

Let us look at the fta $\mathcal{A}_{2}$ produced in the proof of Claim 41 by equation (74). This automaton is not partitioned but has both properties (80) and (81). The partitioned automaton $\mathcal{A}_{3}=\mathcal{A}_{2} \times \mathcal{G}_{6}$ still satisfies (80) and (81), since for all $q_{1}, \ldots, q_{6}$ fulfilling the hypothesis of (80) and (81), respectively, the edge $\left(\tau_{\mathcal{A}_{2}}\left(q_{1}\right), H, \tau_{\mathcal{A}_{2}}\left(q_{6}\right)\right)$ belongs to $\mathcal{E}_{6}$. Let us look finally at the fta $\mathcal{B}$ obtained from $\mathcal{A}_{3}$ by the construction in the proof of Proposition 22. Suppose that the hypothesis of implication (80) is fulfilled, i.e.,

$$
q_{1} g_{1}{\stackrel{h_{2}}{\longrightarrow}}_{\mathcal{B}} q_{2} g_{2} \stackrel{t^{-1}}{\longrightarrow} q_{3} g_{3} \stackrel{a}{\rightarrow}_{\mathcal{B}} q_{4} g_{4} \stackrel{t}{\rightarrow}_{\mathcal{B}} q_{5} g_{5} \stackrel{h_{6}}{\longrightarrow} \mathcal{B} q_{6} g_{6}
$$

where $q_{i} \in Q_{\mathcal{A}_{3}}, g_{i} \in \gamma\left(q_{i}\right), \tau_{\mathcal{B}}\left(q_{1} g_{1}\right) \neq \tau_{\mathcal{B}}\left(q_{2} g_{2}\right), \tau_{\mathcal{B}}\left(q_{5} g_{5}\right) \neq \tau_{\mathcal{B}}\left(q_{6} g_{6}\right)$, and hence $\tau_{\mathcal{A}_{3}}\left(q_{1}\right) \neq \tau_{\mathcal{A}_{3}}\left(q_{2}\right), \tau_{\mathcal{A}_{3}}\left(q_{5}\right) \neq \tau_{\mathcal{A}_{3}}\left(q_{6}\right)$. Assembling five of the diagrams described in Figure 4, we obtain from (84) in the fta $\mathcal{A}_{3}$ the path

$$
q_{1} \stackrel{g_{1} h_{2} g_{2}^{-1}}{\longrightarrow} \mathcal{A}_{3} q_{2} \stackrel{t^{-1}}{\longrightarrow} \mathcal{A}_{3} q_{3} \stackrel{g_{3} a g_{4}^{-1}}{\longrightarrow} \mathcal{A}_{3} q_{4} \stackrel{t}{\rightarrow} \mathcal{A}_{3} q_{5} \stackrel{g_{5} h_{6} g_{6}^{-1}}{\longrightarrow} \mathcal{A}_{3} q_{6},
$$




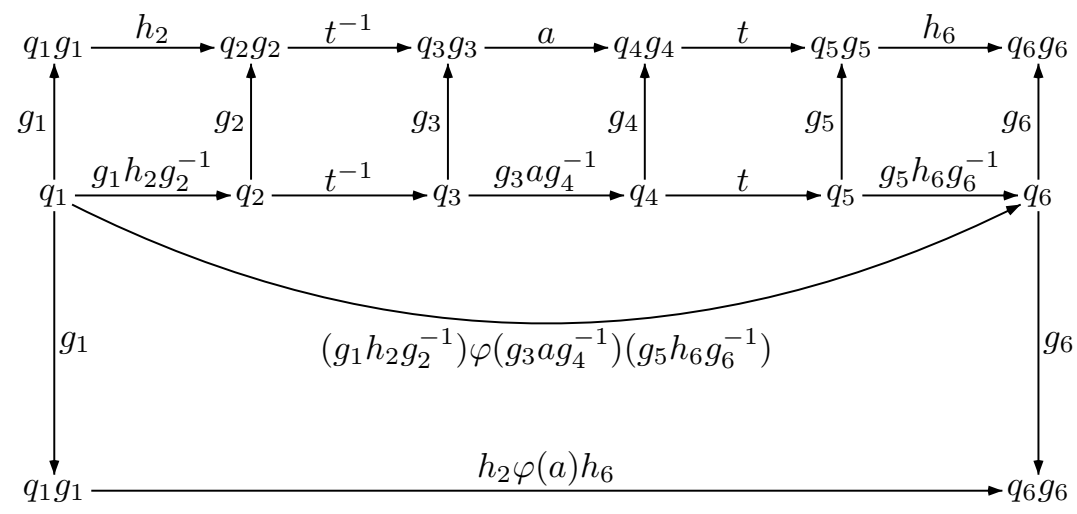

Fig. $5 . \mathcal{B}$ is $d$-closed

where

$$
\varphi^{-1}\left(g_{2}\right)=g_{3} \quad \text { and } \quad \varphi\left(g_{4}\right)=g_{5} .
$$

Since $\mathcal{A}_{3}$ is $d$-closed, we get

$$
q_{1} \stackrel{\left(g_{1} h_{2} g_{2}^{-1}\right) \varphi\left(g_{3} a g_{4}^{-1}\right)\left(g_{5} h_{6} g_{6}^{-1}\right)}{\longrightarrow} \mathcal{A}_{3} q_{6},
$$

i.e., with (86),

$$
q_{1} \stackrel{\left(g_{1} h_{2}\right) \varphi(a)\left(h_{6} g_{6}^{-1}\right)}{\longrightarrow} \mathcal{A}_{3} q_{6} .
$$

Using the left diagram in Figure 4, we obtain

$$
q_{1} g_{1} \stackrel{h_{2} \varphi(a) h_{6}}{\longrightarrow}{ }_{\mathcal{B}} q_{6} g_{6} .
$$

Thus, implication (80) holds, see also Figure 5. A similar verification can be done for implication (81). Claim 46 is thus established.

Let

$$
\mathcal{L}_{\mathcal{B}} \cap \mathcal{P}(H)=\left\{F_{0} r_{0}, \ldots, F_{\ell} r_{\ell}\right\}
$$

be the set of $H$-labels of $\mathcal{B}$, where $F_{i} \leq H$ is finitely generated and $r_{i} \in H$. Let

$$
s=h_{0} t^{\alpha_{1}} h_{1} \cdots t^{\alpha_{n}} h_{n} \in \operatorname{Red}(H, t)
$$

be a reduced sequence such that

$$
\pi_{G}(s)=g \notin K .
$$

By (83), (87), and the fact that $s$ is reduced, we have

$$
s \notin \mathrm{L}(\mathcal{B}) .
$$


For every subgroup $N^{\prime} \leq H$ let us consider the following properties:

$$
\begin{aligned}
& N^{\prime} \cap A=1=N^{\prime} \cap B \\
& \forall i \in\{0, \ldots, \ell\} \forall c \in A \cup B: c \in N^{\prime} F_{i} r_{i} \Leftrightarrow c \in F_{i} r_{i} \\
& \forall i \in\{0, \ldots, \ell\}, j \in\{0, \ldots, n\}: h_{j} \in N^{\prime} F_{i} r_{i} \Leftrightarrow h_{j} \in F_{i} r_{i} \\
& \forall j \in\{0, \ldots, n\}: h_{j} \in N^{\prime} A \Leftrightarrow h_{j} \in A \wedge h_{j} \in N^{\prime} B \Leftrightarrow h_{j} \in B
\end{aligned}
$$

Notice that the directions $\Leftarrow$ in $(90)-(92)$ is trivially satisfied for every subgroup $N^{\prime}$. As $H$ meets the LERF property, for each value of $i \in\{0, \ldots, \ell\}, j \in\{0, \ldots, n\}$, and each property $P$ among the above properties (89)-(92), there exists some normal subgroup of finite index $N(i, j, P) \unlhd H$ fulfilling the given property. For instance, for (90) one can argue as follows: if $c \notin F_{i} r_{i}$, then $c r_{i}^{-1} \notin F_{i}$. Since $F_{i}$ is finitely generated, the LERF property for $H$ implies the existence of a normal subgroup $N^{\prime} \leq H$ of finite index in $H$ such that $c r_{i}^{-1} \notin N F_{i}$, i.e., $c \notin N F_{i} r_{i}$.

Let us define

$$
N=\bigcap_{i, j, P} N(i, j, P) .
$$

This subgroup $N$ is normal and has finite index in $H$. Moreover, since every property among (89)-(92) is hereditary in the sense that if $N_{1} \leq N_{2} \leq H$ and $N_{2}$ meets the property, then $N_{1}$ meets the same property as well, $N$ fulfils simultaneously all the properties (89)-(92). Let us set

$$
G^{\prime}=\left\langle H, t ; x=1(x \in N), t^{-1} a t=\varphi(a)(a \in A)\right\rangle .
$$

Let $\pi^{\prime}: H \rightarrow H^{\prime}=H / N$ be the canonical projection. Due to (89), this morphism $\pi^{\prime}$ fulfils condition (19) from Section 3.1. Thus, the group $G^{\prime}$ is also the HNNextension of $H^{\prime}=H / N$ with stable letter $t$, associated subgroups $A, B$, and the partial isomorphism $\varphi$ :

$$
G^{\prime}=\left\langle H / N, t ; t^{-1} a t=\varphi(a)(a \in A)\right\rangle .
$$

Let us use the notation from Section 3.1. Let $\pi_{*}^{\prime}(\mathcal{B})$ be the $\sim^{\prime}$-saturated fta from Lemma 21, which satisfies

$$
\mathrm{L}\left(\pi_{*}^{\prime}(\mathcal{B})\right)=\pi_{*}^{\prime}(\mathrm{L}(\mathcal{B}))
$$

Let us define:

$$
s^{\prime}=\pi_{*}^{\prime}(s)=\pi^{\prime}\left(h_{0}\right) t^{\alpha_{1}} \pi^{\prime}\left(h_{1}\right) \cdots t^{\alpha_{n}} \pi^{\prime}\left(h_{n}\right) .
$$

Since $s$ is a reduced sequence in $H *\left\{t, t^{-1}\right\}^{*}$, condition (92) implies

$$
s^{\prime} \in \operatorname{Red}\left(H^{\prime}, t\right) \text {. }
$$

By (88) and (91) we have

$$
s^{\prime} \notin \mathrm{L}\left(\pi_{*}^{\prime}(\mathcal{B})\right) .
$$


By (83) and the fact that $\mathcal{B}$ is $\approx$-compatible, we get

$$
\begin{aligned}
\pi_{G}(\mathrm{~L}(\mathcal{B})) & =\pi_{G}\left([\mathrm{~L}(\mathcal{B})]_{\approx}\right) \\
& =\pi_{G}([\mathrm{~L}(\mathcal{B}) \cap \operatorname{Red}(H, t)] \approx) \\
& =\pi_{G}(\mathrm{~L}(\mathcal{B}) \cap \operatorname{Red}(H, t)) \\
& =\pi_{G}\left(\pi_{G}^{-1}(K) \cap \operatorname{Red}(H, t)\right) \\
& =K,
\end{aligned}
$$

and thus,

$$
\pi_{G^{\prime}}^{-1}\left(\pi^{\prime}(K)\right)=\pi_{G^{\prime}}^{-1}\left(\pi^{\prime}\left(\pi_{G}(\mathrm{~L}(\mathcal{B}))\right)\right) .
$$

The commutative diagram in Figure 1 yields

$$
\pi_{G^{\prime}}^{-1}\left(\pi^{\prime}(K)\right)=\pi_{G^{\prime}}^{-1}\left(\pi_{G^{\prime}}\left(\pi_{*}^{\prime}(\mathrm{L}(\mathcal{B}))\right)\right)=\left[\pi_{*}^{\prime}(\mathrm{L}(\mathcal{B}))\right]_{\approx^{\prime}}=\left[\mathrm{L}\left(\pi_{*}^{\prime}(\mathcal{B})\right)\right]_{\approx^{\prime}} .
$$

By Claim 46 and $(90), \pi_{*}^{\prime}(\mathcal{B})$ is $d$-closed and hence $\approx^{\prime}$-compatible by Lemma 45 . Hence,

$$
\begin{aligned}
\pi_{G^{\prime}}^{-1}\left(\pi^{\prime}(K)\right) \cap \operatorname{Red}\left(H^{\prime}, t\right) & =\left[\mathrm{L}\left(\pi_{*}^{\prime}(\mathcal{B})\right)\right]_{\approx^{\prime}} \cap \operatorname{Red}\left(H^{\prime}, t\right) \\
& =\left[\mathrm{L}\left(\pi_{*}^{\prime}(\mathcal{B})\right) \cap \operatorname{Red}\left(H^{\prime}, t\right)\right]_{\approx^{\prime}} \cap \operatorname{Red}\left(H^{\prime}, t\right) \\
& =\left[\mathrm{L}\left(\pi_{*}^{\prime}(\mathcal{B})\right) \cap \operatorname{Red}\left(H^{\prime}, t\right)\right]_{\sim^{\prime}} \\
& =\mathrm{L}\left(\pi_{*}^{\prime}(\mathcal{B})\right) \cap \operatorname{Red}\left(H^{\prime}, t\right),
\end{aligned}
$$

where the last equality holds since $\pi_{*}^{\prime}(\mathcal{B})$ is $\sim^{\prime}$-saturated by Lemma 21 . Together with (94) and (95) we get

$$
s^{\prime} \notin \pi_{G^{\prime}}^{-1}\left(\pi^{\prime}(K)\right) .
$$

This shows that $\pi_{G^{\prime}}\left(s^{\prime}\right) \notin \pi^{\prime}(K)$, i.e., by $(93), \pi_{G^{\prime}}\left(\pi_{*}^{\prime}(s)\right) \notin \pi^{\prime}(K)$. The commutative diagram in Figure 1 implies $\pi^{\prime}\left(\pi_{G}(s)\right) \notin \pi^{\prime}(K)$, i.e., by (87),

$$
\pi^{\prime}(g) \notin \pi^{\prime}(K) .
$$

The group $G^{\prime}$ is the fundamental group of a graph of groups (with just one vertex and one edge) with finite vertex groups (namely $H / N$ ), hence it is a virtually free group, i.e., it has a free subgroup of finite index, see e.g. [DD90, Corollary IV.1.9, p.104]. By the result of [Hal49] every free group is LERF and by [Sco78, Lemma 1.1, p. 556] every group with a finite index subgroup which is LERF is itself LERF. Hence $G^{\prime}$ is LERF. In particular, (97) implies the existence of a finite group $G^{\prime \prime}$ and a homomorphism $\pi^{\prime \prime}: G^{\prime} \rightarrow G^{\prime \prime}$ such that

$$
\pi^{\prime \prime}\left(\pi^{\prime}(g)\right) \notin \pi^{\prime \prime}\left(\pi^{\prime}(K)\right) .
$$

By Remark 4, this proves that $G$ is LERF. 


\section{Amalgamated free products}

In the rest of this paper, we will prove for amalgamated free products results similar to those for HNN-extensions from Section 4.

Let us consider two monoids $H_{1}$ and $H_{2}$, two finite subgroups $A_{1} \leq H_{1}, A_{2} \leq H_{2}$, and an isomorphism $\varphi: A_{1} \rightarrow A_{2}$. The corresponding amalgamated free product

$$
G=\left\langle H_{1} * H_{2} ; a=\varphi(a)\left(a \in A_{1}\right)\right\rangle
$$

is defined by $G=\left(H_{1} * H_{2}\right) / \approx$, where $\approx$ is the congruence on the free product $H_{1} * H_{2}$ generated by the equations $a=\varphi(a)$ for $a \in A_{1}$. An $\left(H_{1}, H_{2}\right)$-sequence is an element $s \in H_{1} * H_{2}$; it has a unique decomposition of the form

$$
s=h_{0} k_{1} h_{1} \cdots k_{i} h_{i} \cdots k_{n} h_{n},
$$

where $n \geq 0, h_{1}, \ldots, h_{n-1} \in H_{2} \backslash 1, k_{1}, \ldots, k_{n} \in H_{1} \backslash 1$, and $h_{0}, h_{n} \in H_{2}$. A reduced $\left(H_{1}, H_{2}\right)$-sequence is an element $s \in H_{1} * H_{2}$ of the form

$$
s=h_{0} k_{1} h_{1} \cdots k_{i} h_{i} \cdots k_{n} h_{n},
$$

where $n \geq 0, h_{1}, \ldots, h_{n-1} \in H_{2} \backslash A_{2}, k_{1}, \ldots, k_{n} \in H_{1} \backslash A_{1}$ and $h_{0}, h_{n} \in H_{2}$. We denote by $\operatorname{Red}\left(H_{1}, H_{2}\right)$ the set of all reduced $\left(H_{1}, H_{2}\right)$-sequences.

Assume now that $H_{1}=X_{1}^{*} / \equiv_{H_{1}}$ and $H_{2}=X_{2}^{*} / \equiv_{H_{2}}$ for generating sets $X_{1}$ and $X_{2}$ such that $X_{1} \cap X_{2}=\emptyset$. Then $G=\left(X_{1} \cup X_{2}\right)^{*} / \equiv$ where $\equiv$ is the smallest congruence over the free monoid $\left(X_{1} \cup X_{2}\right)^{*}$ such that $\left(\equiv_{H_{1}} \cup \equiv_{H_{2}}\right) \subseteq \equiv$ and $a \equiv \varphi(a)$ for all $a \in A_{1}$. Let

$$
\pi:\left(X_{1} \cup X_{2}\right)^{*} \rightarrow G
$$

denote the canonical morphism defined by $\pi(w)=[w] \equiv$ for $w \in\left(X_{1} \cup X_{2}\right)^{*}$. This morphism naturally factorizes as

$$
\pi=\pi_{a} \circ \pi_{G}
$$

where

$$
\begin{aligned}
& \pi_{a}:\left(X_{1} \cup X_{2}\right)^{*} \rightarrow H_{1} * H_{2} \text { and } \\
& \pi_{G}: H_{1} * H_{2} \rightarrow G .
\end{aligned}
$$

The kernel of $\pi_{G}$ coincides with the congruence $\approx$ over $H_{1} * H_{2}$ defined above.

We define the binary relation $\stackrel{1}{\sim}$ over $H_{1} * H_{2}$ as follows: For all $s, s^{\prime} \in H_{1} * H_{2}$ let $s \stackrel{1}{\sim} s^{\prime}$ if and only if there are $\left(H_{1}, H_{2}\right)$-sequences $s_{1}$ and $s_{2}$ and $a, b \in A_{1}$ such that either

$$
\begin{aligned}
& \exists h \in H_{1}: s=s_{1} h s_{2} \wedge s^{\prime}=s_{1} \varphi(a)\left(a^{-1} h b\right) \varphi\left(b^{-1}\right) s_{2} \quad \text { or } \\
& \exists h \in H_{2}: s=s_{1} h s_{2} \wedge s^{\prime}=s_{1} a\left(\varphi\left(a^{-1}\right) h \varphi(b)\right) b^{-1} s_{2} .
\end{aligned}
$$

Let us denote by $\sim \subseteq \approx$ the congruence over $H_{1} * H_{2}$ generated by $\stackrel{1}{\sim} ;$ it coincides with the reflexive and transitive closure of $\stackrel{1}{\sim}$. Equivalently, if

$$
\begin{aligned}
s & =h_{0} k_{1} h_{1} k_{2} \cdots h_{n-1} k_{n} h_{n} \text { and } \\
s^{\prime} & =h_{0}^{\prime} k_{1}^{\prime} h_{1}^{\prime} k_{2}^{\prime} \cdots h_{m-1}^{\prime} k_{m}^{\prime} h_{m}^{\prime}
\end{aligned}
$$


(with $n, m \geq 0$, and $k_{1}, \ldots, k_{n}, k_{1}^{\prime}, \ldots, k_{m}^{\prime} \in H_{1}, h_{0}, \ldots, h_{n}, h_{0}^{\prime}, \ldots, h_{m}^{\prime} \in H_{2}$ ) are reduced sequences, then $s \sim s^{\prime}$ if and only if $n=m$ and there exist $a_{1}, \ldots, a_{2 n} \in A_{1}$ such that:

- $h_{i} \varphi\left(a_{2 i+1}\right)=\varphi\left(a_{2 i}\right) h_{i}^{\prime}$ in $H_{2}$ for $0 \leq i \leq n$ (here we set $a_{0}=a_{2 n+1}=1$ )

- $k_{i} a_{2 i}=a_{2 i-1} k_{i}^{\prime}$ in $H_{1}$ for $1 \leq i \leq n$

In other words, there exists a Van Kampen diagram of the following kind, where $n=m=4$. Here, we omit the $\varphi\left(a_{i}\right)$-labelled edge, which runs parallel to the $a_{i}$-labelled vertical edge $(1 \leq i \leq 2 n){ }^{\mathrm{c}}$ Light-shaded (resp. dark-shaded) areas represent relation in $H_{2}$ (resp. relations in $H_{1}$ ).

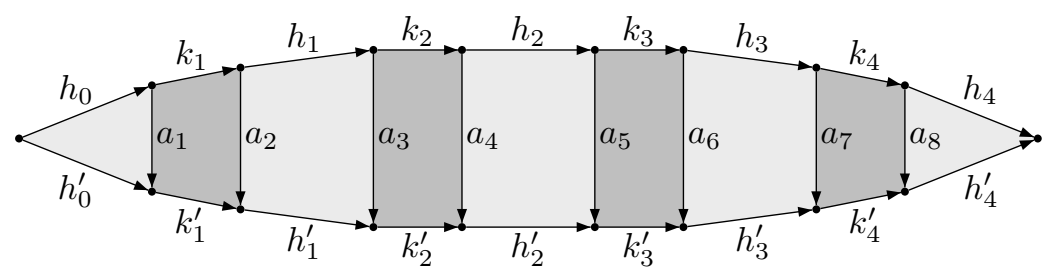

The set $\operatorname{Red}\left(H_{1}, H_{2}\right)$ is again saturated by the congruence $\sim$, i.e., $s \sim s^{\prime}$ implies $s \in \operatorname{Red}\left(H_{1}, H_{2}\right) \Leftrightarrow s^{\prime} \in \operatorname{Red}\left(H_{1}, H_{2}\right)$. Here it is important that $A_{1}$ and $A_{2}$ are groups.

For the case that $H_{1}$ and $H_{2}$ (and hence $G$ ) are groups, it is well-known that $G$ can be embedded into the HNN-extension

$$
\widehat{G}=\left\langle H_{1} * H_{2}, t ; t^{-1} a t=\varphi(a)\left(a \in A_{1}\right)\right\rangle
$$

by the map $\Phi$ with

$$
\Phi: k \mapsto t^{-1} k t \text { for } k \in H_{1} \text { and } h \mapsto h \text { for } h \in H_{2},
$$

see e.g. [LS77, Theorem 2.6, p. 187]. This result generalizes to our situation, where $H_{1}$ and $H_{2}$ are monoids but $A_{1}$ and $A_{2}$ are groups: Assume that $s_{1}, s_{2} \in$ $\operatorname{Red}\left(H_{1}, H_{2}\right)$ such that $\Phi(s) \approx \Phi\left(s^{\prime}\right)$. Then $\Phi(s), \Phi\left(s^{\prime}\right) \in \operatorname{Red}(H, t)$, and Lemma 5 implies $\Phi(s) \sim \Phi\left(s^{\prime}\right)$. The defining relations of $\sim$ imply $s \sim s^{\prime}$.

With our definitions of the relations $\approx$ and $\sim$ on the free product $H_{1} * H_{2}$ and the new definition of a reduced sequence, Lemma 5 again holds:

Lemma 47. Let $s, s^{\prime} \in \operatorname{Red}\left(H_{1}, H_{2}\right)$. Then $s \approx s^{\prime}$ if and only if $s \sim s^{\prime}$.

Proof. Assume that $s \approx s^{\prime}$ for $s, s^{\prime} \in \operatorname{Red}\left(H_{1}, H_{2}\right)$. The defining relations for $\approx$ imply $\Phi(s) \approx \Phi\left(s^{\prime}\right)$. Then, the above arguments yield $s \sim s^{\prime}$.

${ }^{\mathrm{c}}$ Alternatively, one may assume that that $A_{1}=A_{2}=A, H_{1} \cap H_{2}=A$, where $A$ is a subgroup of $H_{1}$ and $H_{2}$, and that $\varphi$ is the identity map. Then one obtains exactly the above Van Kampen diagram. 


\section{Finite automata for amalgamated free products}

We fix throughout this section two monoids $H_{1}, H_{2}$, two finite subgroups $A_{1} \leq$ $H_{1}, A_{2} \leq H_{2}$, an isomorphism $\varphi: A_{1} \rightarrow A_{2}$ and the corresponding amalgamated free product

$$
G=\left\langle H_{1} * H_{2} ; a=\varphi(a)\left(a \in A_{1}\right)\right\rangle .
$$

\subsection{Finite $\left(\mathrm{H}_{1}, \mathrm{H}_{2}\right)$-automata}

The classical embedding (102) leads naturally to the following adaptations of the definitions from Section 4 to the case of an amalgamated free product.

Let $\mathcal{F}_{i} \subseteq \mathcal{P}\left(H_{i}\right)$ be a set of subsets of $H_{i}(i \in\{1,2\})$ such that every singleton $\{a\}$ with $a \in A_{i}$ belongs to $\mathcal{F}_{i}$. A finite $\left(H_{1}, H_{2}\right)$-automaton over $H_{1} * H_{2}$, with labelling sets $\left(\mathcal{F}_{1}, \mathcal{F}_{2}\right)$ is a 5 -tuple

$$
\mathcal{A}=\langle\mathcal{L}, \mathrm{Q}, \delta, \mathrm{I}, \mathrm{T}\rangle
$$

where $\mathcal{L}$ is finite and

$$
\mathcal{L} \subseteq \mathcal{F}_{1} \cup \mathcal{F}_{2},
$$

$\mathrm{Q}$ is a finite set of states, $\mathrm{I} \subseteq \mathrm{Q}$ is the set of initial states, $\mathrm{T} \subseteq \mathrm{Q}$ is the set of terminal states, and $\delta$ (the set of transitions) is a subset of $\mathrm{Q} \times \mathcal{L} \times \mathrm{Q}$ such that for every state $q \in \mathrm{Q}$ there exists $L \in \mathcal{L}$ with $(q, L, q) \in \delta$ and $1 \in L$. For the last point, 1 denotes both the neutral element of $H_{1}$ and $H_{2}$, The representation map $\mu_{\mathcal{A}}: H_{1} * H_{2} \rightarrow \mathrm{BR}(\mathrm{Q})$ is defined as follows. First define for every $h \in H_{1} \cup H_{2}$ :

$$
\mu_{\mathcal{A}, 0}(h)=\{(q, r) \in Q \times Q \mid \exists L \in \mathcal{L}:(q, L, r) \in \delta \wedge h \in L\} .
$$

Now, for every $s \in H_{1} * H_{2}$ of the form (100), let

$$
\mu_{\mathcal{A}}(s)=\mu_{\mathcal{A}, 0}\left(h_{0}\right) \circ \mu_{\mathcal{A}, 0}\left(k_{1}\right) \circ \mu_{\mathcal{A}, 0}\left(h_{1}\right) \circ \cdots \circ \mu_{\mathcal{A}, 0}\left(k_{n}\right) \circ \mu_{\mathcal{A}, 0}\left(h_{n}\right) .
$$

The set $\mathrm{L}(\mathcal{A}) \subseteq H_{1} * H_{2}$ recognized by $\mathcal{A}$ is defined as for HNN-extensions.

Let us introduce a finite set $\mathcal{T}_{4}$ of 4 types, which will induce a partition of the set of states of a finite $\left(H_{1}, H_{2}\right)$-automaton:

$$
\mathcal{T}_{4}=\left\{\left(A_{1}, H_{1}\right),\left(A_{2}, H_{2}\right),\left(1, H_{2}\right),(1,1)\right\} .
$$

We define a graph $\mathcal{G}_{4}=\left(\mathcal{T}_{4}, \mathcal{E}_{4}\right)$ by:

$$
\begin{aligned}
\mathcal{E}_{4}= & \left\{((C, D), C,(C, D)) \mid(C, D) \in \mathcal{T}_{4}\right\} \\
& \cup\left\{\left(\left(A_{i}, H_{i}\right), H_{i},\left(A_{3-i}, H_{3-i}\right)\right) \mid i \in\{1,2\}\right\} \\
& \cup\left\{\left(\left(A_{2}, H_{2}\right), H_{2},(1,1)\right),\left(\left(1, H_{2}\right), H_{2},\left(A_{1}, H_{1}\right)\right),\left(\left(1, H_{2}\right), H_{2},(1,1)\right)\right\} .
\end{aligned}
$$

The graph $\mathcal{G}_{4}$ is represented in Figure 2. An analogous graph $\mathcal{R}_{4}=\left(\mathcal{T}_{4}, \mathcal{E}_{4}^{\prime}\right)$ is defined by

$$
\begin{aligned}
\mathcal{E}_{4}^{\prime}= & \mathcal{E}_{4} \backslash\left\{\left(\left(A_{1}, H_{1}\right), H_{1},\left(A_{2}, H_{2}\right)\right),\left(\left(A_{2}, H_{2}\right), H_{2},\left(A_{1}, H_{1}\right)\right)\right\} \\
& \cup\left\{\left(\left(A_{1}, H_{1}\right), H_{1} \backslash A_{1},\left(A_{2}, H_{2}\right)\right),\left(\left(A_{2}, H_{2}\right), H_{2} \backslash A_{2},\left(A_{1}, H_{1}\right)\right)\right\},
\end{aligned}
$$



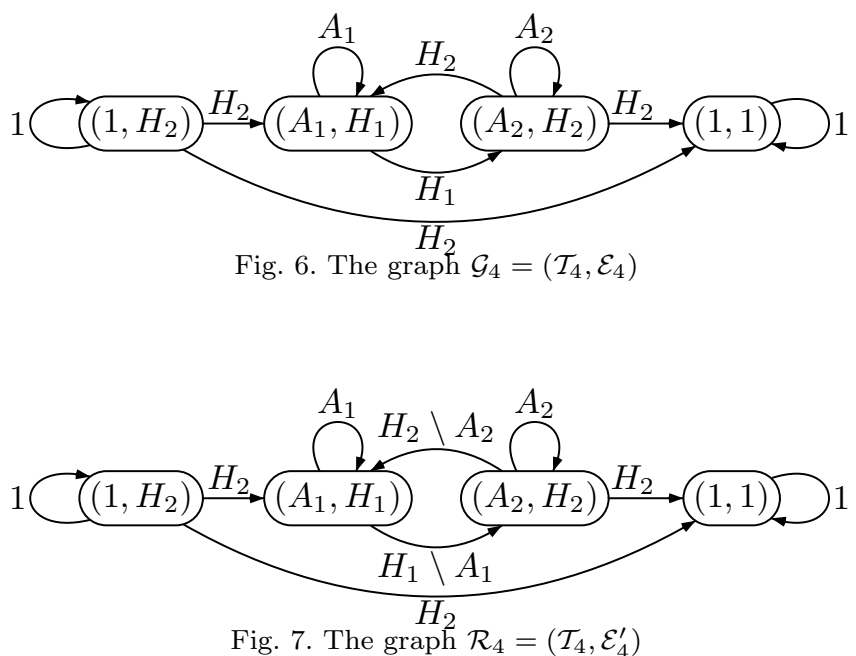

see Figure 7 . The set $\widehat{\mathcal{E}}_{4}$ is defined analogously to $\widehat{\mathcal{E}}_{6}$. The reader might be surprised by the asymmetry in the definition of $\mathcal{G}_{4}$ and $\mathcal{R}_{4}$ : it corresponds to our choice for the definition of an $\left(H_{1}, H_{2}\right)$-sequence in (100), where we require $h_{0}, h_{n} \in H_{2}$. This choice was made arbitrarily; we could have also required $h_{0}, h_{n} \in H_{1}$.

A partitioned finite $\left(H_{1}, H_{2}\right)$-automaton with labelling sets $\left(\mathcal{F}_{1}, \mathcal{F}_{2}\right)$ is a 6 -tuple

$$
\mathcal{A}=\langle\mathcal{L}, \mathrm{Q}, \tau, \delta, \mathrm{I}, \mathrm{T}\rangle,
$$

where $\mathcal{L}, \mathbf{Q}, \delta, \mathbf{I}$, and $\mathrm{T}$ are as above and $\tau: \mathbf{Q} \rightarrow \mathcal{T}_{4}$ assigns a type with every state such that:

$$
\begin{aligned}
& \forall(q, L, r) \in \delta:\{q\} \times L \times\{r\} \subseteq \widehat{\mathcal{E}}_{4} \\
& \tau(\mathrm{I})=\left\{\left(1, H_{2}\right)\right\} \wedge \tau(\mathrm{T})=\{(1,1)\}
\end{aligned}
$$

Let us consider the equivalences $\sim$ and $\approx$ over $H_{1} * H_{2}$ defined in Section 5. The notions of $\sim$-saturated and $\approx$-compatible finite $\left(H_{1}, H_{2}\right)$-automata are defined as in Definition 9 , but with the equivalences $\approx$ and $\sim$ being those defined over $H_{1} * H_{2}$.

The notion of a deterministic and partitioned finite $\left(H_{1}, H_{2}\right)$-automaton $\mathcal{A}$ with labelling sets $\left(\mathcal{F}_{1}, \mathcal{F}_{2}\right)$ is still defined by the properties (33) and (34). For every $q \in \mathrm{Q}$, and $\Theta \in \mathcal{T}_{4}$ we again define the set $\mathrm{L}^{1}(q, \Theta) \subseteq H_{1} \cup H_{2}$ by (35). Then, a partitioned finite $\left(H_{1}, H_{2}\right)$-automaton $\mathcal{A}$ is complete if

$$
\forall\left(\Theta, C, \Theta^{\prime}\right) \in \mathcal{E}_{4}^{\prime} \forall q \in \tau^{-1}(\Theta): C \subseteq \mathrm{L}^{1}\left(q, \Theta^{\prime}\right) .
$$

\subsection{Normalization of automata}

The following three propositions can be now proved in exactly the same way as the corresponding statements for HNN-extensions (Propositions 22, 28 and 33). 
Proposition 48. From a given partitioned and $\approx$-compatible finite $\left(H_{1}, H_{2}\right)$ automaton $\mathcal{A}$ with labelling sets $\left(\mathcal{F}_{1}, \mathcal{F}_{2}\right)$ one can effectively construct a partitioned, $\approx$-compatible, $\sim$-saturated, and unitary $\left(H_{1}, H_{2}\right)$-automaton $\mathcal{B}$ with labelling sets $\left(\left\{a F a^{\prime} \mid F \in \mathcal{F}_{1}, a, a^{\prime} \in A_{1}\right\},\left\{a F a^{\prime} \mid F \in \mathcal{F}_{2}, a, a^{\prime} \in A_{2}\right\}\right)$ and such that $[\mathrm{L}(\mathcal{A})]_{\approx} \cap \operatorname{Red}\left(H_{1}, H_{2}\right)=\mathrm{L}(\mathcal{B}) \cap \operatorname{Red}\left(H_{1}, H_{2}\right)$.

Proposition 49. From a given partitioned, $\approx$-compatible, and $\sim$-saturated finite $\left(H_{1}, H_{2}\right)$-automaton $\mathcal{B}$ with labelling sets $\left(\mathcal{F}_{1}, \mathcal{F}_{2}\right)$ one can effectively construct a partitioned, $\sim$-saturated, deterministic, and complete finite $\left(H_{1}, H_{2}\right)$-automaton $\mathcal{C}$ with labelling sets $\left(\operatorname{Bool}\left(\mathcal{F}_{1}\right), \operatorname{Bool}\left(\mathcal{F}_{2}\right)\right)$ and such that $[\mathrm{L}(\mathcal{A})]_{\approx} \cap \operatorname{Red}\left(H_{1}, H_{2}\right)=$ $\mathrm{L}(\mathcal{C})$.

Proposition 50. Let $K \subseteq G$. The following are equivalent:

(1) $K$ is a rational subset of $G$.

(2) $K=\pi(R)$ for some rational subset $R \subseteq\left(X_{1} \cup X_{2}\right)^{*}$.

(3) $\pi_{G}^{-1}(K) \cap \operatorname{Red}\left(H_{1}, H_{2}\right)=\mathrm{L}(\mathcal{B}) \cap \operatorname{Red}\left(H_{1}, H_{2}\right)$ for some partitioned, $\approx$ compatible, and $\sim$-saturated finite $\left(H_{1}, H_{2}\right)$-automaton $\mathcal{B}$ with labelling sets $\left(\operatorname{Rat}\left(H_{1}\right), \operatorname{Rat}\left(H_{2}\right)\right)$.

Moreover, in point (3), if the membership problem for languages in $\operatorname{Rat}\left(H_{1}\right)$ and $\operatorname{Rat}\left(H_{2}\right)$, respectively, is decidable, then $\mathcal{B}$ can be computed effectively from a finite automaton for $R$ in point (2).

\subsection{Transfer results for rational sets}

Using Propositions 48, 49 and 50, the following transfer results for amalgamated free products can be shown in exactly the same way as for HNN-extensions.

Theorem 51. Let $G=\left\langle H_{1} * H_{2} ; a=\varphi(a)\left(a \in A_{1}\right)\right\rangle$, where $H_{1}$ and $H_{2}$ are countably-generated monoids with subgroups $A_{1} \leq H_{1}$ and $A_{2} \subseteq H_{2}$ and $\varphi: A_{1} \rightarrow$ $A_{2}$ is an isomorphism. Then we have:

(1) If $\operatorname{Rat}\left(H_{1}\right)$ and $\operatorname{Rat}\left(H_{2}\right)$ are closed under intersection, then also $\operatorname{Rat}(G)$ is closed under intersection.

(2) If $\operatorname{Rat}\left(H_{1}\right)$ and $\operatorname{Rat}\left(H_{2}\right)$ are closed under complement, then also $\operatorname{Rat}(G)$ is closed under complement.

(3) If the membership problem is decidable for languages in $\operatorname{Rat}\left(H_{1}\right)$ and $\operatorname{Rat}\left(H_{2}\right)$, then the same is true for languages in $\operatorname{Rat}(G)$.

(4) If the emptiness problem is decidable for languages in the boolean closure of $\operatorname{Rat}\left(H_{1}\right)$ and $\operatorname{Rat}\left(H_{2}\right)$, then the same is true for languages in the boolean closure of $\operatorname{Rat}(G)$.

Statement (3) is covered by [KSS06, Theorem 5.1] (for free products it was shown in [Ned00]) for the case that $H_{1}$ and $H_{2}$ (and hence $G$ ) are groups, whereas (1), (2), and (4) are new to the knowledge of the authors. 


\subsection{Transfer results for finitely generated subgroups}

Let us assume in this section that $H_{1}$ and $H_{2}$ (and hence $G$ in (99)) are groups. The following subsets $S_{1} \subseteq \mathcal{P}\left(H_{1}\right)$ and $S_{2} \subseteq \mathcal{P}\left(H_{2}\right)$ serve for labelling finite $\left(H_{1}, H_{2}\right)$ automata:

$$
\begin{aligned}
& \mathcal{S}_{1}=\left\{K h \mid K \text { is a finitely generated subgroup of } H_{1}, h \in H_{1}\right\} \\
& \mathcal{S}_{2}=\left\{K h \mid K \text { is a finitely generated subgroup of } H_{2}, h \in H_{2}\right\}
\end{aligned}
$$

Proposition 52. Let $K$ be a subgroup of $G$. The following are equivalent:

(1) $K$ is finitely generated.

(2) $K$ is rational.

(3) There exists some finite automaton $\mathcal{A}$ over the free monoid $\left(X_{1} \cup X_{2}\right)^{*}$ such that $K=\pi(\mathrm{L}(\mathcal{A}))$.

(4) $\pi_{G}^{-1}(K) \cap \operatorname{Red}\left(H_{1}, H_{2}\right)=\mathrm{L}(\mathcal{B}) \cap \operatorname{Red}\left(H_{1}, H_{2}\right)$ for some partitioned, $\approx$ compatible, and $\sim$-saturated finite $\left(H_{1}, H_{2}\right)$-automaton $\mathcal{B}$ with labelling sets $\left(\mathcal{S}_{1}, \mathcal{S}_{2}\right)$.

Moreover, in (4), if the generalized word problem for $\mathrm{H}_{1}$ and $\mathrm{H}_{2}$ is decidable, then $\mathcal{B}$ can be constructed effectively from $\mathcal{A}$ in (3).

Theorem 53. Let $G=\left\langle H_{1} * H_{2} ; a=\varphi(a)\left(a \in A_{1}\right)\right\rangle$, where $H_{1}$ and $H_{2}$ are countably-generated groups with subgroups $A_{1} \leq H_{1}$ and $A_{2} \subseteq H_{2}$ and $\varphi: A_{1} \rightarrow A_{2}$ is an isomorphism. Then we have:

(1) If $H_{1}$ and $H_{2}$ have a decidable generalized word problem, then also $G$ has a decidable generalized word problem.

(2) If $H_{1}$ and $H_{2}$ have the Howson property, then also $G$ has the Howson property.

(3) If $H_{1}$ and $H_{2}$ have the LERF property, then also $G$ has the LERF property.

Statement (1) is covered by [KWM05, Corollary 5.15], statement (2) was shown in [Bez98,KS70], and statement (3) was shown in [AG73].

Remark 54. There is an alternative way to deduce Theorem 53: using the embedding (102) together with the Theorem 43 on HNN-extensions it suffices to prove that (i) decidability of the generalized word problem, (ii) the Howson property, and (iii) the LERF property are preserved under (i) passing to finitely generated subgroups and (ii) free products. Preservation under passing to finitely generated subgroups is obvious. Preservation under free products can be proved using automata theoretic constructions, which are similar but much simpler than our constructions for amalgamated free products.

For Theorem 51 about rational subsets, the same line of arguments can be applied to the membership problem for rational subsets and the emptiness problem for boolean combinations of rational subsets (decidability of these problems is clearly preserved under passing to finitely generated submonoids). On the other hand, it not clear whether these arguments also apply to the closure under intersection and 
complement, respectively, because it is not clear whether these closure properties are preserved under passing to finitely generated submonoids.

Acknowledgments We would like to thank Volker Diekert, Benjamin Steinberg, and the anonymous referee for their useful comments.

\section{References}

[AG73] R.B.J.T. Allenby and R.J. Gregorac. On locally extended residually finite groups. In Conference on Group Theory (Univ. Wisconsin-Parkside, Kenosha, Wis., 1972), number 319 in Lecture Notes in Mathematics, pages 9-17. Springer, 1973.

[AH89] IJ. Jan Aalbersberg and H.J. Hoogeboom. Characterizations of the decidability of some problems for regular trace languages. Math. Systems Theory, 22(1):119, 1989.

[AS75] A.W. Anissimow and F.D. Seifert. Zur algebraischen Charakteristik der durch kontext-freie Sprachen definierten Gruppen. Elektron. Informationsverarbeit. Kybernetik, 11(10-12):695-702, 1975.

[Ben69] M. Benois. Parties rationnelles du groupe libre. C. R. Acad. Sci. Paris, Sér. A, 269:1188-1190, 1969.

[Bez98] V.N. Bezverkhniı̌. On the intersection of subgroups in HNN-groups. Fundam. Prikl. Mat., 4(1):199-222, 1998.

[BJW82] R.V. Book, M. Jantzen, and C. Wrathall. Monadic Thue systems. Theoret. Comput. Sci., 19(3):231-251, 1982.

[DJ91] N. Dershowitz and J.P. Jouannaud. Rewrite systems. In Handbook of theoretical computer science, vol.B, Chapter 2, pages 243-320. Elsevier, 1991.

[DD90] W. Dicks and M.J. Dunwoody. Groups acting on graphs. Cambridge University Press, 1990.

[Die05] V. Diekert. Personal communication. 2005.

[Git97] R. Gitik. Graphs and separability properties of groups. J. Algebra, 188(1):125143, 1997.

[Gru90] Z. Grunschlag. Algorithms in Geometric Group Theory. PhD thesis, University of California at Berkeley, 1990.

[Hal49] M. Hall, Jr. Coset representations in free groups. Trans. Amer. Math. Soc., 67:421-432, 1949.

[HNN49] G. Higman, B.H. Neumann, and H. Neumann. Embedding theorems for groups. J. London Math. Soc., 24:247-254, 1949.

[How63] J. M. Howie. Embedding theorems for semigroups. The Quarterly Journal of Mathematics. Oxford. Second Series, 14:254-258, 1963.

[KSS06] M. Kambites, P.V. Silva, and B. Steinberg. On the rational subset problem for groups. J. Algebra, 309(2):622-639, 2007.

[KWM05] I. Kapovich, R. Weidmann, and A. Myasnikov. Foldings, graphs of groups and the membership problem. Internat. J. Algebra Comput., 15(1):95-128, 2005.

[KS70] A. Karrass and D. Solitar. The subgroups of a free product of two groups with an amalgamated subgroup. Trans. Amer. Math. Soc., 150:227-255, 1970.

[KS71] A. Karrass and D. Solitar. Subgroups of HNN groups and groups with one defining relation. Canad. J. Math., 23:627-643, 1971.

[LS77] R.C. Lyndon and P.E. Schupp. Combinatorial group theory. Springer-Verlag, Berlin, 1977. Ergebnisse der Mathematik und ihrer Grenzgebiete, Band 89. 
[LS06a] M. Lohrey and G. Sénizergues. Equations in HNN-extensions. Manuscript, 2006.

[LS06b] M. Lohrey and G. Sénizergues. Positive theories of HNN-extensions and amalgamated free products. Manuscript, 2006.

[ME07a] L. Markus-Epstein. Algorithmic problems in amalgams of finite groups. Technical report, arXiv.org, 2007. http://arxiv.org/abs/0705.0759.

[ME07b] L. Markus-Epstein. Stallings' foldings and subgroups of amalgams of finite groups. Technical report, arXiv.org, 2007. http://arxiv.org/abs/0705.0754.

[MR04] V. Metaftsis and E. Raptis. Subgroup separability of graphs of abelian groups. Proc. Amer. Math. Soc., 132(7):1873-1884, 2004.

[Ned00] M.Yu. Nedbaj. The occurrence problem in a rational subset of the free product of groups. Vestn. Omsk. Univ., 2000(2):17-18, 2000.

[Reu85] Ch. Reutenauer. Sur les semi-groupes vérifiant le théorème de Kleene. RAIRO Inform. Théor., 19(3):281-291, 1985.

[Sak87] J. Sakarovitch. On regular trace languages. Theoret. Comput. Sci., 52:59-75, 1987.

[Sak92] J. Sakarovitch. The "last" decision problem for rational trace languages. In Proceedings of the 1st Latin American Symposium on Theoretical Informatics (LATIN'92), number 583 in Lecture Notes in Computer Science, pages 460473. Springer, 1992.

[Sco78] P. Scott. Subgroups of surface groups are almost geometric. J. London Math. Soc. (2), 17(3):555-565, 1978.

[Sén94] G. Sénizergues. Formal languages and word-rewriting. In Term Rewriting, Font-Romeu 93, advanced course, number 909 in Lecture Notes in Computer Science, pages 75-94. Springer, 1994.

[Sén96] G. Sénizergues. On the rational subsets of the free group. Acta Inform., 33(3):281-296, 1996.

[Ser03] J.-P. Serre. Trees. Springer Monographs in Mathematics. Springer-Verlag, Berlin, 2003. Translated from the French original by J. Stillwell, Corrected 2nd printing of the 1980 English translation.

[Sta71] J.R. Stallings. Group Theory and Three-Dimensional Manifolds. number 4 in Yale Mathematical Monographs. Yale University Press, 1971.

[Sta83] John R. Stallings. Topology of finite graphs. Invent. Math., 71(3):551-565, 1983.

[Wis00] D. T. Wise. Subgroup separability of graphs of free groups with cyclic edge groups. Q. J. Math., 51(1):107-129, 2000.

\section{Appendix A. Normal forms}

We check here that the classical notion of normal form for HNN-extensions of groups adapts to the case of monoids, provided that $A$ and $B$ are subgroups of the base monoid. This will result in a proof of Lemma 5 .

Let us fix a monoid $H$, isomorphic subgroups $A$ and $B$ of $H$, and an isomorphism $\varphi: A \rightarrow B$, as considered in Section 3 and let $G$ be the corresponding HNNextension defined by (7).

For every subgroup $C$ of $H$, the left-congruence $\equiv_{C}$ is defined by

$$
h \equiv_{C} h^{\prime} \Leftrightarrow \exists c \in C: h=h^{\prime} c .
$$

Since $C$ is a group, $\equiv_{C}$ is indeed an equivalence relation. The equivalence classes 
of $\equiv_{C}$ are the sets $h C$ for $h \in H$. Let us choose some systems of representatives $R_{A}$ (resp. $R_{B}$ ) for the equivalence $\equiv_{A}\left(\right.$ resp. $\left.\equiv_{B}\right)$. We suppose that $1 \in R_{A} \cap R_{B}$. Hence, $A \cap R_{A}=\{1\}=B \cap R_{B}$. We define now the set of normal forms.

Definition 55. A normal form is an element of $H *\left\{t, t^{-1}\right\}^{*}$ of the form

$$
s=h_{0} t^{\alpha_{1}} h_{1} \cdots t^{\alpha_{i}} h_{i} \cdots t^{\alpha_{n}} h_{n},
$$

where $n \geq 0$, such that, for every $i \in\{1, \ldots, n\}$

(i) $h_{n}$ is an arbitrary element of $H$,

(ii) if $\alpha_{i}=1$ then $h_{i-1} \in R_{A}$,

(iii) if $\alpha_{i}=-1$ then $h_{i-1} \in R_{B}$, and

(iv) if $\alpha_{i}=-\alpha_{i+1}$ then $h_{i} \neq 1$.

One can notice that, due to conditions (ii)-(iv), every normal form is a reduced sequence. We denote by $\mathcal{N}$ the set of all normal forms. We define a right-action $\odot$ of the monoid $H *\left\{t, t^{-1}\right\}^{*}$ over $\mathcal{N}$ as follows. Let $s$ be a normal form of the form (A.1). For every $h \in H$ we set

$$
s \odot h=h_{0} t^{\alpha_{1}} h_{1} \cdots t^{\alpha_{i}} h_{i} \cdots t^{\alpha_{n}}\left(h_{n} h\right) .
$$

The right-action of $t$ on $s$ is defined through 3 cases:

Case (t0). $n=0$ and $h_{0}=r_{0} a$ with $r_{0} \in R_{A}$ and $a \in A$ :

$$
s \odot t=\left(r_{0} a\right) \odot t=r_{0} t \varphi(a)
$$

Case (t1). $n>0, \alpha_{n}=1, h_{n}=r_{n} a$ with $r_{n} \in R_{A}, a \in A$ :

$$
s \odot t=h_{0} t^{\alpha_{1}} h_{1} \cdots t^{\alpha_{i}} h_{i} \cdots t^{\alpha_{n}} r_{n} t \varphi(a)
$$

Case (t2). $n>0, \alpha_{n}=-1, h_{n}=r_{n} a$ with $r_{n} \in R_{A} \backslash\{1\}, a \in A$ :

$$
s \odot t=h_{0} t^{\alpha_{1}} h_{1} \cdots t^{\alpha_{i}} h_{i} \cdots t^{\alpha_{n}} r_{n} t \varphi(a)
$$

Case (t3). $n>0, \alpha_{n}=-1, h_{n} \in A$ :

$$
s \odot t=h_{0} t^{\alpha_{1}} h_{1} \cdots t^{\alpha_{i}} h_{i} \cdots t^{\alpha_{n-1}}\left(h_{n-1} \varphi\left(h_{n}\right)\right)
$$

The right-action of $t^{-1}$ on the normal form $s$ is defined similarly through 3 cases:

Case $\left(t^{-1} 0\right) . n=0$

Case ( $\left.t^{-1} 1\right) . n>0, \alpha_{n}=-1, h_{n}=r_{n} b$ with $r_{n} \in R_{B}, b \in B$

Case (t-12). $n>0, \alpha_{n}=1, h_{n}=r_{n} b$ with $r_{n} \in R_{B} \backslash\{1\}, b \in B$

Case (t $\left.{ }^{-1} 3\right) . n>0, \alpha_{n}=1, h_{n} \in B$

One can easily check that for every $s \in \mathcal{N}$ :

$$
s \odot\left(t t^{-1}\right)=s \odot\left(t^{-1} t\right)=s
$$


Let us compute the sequence $s \odot t b t^{-1}$, when $s$ fulfils case $(t 1)$ or $(t 2)$ :

$$
\begin{aligned}
s \odot t b t^{-1} & =\left(h_{0} t^{\alpha_{1}} h_{1} \cdots t^{\alpha_{i}} h_{i} \cdots t^{\alpha_{n}} r_{n} t \varphi(a)\right) \odot\left(b t^{-1}\right) \\
& =\left(h_{0} t^{\alpha_{1}} h_{1} \cdots t^{\alpha_{i}} h_{i} \cdots t^{\alpha_{n}} r_{n} t(\varphi(a) b)\right) \odot t^{-1} \\
& =h_{0} t^{\alpha_{1}} h_{1} \cdots t^{\alpha_{i}} h_{i} \cdots t^{\alpha_{n}}\left(r_{n} \varphi^{-1}(\varphi(a) b)\right) \\
& =h_{0} t^{\alpha_{1}} h_{1} \cdots t^{\alpha_{i}} h_{i} \cdots t^{\alpha_{n}}\left(r_{n} a \varphi^{-1}(b)\right) \\
& =h_{0} t^{\alpha_{1}} h_{1} \cdots t^{\alpha_{i}} h_{i} \cdots t^{\alpha_{n}}\left(h_{n} \varphi^{-1}(b)\right) \\
& =\left(h_{0} t^{\alpha_{1}} h_{1} \cdots t^{\alpha_{i}} h_{i} \cdots t^{\alpha_{n}} h_{n}\right) \odot \varphi^{-1}(b) .
\end{aligned}
$$

When the sequence $s$ fulfils case (t3) we obtain:

$$
\begin{aligned}
s \odot t b t^{-1} & =\left(h_{0} t^{\alpha_{1}} h_{1} \cdots t^{\alpha_{i}} h_{i} \cdots t^{\alpha_{n-1}}\left(h_{n-1} \varphi\left(h_{n}\right)\right)\right) \odot\left(b t^{-1}\right) \\
& =\left(h_{0} t^{\alpha_{1}} h_{1} \cdots t^{\alpha_{i}} h_{i} \cdots t^{\alpha_{n-1}}\left(h_{n-1} \varphi\left(h_{n}\right) b\right)\right) \odot t^{-1} \\
& =h_{0} t^{\alpha_{1}} h_{1} \cdots t^{\alpha_{i}} h_{i} \cdots t^{\alpha_{n-1}} h_{n-1} t^{-1}\left(h_{n} \varphi^{-1}(b)\right) \\
& =\left(h_{0} t^{\alpha_{1}} h_{1} \cdots t^{\alpha_{i}} h_{i} \cdots t^{\alpha_{n}} h_{n}\right) \odot \varphi^{-1}(b)
\end{aligned}
$$

Finally in case $(t 0)$ we have

$$
\begin{aligned}
s \odot t b t^{-1} & =\left(r_{0} a\right) \odot t b t^{-1}=\left(r_{0} t(\varphi(a) b)\right) \odot t^{-1}=r_{0} a \varphi^{-1}(b) \\
& =s \odot \varphi^{-1}(b) .
\end{aligned}
$$

We have thus established that for every $s \in \mathcal{N}$ :

$$
s \odot\left(t b t^{-1}\right)=s \odot \varphi^{-1}(b)
$$

The set of relations $\left\{\left(t t^{-1}, 1\right),\left(t^{-1} t, 1\right)\right\} \cup\left\{\left(t b t^{-1}, \varphi^{-1}(b)\right) \mid b \in B\right\}$ generates the congruence $\approx$ over $H *\left\{t, t^{-1}\right\}^{*}$. Equations (A.3) and (A.4) thus show that the action $\odot$ induces a right-action of the monoid $G$ over $\mathcal{N}$ that we still denote by $\odot$.

Lemma 56. For every $s \in \mathcal{N}, 1 \odot \pi_{G}(s)=s$.

This lemma can be proved by induction over the integer $n$ in the description (A.1) of a normal form $s$.

Theorem 57. The following holds:

(1) For every reduced $t$-sequence $s \in \operatorname{Red}(H, t)$, there exists $s^{\prime} \in \mathcal{N}$ with $s \sim s^{\prime}$.

(2) The set $\mathcal{N}$ is a system of representatives for the congruence $\approx$ over $H *\left\{t, t^{-1}\right\}^{*}$.

Proof. Let us first prove (1). Let $s$ be some reduced sequence given by (15). Let us show by induction over $n$ that $s$ is $\sim$-equivalent to some normal form.

If $n=0$ in (15) then $s$ is itself a normal form. Now assume that $n=m+1$. By the induction hypothesis, there exist $h_{0}^{\prime}, \ldots, h_{m}^{\prime} \in H$ such that

$$
h_{0} t^{\alpha_{1}} h_{1} \cdots t^{\alpha_{i}} h_{i} \cdots t^{\alpha_{m}} h_{m} \sim h_{0}^{\prime} t^{\alpha_{1}} h_{1}^{\prime} \cdots t^{\alpha_{i}} h_{i}^{\prime} \cdots t^{\alpha_{m}} h_{m}^{\prime} \in \mathcal{N} .
$$


First, suppose that $\alpha_{n}=1$. Let us decompse $h_{m}^{\prime}=r_{m} a$ with $r_{m} \in R_{A}$ and $a \in A$, and set

$$
s^{\prime}=h_{0}^{\prime} t^{\alpha_{1}} h_{1}^{\prime} \cdots t^{\alpha_{i}} h_{i}^{\prime} \cdots t^{\alpha_{m}} r_{m} t^{\alpha_{n}}\left(\varphi(a) \cdot h_{n}\right) .
$$

We have $s \sim s^{\prime} \in \mathcal{N}$ (note that $t^{\alpha_{n}}=t \sim a t \varphi(a)^{-1}$ ).

If $\alpha_{n}=-1$, then we decompose $h_{m}^{\prime}=r_{m} b$ with $r_{m} \in R_{B}$ and $b \in B$, and we set

$$
s^{\prime}=h_{0}^{\prime} t^{\alpha_{1}} h_{1}^{\prime} \cdots t^{\alpha_{i}} h_{i}^{\prime} \cdots t^{\alpha_{m}} r_{m} t^{\alpha_{n}}\left(\varphi^{-1}(b) \cdot h_{n}\right) .
$$

Again, we have $s \sim s^{\prime} \in \mathcal{N}$.

For point (2) in the theorem let us first show that every sequence $s$ is $\approx-$ equivalent to some normal form. As we already saw in Section 3 , for every $t$-sequence $s$ there exists $s^{\prime} \in \operatorname{Red}(H, t)$ such that $s \approx s^{\prime}$. By point (1) there exists $s^{\prime \prime} \in \mathcal{N}$ with $s^{\prime \prime} \sim s^{\prime \prime}$. Since $\sim \subseteq \approx$, we have $s \approx s^{\prime \prime} \in \mathcal{N}$.

It remains to show that two different normal forms are not $\approx$-equivalent. Let $s, s^{\prime} \in \mathcal{N}$ and $s \neq s^{\prime}$. By Lemma 56 ,

$$
1 \odot \pi_{G}(s)=s, \quad 1 \odot \pi_{G}\left(s^{\prime}\right)=s^{\prime} .
$$

Hence, $\pi_{G}(s) \neq \pi_{G}\left(s^{\prime}\right)$, i.e., $s \not \approx s^{\prime}$.

We are ready now to give the announced proof of Lemma 5. Suppose that $s_{1}$ and $s_{2}$ are reduced $t$-sequences such that $s_{1} \approx s_{1}$. By point (1) of Theorem 57 , there exist $s_{1}^{\prime}, s_{2}^{\prime} \in \mathcal{N}$ such that $s_{i} \sim s_{i}^{\prime}$ (for $i \in\{1,2\}$ ). Since $s_{1}^{\prime} \approx s_{2}^{\prime}$, by point (2) of Theorem 57 we have $s_{1}^{\prime}=s_{2}^{\prime}$. Finally, $s_{1} \sim s_{1}^{\prime}=s_{2}^{\prime} \sim s_{2}$, which proves that $s_{1} \sim s_{2}$.

\section{Appendix B. Examples of monoids}

We give here some examples of monoids $\mathbb{M}$ such that $\operatorname{Rat}(\mathbb{M})$ is closed under intersection but not closed under complement.

Example 58 (Die05). Let $\mathbb{M}=X^{*}$ be the free monoid over some infinite alphabet $X$. Since every rational expression uses only a finite number of symbols, every rational set of $\mathbb{M}$ is included in some finitely generated submonoid of $\mathbb{M}$. Hence, for every $R \in \operatorname{Rat}(\mathbb{M}), X^{*} \backslash R \notin \operatorname{Rat}(\mathbb{M})$.

On the other hand, if $R$ and $R^{\prime}$ are rational sets of $\mathbb{M}$, they are rational subsets of some free submonoid $Y^{*}$ generated by a finite subalphabet $Y \subseteq X$. By Kleene's theorem about rational sets of a finitely generated free monoid, $R \cap R^{\prime} \in \operatorname{Rat}\left(Y^{*}\right)$, and hence, $R \cap R^{\prime} \in \operatorname{Rat}(\mathbb{M})$.

Example 59. Using the same arguments as in Example 58 one can show that also for the free group $\mathbb{G}=F(X)$, generated by an infinite set $X$, Rat $(\mathbb{G})$ is closed under intersection but Rat $(\mathbb{G})$ is not closed under complement. One has to use the fact that for a finitely generated free group, the set of rational subsets is a boolean algebra [Ben69]. 
Let us construct now examples of finitely generated monoids.

Example 60. Let $X=\{a, b, c, d, e\}$. Let $L \subseteq\{b, c\}^{*}$ be some context-free language with non context-free complement. Such examples are abundant, for example one could take

$$
L=\left\{b^{p} c b^{q} c b^{r} \mid p, q, r \in \mathbb{N}, p \neq q \vee q \neq r\right\} .
$$

We then define a semi-Thue system $\mathrm{S}$ and a monoid $\mathbb{M}$ by:

$$
\mathrm{S}=a L d \times\{e\}, \quad \mathbb{M}=X^{*} / \leftrightarrow_{\mathrm{S}}^{*}
$$

Lemma 61. The set $\operatorname{Rat}(\mathbb{M})$ is closed under intersection.

Proof. Let us denote by $\pi: X^{*} \rightarrow \mathbb{M}$ the canonical projection. Let $R, R^{\prime} \in \operatorname{Rat}(\mathbb{M})$. There exist $K, K^{\prime} \in \operatorname{Rat}\left(X^{*}\right)$ such that

$$
R=\pi(K) \text { and } R^{\prime}=\pi\left(K^{\prime}\right) .
$$

Since the system $\mathrm{S}$ has no overlapping left-hand sides nor two rules with the same left-hand side but different right-hand sides, the relation $\rightarrow_{\mathrm{S}}^{*}$ is confluent (see our recalls in Section 2). It follows that

$$
R \cap R^{\prime}=\pi\left(K \rightarrow_{\mathrm{S}}^{*} \cap K^{\prime} \rightarrow_{\mathrm{S}}^{*}\right) .
$$

Since the semi-Thue system $\mathrm{S}$ is monadic, both sets of descendants $K \rightarrow{ }_{\mathrm{S}}^{*}$ and $K^{\prime} \rightarrow{ }_{\mathrm{S}}^{*}$ are rational subsets of $X^{*}$ [BJW82,Sén94]. Equality (B.1) thus shows that $R \cap R^{\prime}$ is a rational subset of $\mathbb{M}$.

Lemma 62. For every $R \in \operatorname{Rat}(\mathbb{M})$, the language $\pi^{-1}(R)$ is context-free.

Proof. Let us assume that $R=\pi(K)$ for some $K \in \operatorname{Rat}\left(X^{*}\right)$. By the confluence property of $\rightarrow \mathrm{s}$,

$$
\pi^{-1}(R)=\left\{u \in X^{*} \mid \exists v \in K \rightarrow_{\mathrm{S}}^{*}: u \rightarrow \rightarrow_{\mathrm{S}}^{*} v\right\} .
$$

We already observed that $K \rightarrow \mathrm{S}_{\mathrm{S}}^{*}$ is rational. Since the set $a L d$ is context-free, for every rational set $Q, Q \leftarrow \mathrm{S}_{\mathrm{S}}^{*}$ is context-free. Applying this property to $Q=K \rightarrow{ }_{\mathrm{S}}^{*}$ we get that $\pi^{-1}(R)$ is context-free.

Lemma 63. The set $\operatorname{Rat}(\mathbb{M})$ is not closed under complement.

Proof. Let $R=\{\pi(e)\}$. Clearly, $R$ is a rational subset of $\mathbb{M}$. Let $R^{\prime}=\mathbb{M} \backslash R$. One can check that

$$
\pi^{-1}\left(R^{\prime}\right) \cap a\{b, c\}^{*} d=a\left(\{b, c\}^{*} \backslash L\right) d .
$$

Since $\{b, c\}^{*} \backslash L$ is not context-free, $\pi^{-1}\left(R^{\prime}\right)$ is not context-free (by the closure of context-free languages by intersection with rational sets). By Lemma 62 we can conclude that $R^{\prime}$ is not rational. 
We don't know, whether there exists a finitely generated (or even finitely presented) group $G$ such that $\operatorname{Rat}(G)$ is closed under intersection but not closed under complement. 